\title{
Designing visualization and interaction for industrial control rooms of the future
}

\section{Veronika Domova}
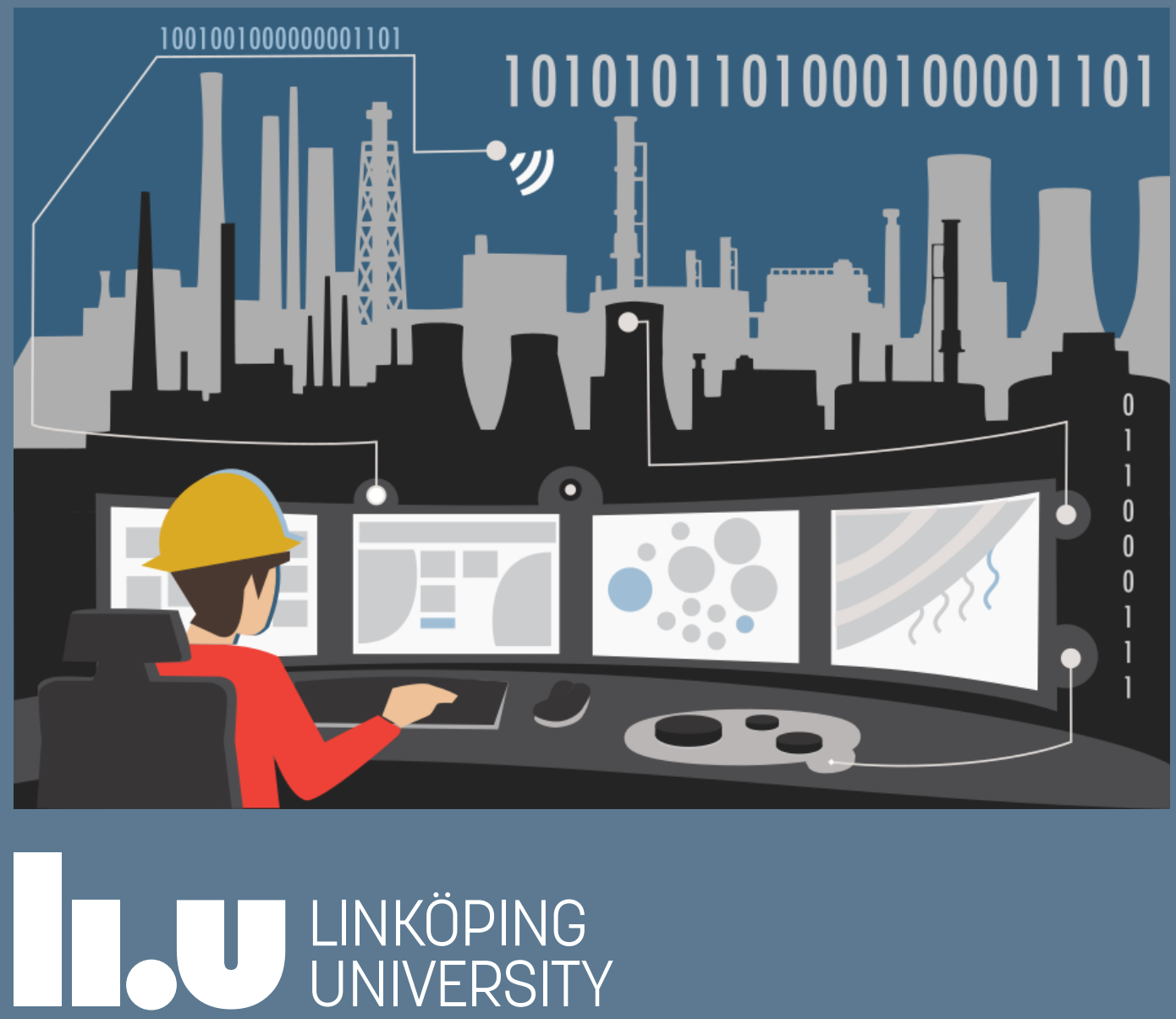

LINKÖPING UNIVERSITY 


\title{
Designing visualization and interaction for industrial control rooms of the future
}

\section{Veronika Domova}

\author{
Linköping University \\ Faculty of Science and Engineering \\ Division of Media and Information Technology \\ SE-601 74 Norrköping, Sweden
}

Linköping 2020 
(C) Veronika Domova, 2020

ISBN 978-91-7929-835-7

ISSN 0345-7524

Printed by LiU-Tryck, Linköping 2020 


\section{POPULÄRVETENSKAPLIG SAMMANFATTNING}

Under de senaste årtiondena har industrin digitaliserats i stor utsträckning och består nu av komplexa strukturer där människor, maskiner, autonoma agenter och sensorer samspelar. Människor arbetar ofta som operatörer i industrin, med uppgift att övervaka och styra maskinella processer på distans från kontrollrum. Den omfattande digitaliseringen medför nya utmaningar för operatörerna i och med att mängden tillgänglig information växer samtidigt som kontrollrummens användargränssnitt är otillräckliga för de ändrade förutsättningarna. I den här avhandlingen utforskar jag nya former av visualisering och interaktion som skulle kunna hjälpa operatörerna hantera de växande informationsmängderna och sköta sitt arbete på ett effektivt, tillförlitligt och säkert sätt.

Jag presenterar flera exempel på skräddarsydda datavisualiseringar som minskar belastningen på operatörerna genom att kombinera stora mängder data i kompakta, icke-triviala presentationsformer. När det gäller interaktion föreslår jag flera fysiska och taktila interaktionsformer som kan göra operatörernas arbete mera fritt och självstyrt. Jag presenterar slutligen koncept för adaptiva system som anpassar sig till operatörernas arbetssituation för att skapa smidig interaktion och stödja hög situationsmedvetenhet.

Avhandlingen vänder sig i första hand till interaktionsdesignområdet, men jag räknar med att den kan vara av intresse för en bredare publik i och med att den behandlar användarupplevelse. Vi använder alla tekniska produkter och tjänster i någon utsträckning och bör därför kunna relatera till industrioperatörernas utmaningar. Mitt arbete ger också en inblick i hur människans arbetssituation ser ut i dagens industri. 


\begin{abstract}
During the last decades, the industry has been undergoing extensive digitization leading to complex ensembles of humans, machines, autonomous agents, and sensors. With this new setup comes the challenge of how to appropriately support work-practices of industrial operators who now need to monitor and control complex industrial processes through digital interfaces. Information overflow and restrictive interfaces are two significant problems that operators face in their daily routines. In this $\mathrm{PhD}$, I explore approaches to visualization and interaction that would reduce industrial operators' information load and enable them to perform their duties in an efficient, reliable, and safe manner. Industrial users and industrial settings are the starting points of my research.
\end{abstract}

In this thesis, I describe multiple examples of custom-tailored data visualizations that reduce the operator's visual load by consolidating large amounts of data into compact overview displays with often nontrivial data presentation. With respect to interaction, I propose several tangible and tactile interfaces, as well as concepts for natural interaction, that let the user freely interact with the control station and the information it depicts. Finally, I propose several concepts of adaptive systems that adjust to the operator's context to ensure their high situational awareness and convenience of interaction.

Even though this thesis is primarily intended for the community of interaction designers, I expect it to be of interest to a broader audience due to its relation to the user experience field. To a certain extent, everyone can resonate with the user's problems because, in our everyday life, we all are users of some technology and services. Furthermore, for a lay reader, this work can be seen as a comprehensive introduction to how the industry works and what role the human plays there. 


\section{Acknowledgments}

First of all, I would like to express my sincere gratitude to my academic supervisor Professor Jonas Löwgren. His continuous support, insightful comments, patience, and immense knowledge helped me to achieve productive results and reach a new level in my academic career. Also, I would like to thank my industrial supervisor Elina Vartiainen who inspired me to start a $\mathrm{PhD}$ and provided guidance throughout the process. Most importantly, I would like to thank my partner Amir Masoumi who was supporting me in this long journey by all possible means, passionately believed in my work, truly shared my victories and failures; I could not have imagined having better support. Moreover, I would like to thank the Wallenberg Foundation for sponsoring my research, as well as for providing me the opportunity of spending a short sabbatical at Stanford. That trip was a truly fantastic and eye-opening journey which I will never forget. Moreover, I would like to thank my colleagues from ABB Corporate research, as well as from RISE, who joined me on different stages of this long journey. Finally, I cannot forget to thank my family and friends who had enough understanding and patience to accept my busy schedule. 



\section{Contents}

Abstract iii

\begin{tabular}{lll}
\hline Acknowledgments & v
\end{tabular}

Contents vii

\begin{tabular}{lll}
\hline Introduction & 1
\end{tabular}

$1.1 \quad$ Industrial process control . . . . . . . . . . . . . . . . 1

1.2 Problem statement $\ldots \ldots \ldots \ldots$. . . . . . . . . . . . . . . . . .

1.3 Research questions . . . . . . . . . . . . . . . . . . . . . 13

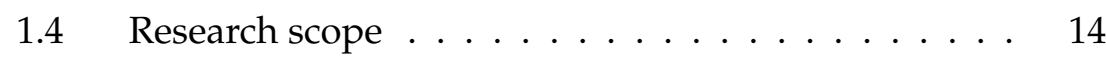

1.5 Papers included in the thesis . . . . . . . . . . . 17

1.6 The complete list of my academic publications . . . . 22

2 Theoretical framework 25

$2.1 \quad$ Levels of Automation . . . . . . . . . . . . . 26

2.2 Situation awareness . . . . . . . . . . . . . . . . . . . . 29

2.3 Human perception and attention . . . . . . . . . . 32

2.4 Human performance. . . . . . . . . . . . . . . . . . . . . . . . 35

2.5 Information visualization . . . . . . . . . . . . . 38

$2.6 \quad$ Interactive spaces . . . . . . . . . . . . . . 42

\begin{tabular}{lll}
\hline 3 & Method & 47
\end{tabular}

$3.1 \quad$ User-Centered Design . . . . . . . . . . . . . . . . . . . . . . . 48

$3.2 \quad$ Research through Design . . . . . . . . . . 50 
3.3 Research process at ABB Corporate Research. . . . . 53

$3.4 \quad$ My research practices . . . . . . . . . . 57

$\begin{array}{lll}4 & \text { Conducted work } & 65\end{array}$

$4.1 \quad$ Data visualization . . . . . . . . . . . . . . 70

$4.2 \quad$ Interactive spaces . . . . . . . . . . . . . 81

\begin{tabular}{lll}
\hline 5 & Results & 87
\end{tabular}

5.1 RQ1: How to support the operator's situation awareness under information overload. . . . . . . . . . . 87

5.2 RQ2: How to facilitate the operator's interaction with the operator workstation. . . . . . . . . . . . . . 92

5.3 RQ3: How to reduce the information overload of the operator. . . . . . . . . . . . . . . . . 95

$5.4 \quad$ RQ4: How to bridge the gap between physical industrial processes and their digital representation. . . . . 98

$\begin{array}{lll}6 & \text { Discussion } & 101\end{array}$

$6.1 \quad$ Methodology . . . . . . . . . . . . . . . . . 101

6.2 Future control rooms . . . . . . . . . . . . . . . . . 111

\begin{tabular}{lll}
\hline 7 & In conclusion & 117
\end{tabular}

\begin{tabular}{ll}
\hline Bibliography & 121
\end{tabular} 


\section{Chapter 1}

\section{Introduction}

\subsection{Industrial process control}

For millennia, production was essentially manual with the human at the center of the process. It was taking place in small family workshops where the human was in close contact with materials, tools, and the final product, and had total control of the entire process. As the human was working with one product at a time, the cognitive load was tolerable. The status of the production process was perceived through sensory inputs, i.e. sounds, smells, physical properties, such as weight, color, etc. Such production setup assumed low productivity and low output [160]. Production volume increased as, with the flow of time, more sophisticated tools, machines, and approaches were appearing. Industrial revolutions brought especially drastic changes in this development. Factories appeared during the First Industrial revolution, i.e. in the late 18th early 19th centuries, and continued their rise under the Second Industrial Revolution, i.e. in the late 19th and early 20th centuries [35, 160]. During the first half of the 20th century, a typical shop-floor operator was stationed at one machine performing standardized, monotonous tasks with low complexity; advanced knowledge was not required as decisions were taken by higher-level 
managers [108]. With the continuous flow of technical innovations, it became possible to automate some manual tasks and perform process control from panels located close to or placed on the process equipment. Such a setup required personnel to be constantly present at the machines, see Figure 1.1 .

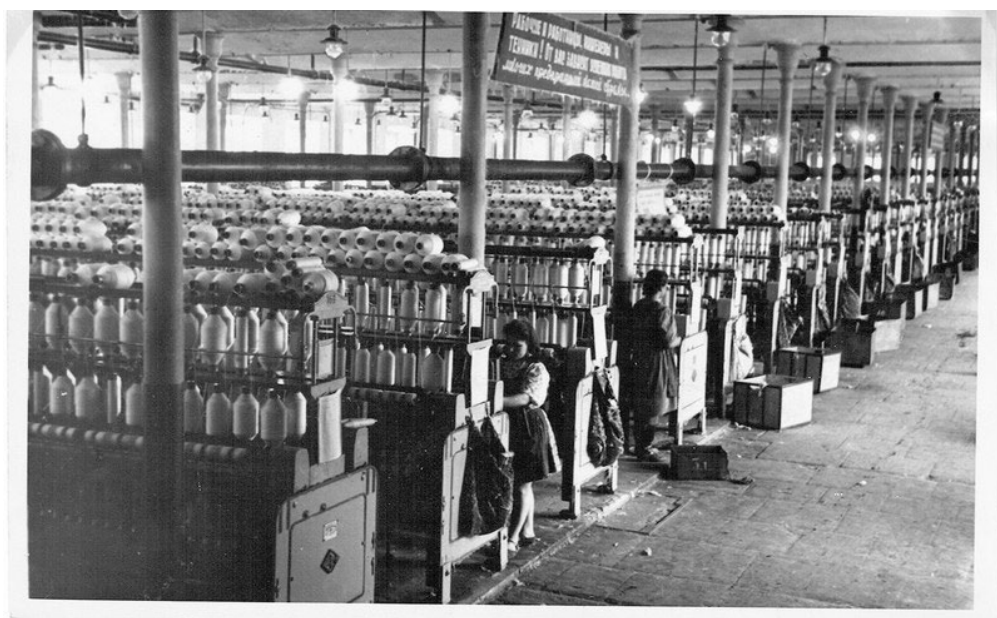

Figure 1.1: Cotton-spinning machines at the Krenholm Manufacturing Company, approx. year 1950-1957. Source: Narva Muuseum, photo number NLM KMM F 17:4, http:/ /www.muis.ee/museaalview/2513110

The next significant development step undertaken in 1950s comprised the centralization of all the localized control panels in a permanentlymanned central control room placed in a close proximity to the actual production, see Figure 1.2. The operators interacted with the production process through single-sensor-single-indicator panels with dials, gauges, buttons, lamps, etc. Information was presented statically all at once, in a parallel form. Therefore, to be able to monitor the production process, operators had to know where all of the different indicators were physically located [231]. With the centralization came the advantage of reduced manpower requirements and the possibility appeared to get a comprehensive overview of the entire production process. Centralized control panels had certain drawbacks. For exam- 


\subsection{Industrial process control}

ple, it was very hard to upgrade such interfaces, e.g. introduce new interaction controls into an existing control panel. Furthermore, due to the linear layout of panels, the operators had to physically move around the control room to be able to maintain high situational awareness of what is happening in the plant. Vicente et al. [231] point out that such a layout made it difficult for operators to communicate with each other, therefore, for example, it was not trivial to understand when another operator needed help. Similarly, it was very difficult for operators to remain aware of the state of a unit after having physically left it. Moreover, analog hard-wired control panels were fixed in their location and grouping, therefore they did not allow any degree of freedom for tailoring the presented information to a particular task at hand. To overcome this issue, operators had to go outside of the means explicitly provided by the design using ad hoc methods such as creating external reminders [231].

The Third Industrial Revolution, which started in the 1970s, brought automatic production based on electronics. With the arrival of CPUs, networks and graphical computer displays, it became possible to replace analogous control panels with digital interfaces and form stationary workplaces. The digitalization significantly changed the lookand-feel of the industrial control rooms, as well as the workflows of operators [231]. Digital interfaces offered a wealth of flexibility in terms of how information could be depicted on the screens which allowed the operators to adjust the information presentation to their current task [231]. With the transition to digital control systems, many authors [234, 97, 231, 120, 132] agreed that the graphical user interface (GUI) of the digital control system became one of the most critical factors for improving operators' efficiency and preventing safetyrelated issues. Han et al. [97] write that early GUIs were prone to a common mistake when a man-machine interface (MMI) design was directly transformed into a GUI design by miniaturizing and condensing MMI controls on a computer display which diminished the advantage of using a GUI. Later, various guidelines for user interface design 


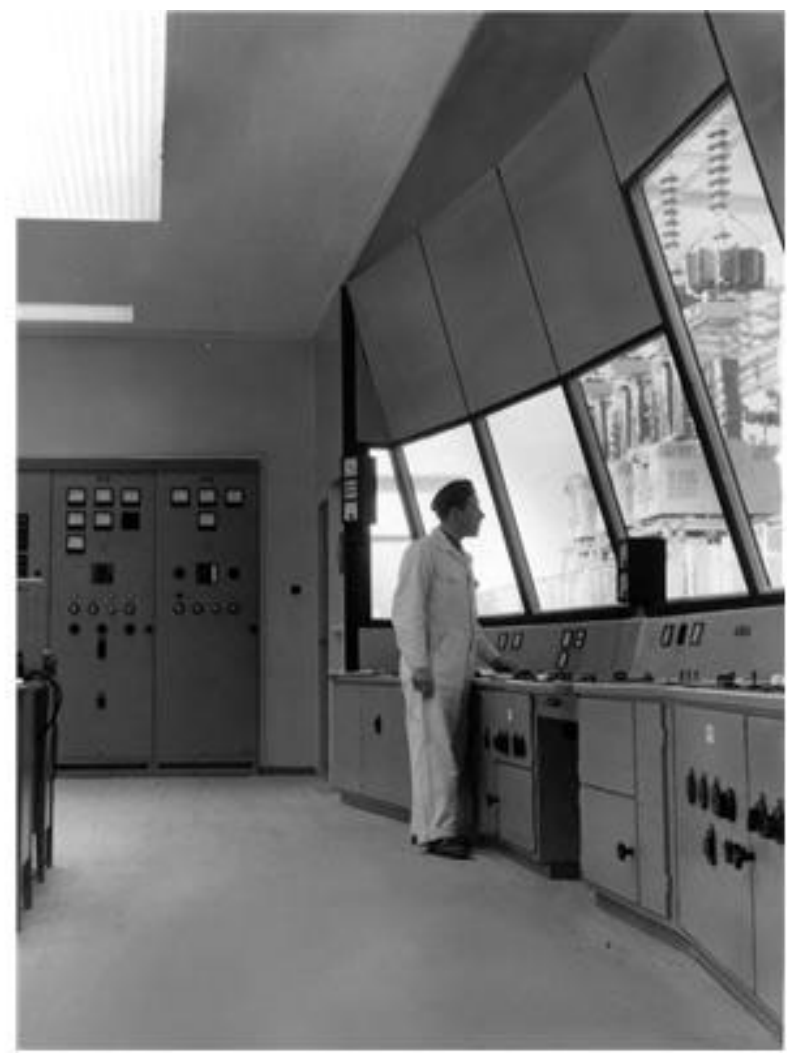

Figure 1.2: A control room at the Gotland HVDC link built in 1954. The image is taken from ABB internal archive.

were proposed consisting of high- and middle-level theories, models and principles, design standards, practical recommendations, and evaluation strategies. Operators, however, were finding the transition to digital interfaces challenging because they felt that digital information alone was not enough to support their need for understanding the real status of the control process [25]. The requirements for the industrial operator have also significantly changed. If earlier the operator was more or less focusing on one particular machine or process conducting repetitive tasks, the operators of digital control rooms started facing increased responsibilities in a wide-ranging spectrum of tasks starting from manual maintenance skills to data analytics. Operators 


\subsection{Industrial process control}

were now expected to behave proactively, i.e. predict the system's behavior and forecast occurring problems. As a result, from now on, operators could be identified more as knowledge workers with technical skills rather than merely technicians [1].

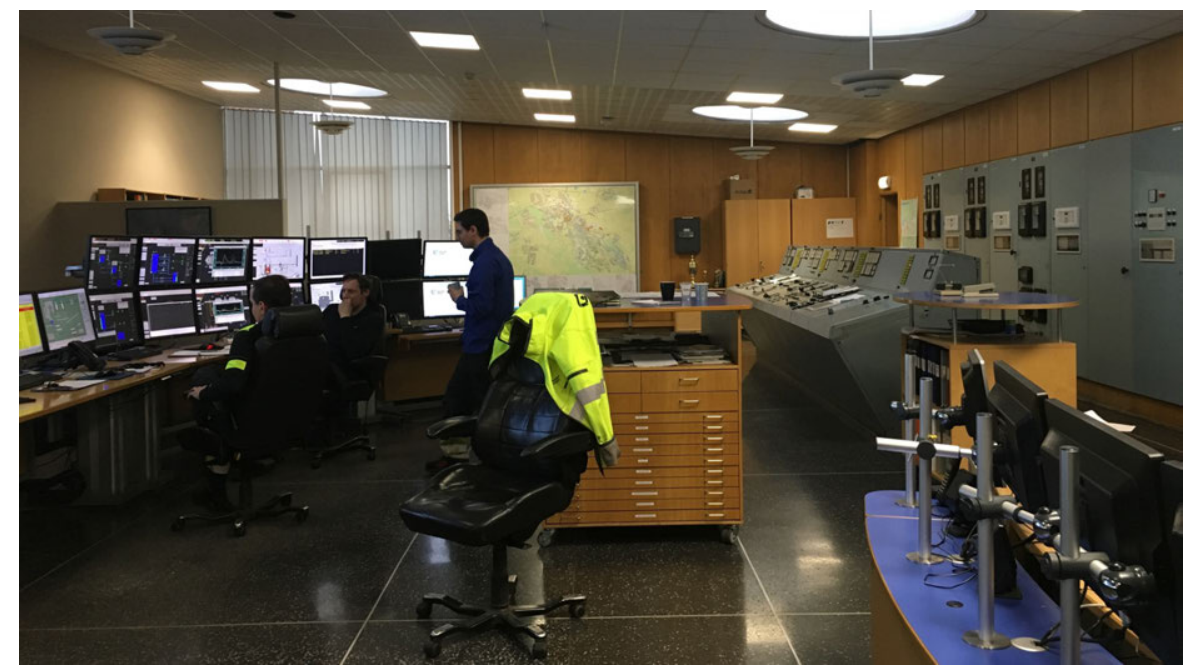

Figure 1.3: A control room at one of the visited industrial sites. The control station consists of more than 17 screens that serve for different purposes. On the right, there is a setup of legacy control panels that are occasionally still in use.

Since the transition to digital control rooms in the 1970s, the physical setup of industrial control rooms has not significantly changed. They are office spaces where operators are monitoring the status of the production processes. Importantly, the modernization of control rooms can significantly vary from one another. Some control rooms that I have visited are rather outdated, with old-fashioned monitors of different sizes stationed on simplistic desks and keyboards and mice chaotically spread around the tables, some of them still have control panels in use, see Figure 1.3 . Others are fully modernized with the latest holistic motorized control stations that can be ergonomically adjusted to the operator's needs. All in all, regardless of the modernity of the interfaces and look-and-feel of the rooms, the work processes in the modern industrial control rooms are very similar. Most of the 
time the operators spend in control rooms only occasionally visiting the field. An operator's workplace is a workstation comprising an office table with a dozen screens, several mice, and keyboards. The operators monitor and control industrial processes via the displays of the control station, see Figure 1.3. The information on the displays is mainly visualized using schematic data presentations, e.g. process and instrumentation diagrams (P\&IDs). A P\&IDs, see Figure 1.4, is a detailed diagram used for laying out the piping and process equipment of an industrial process together with the instrumentation and control devices [166, 115]. Dashboards with numerical KPIs (Key Performance Indicators), tables, and real-time videos from the field are other typical examples of information visualization in modern industrial control rooms.

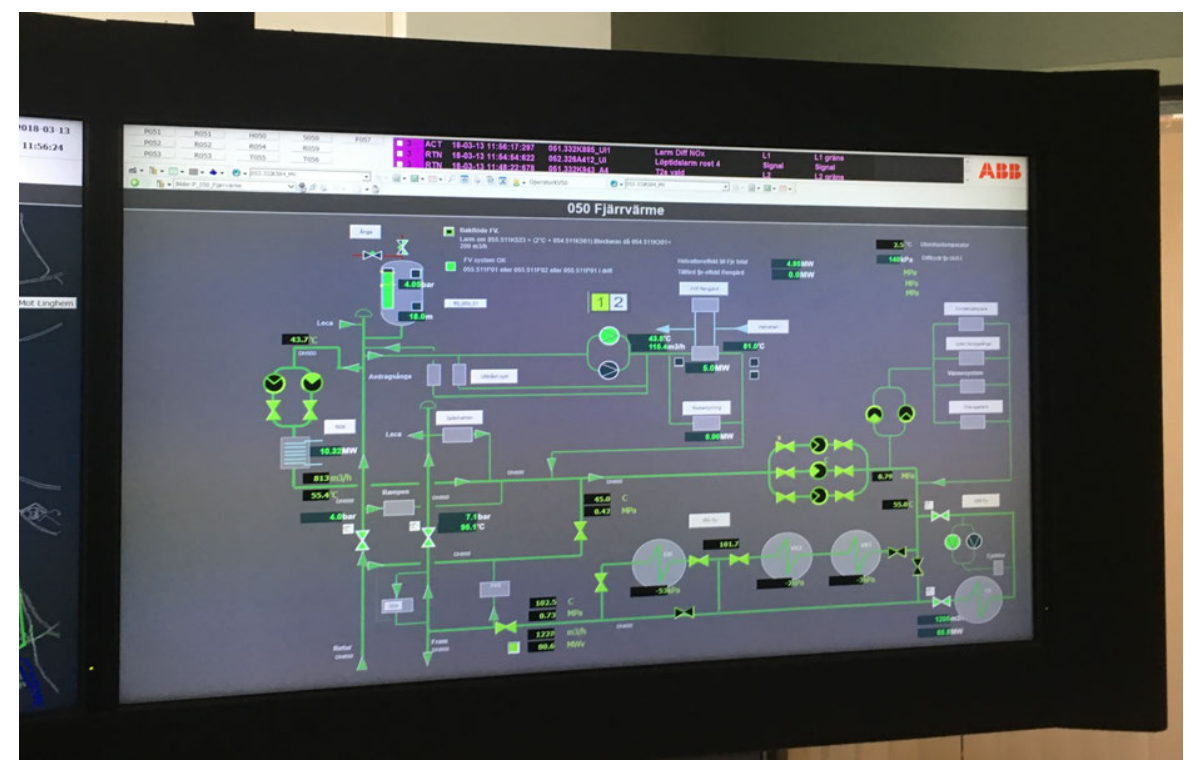

Figure 1.4: An example of a PID diagram in the control interface at one of the visited industrial sites.

Operators are getting notified about extraordinary events through alarm systems. Alarms and warning signals are indicators presented in visual, i.e. through colorful notifications on displays, audio, and sometimes light forms to inform the operators about extraordinary 
conditions. An alarm system is a combination of hardware and software that deliver alarms to operators. Alarm systems are central elements in the operation of production as they continuously monitor the production and notify operators about undesired process states that require their professional judgment or action. Routine alarm management incorporates noticing an alarm once it is activated and taking the necessary corrective actions to mitigate its source [111].

Industrial operators work in shifts. Shift handover, taking place when a new group of operators comes in to take over, is an important mechanism for the new coming operators to obtain situation awareness. For this purpose, operators have brief meetings with the previous shift and take system printouts to obtain a more sufficient understanding of the current state of the processes, devoting an extra effort if needed, e.g. going through system logs [231].

\subsection{Problem statement}

During the last decades, the process of extensive industrial digitalization, which is often referenced to as the Fourth Industrial Revolution, i.e. Industry 4.0, has been gaining pace. Industry 4.0 features the convergence of physical and digital worlds by means of sensors, actuators, algorithms, and networking. The resulting cyber-physical production systems (CPPS) strive to make the production more costand time-efficient, as well as safe. The extensive digitalization brought multiple challenges into industrial control rooms. First of all, the legacy interfaces prevalent in industrial control rooms, i.e. industrial control stations with process control graphics on the interfaces, as well as alarm systems, were designed during the times when the data volumes were rather modest. The most typical response to the increasing data flow is to expand these interfaces by adding more screens, introducing more system views and making them more detailed. As a result, screens of different sizes and purposes are flooding indus- 
trial control rooms, see Figure 1.5. For example, Vicente et al. report that, during their field studies in control rooms of several modernized power plants, they observed "many different display screens, many more than can possibly be viewed at any one time" [231].

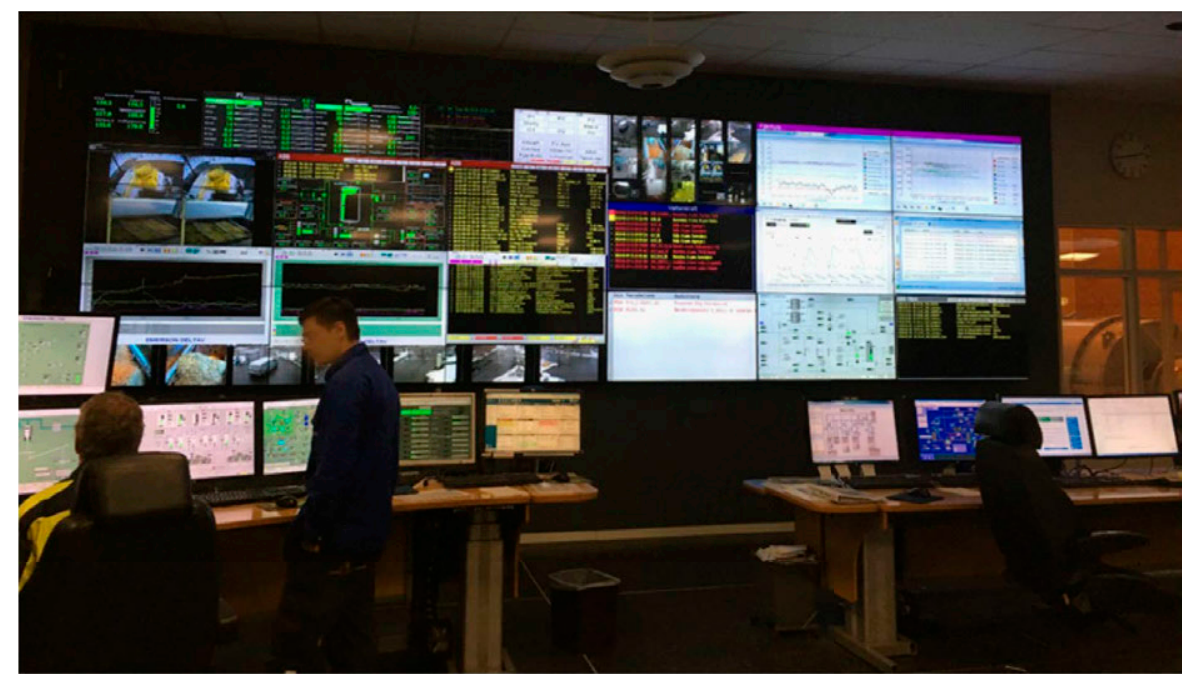

Figure 1.5: A multitude of screens in a control room at one of the visited industrial sites.

Importantly, as the dataflow grows exponentially in both size and complexity, human cognitive capacity, such as attention and shortterm memory, remains essentially the same, thus becoming the limited resource [137]. No surprise that operators become cognitively overloaded with too much visual information and too many displays. Ironically, people generally enjoy seeking for information and feel satisfied when new information is found [18]. Such heuristic work provides intrinsic motivation through a sense of accomplishment [181]. Furthermore, people tend to want more information and more choices than they can process [117]. There is some evidence that too much information and too many choices will prevent people from choosing at all, i.e. the information overload actually paralyzes thinking [118]. The information overload problem describes the danger of the human getting lost in data, which is either not related to their task at hand 
or is presented in an inappropriate way [133]. This visual and cognitive overload can lead to difficulties in prioritization, reading and comprehending information, and reduce the ability to make the right decisions [38]. Drowning in a multitude of screens, the operator might occasionally not attend to the one showing critical information risking to miss essential data, e.g. alarms. In the scope of a broad evaluation of alarm system interfaces, Han et al. [97] found that even when warning signals were presented on the interface, many of them could not catch the operators' attention to be easily perceived. With respect to the acceptable alarm delivery rate, several industrial standards [114, 66] recommend to limit the alarm flow to no more than 6 per hour per each operator. In reality, most alarm systems are poorly configured and cannot meet these requirements overloading operators with nuisance alarms that distract their attention from real problems [111]. Furthermore, due to the increased alarm flow, operators often switch off the sound notifications to avoid the constant noise, therefore, they might not recognize presence of alarms unless they fix their eyes on the appropriate screen.

Furthermore, as the amount of system views grows faster than the number of screens, not all the views can be shown simultaneously to the user, but only a limited subset of them. This, in turn, creates the socalled keyhole effect [239], i.e. the reduced visibility when the size of the virtual information space is much larger than the viewport available to the user. As a consequence, operators might miss important information or get only a limited understanding of the ongoing situation in production. Navigation among system views is another source of trouble. Operators should be aware of how to bring up the information they want to be shown on the screens which increases the amount of knowledge required to operate a digital control system [231]. Due to the increasing complexity of industrial systems, navigation can get overly complicated. For example, Vicente et al. [231] report a menudriven interface that has a hierarchical structure with approximately 45 system views at the highest level of the hierarchy; Han et al. [97] 
report more than 100 views of the control system at a steel manufacturing factory. For this reason, simple operations, such as locating a system view containing an object of interest, are getting much more difficult. The complexity of the interfaces and many degrees of freedom they provide put extra pressure on the operators who have to spend a significant amount of time manipulating the interfaces rather than monitoring the production. Furthermore, design of control interfaces is prone to many usability issues. For example, the case study in a control room of a steel manufacturing factory by Han et al. [97] identified more than 500 usability issues, such as a) too many system views not directly relevant to the operator's tasks, b) redundant information, and c) not salient enough important information in the system views. Vicente et al. [231] point out that usability problems of control room interfaces "make monitoring more difficult, not because the job itself is more difficult, but because the job has to be done with an interface that does not support monitoring activities as well as it could".

Another significant problem of the industrial control room is that operators are too tethered to their control stations. The information presented by the control station is only comprehensible when the operator is in close proximity to the screens. The user interface (UI) elements are simply too small to be distinguished from a distance of several meters. Furthermore, interaction with the control station goes only through keyboards and mice that also require physical proximity. All in all, the operator's situation awareness is dependent on the proximity to the control station. Every time they move even a couple of meters away from the control station they risk losing their situational awareness and understanding of the ongoing situation, not to mention the ability to control the processes. Sadly, during field studies, I have witnessed industrial operators eating their lunch right at the desk or thinking twice before going to the toilet.

Moreover, after plant control was moved to centralized control rooms, the operators became disconnected from the actual production [251]. On one hand, being isolated from the field is beneficial as industrial 
production is often noisy, dirty, and dangerous. On the other hand, the physicality of the industrial environment is inseparable from the activities that are going on there, including field workers' workflows; operators receive additional knowledge about the status of the production process through tactile sensations, sounds, and visual observation. Even though, due to the extensive digitalization, the nature of the knowledge required from the operators is changing, the skills in the industry are still learned in a situated way [151, 1], i.e. acquired by actually engaging in physical interactions in a real-world industrial context. As such, even newly educated operators are familiar with the physical properties of the equipment they are in control of. However, this knowledge is not captured in the interfaces nor facilitated in the input and output devices used in modern industrial control rooms. When working with GUIs, operators cannot take advantage of their technical proficiency learned in situ nor utilize their skills for manipulating physical objects. Furthermore, physical properties and effects of the industrial environment and machinery can be a source of information for humans. Therefore, some feel that GUIs act to further separate operators from industrial environments. For example, Müller et al. [164] outline that conventional visual interfaces widely used in industrial control rooms lack process-related interactivity such as haptic feedback, physical constraints, and the involvement of motor skills. Heyer and Husoy [105] cite operators of an oil and gas facility who expressed that "feeling" the plant is important for maintaining situational awareness, diagnosing faults, and determining what corrective action to take.

The last but not least important problem is related to the prevalence of legacy interfaces in industrial control rooms. As the control stations grow more complex, keyboards and mice become in many ways restrictive. For example, an average control station that comprises 9 monitors, would require 3 or 4 keyboards and mice respectively. One can imagine that looking for the right mouse to control an interface in focus can take a moment. To add more, operators often complain 
about mice and keyboards getting dirty, mostly because operators tend to get their hands dirty when going to the field, which repels from touching them. Another problem is that in addition to not keeping pace with the tempo of industrial digitalization, they also repel younger generations of industrial operators from joining the industry. The so-called Net generation [220], i.e. people under the age of 30 who grow up with the Internet, interactive technologies, social networks, and online access to information, have different habits in social interaction and approaches to problem-solving. As a result, they have certain expectations related to technology they are working with on a daily basis. Obviously, they are not willing to deal with outdated operating systems and boring, over-complicated interfaces. With respect to the industry, the gap between the interaction technologies used in consumer products, especially in the gaming industry, and those available in industrial control rooms is already too large and continues to expand. No surprise that many industry representatives are talking about the problem of recruiting young people [108]. In the interviews conducted by Holm et al. [108] with production and HR managers at several Swedish manufacturing companies, a few interviewees expressed a hope that an increased level of interactive technology in the industry will help to recruit young people in the future.

In this chapter, I listed only a fraction of known problems encountered in industrial control rooms in connection to the extensive digitalization and automation of the industry. All in all, the underlying message is that the existing tools simply cannot effectively accommodate new and emerging conditions, such as growing amounts of data. Therefore, it is only natural to admit the need for a new set of interfaces that would support work-practices of industrial operators under the changing work conditions [92]. 


\subsection{Research questions}

The problems of industrial control rooms listed in the previous section underpin my PhD research. The overall goal of my research is to improve user experience in industrial control rooms and turn industrial control rooms into more user-oriented environments with interfaces that assist rather than stress. With this goal in mind, I have outlined four major research questions to be addressed in the scope of my $\mathrm{PhD}$ research.

RQ1: How to support the operator's situation awareness under information overload. Having too many screens and too many system views, as well as a constant flow of alarm notifications, the operator can never be sure that they are attending to the most urgent information at the moment and have the right understanding of the situation in the production, i.e. up-to-date situation awareness. Perhaps, the right way to deal with this problem would be to reduce the very amount of information delivered to industrial control rooms, but that would require a holistic reconsideration of the means by which the industry is getting digitalized. Obviously, in the scope of my $\mathrm{PhD}$ research, I cannot do anything about the growing amounts of data. Instead, I would like to find ways to ensure the operators' situation awareness in the situation of information overload by means of effective data visualization, as well as through flexible interaction.

RQ2: How to facilitate the operator's interaction with the operator workstation. The operator's workstation is restrictive in many aspects starting from limiting interaction means to the required proximity. Working in such constrained conditions has a direct influence on the operator's performance and well-being at work. From my point of view, the control station, which is essentially the operator's working place for eight hours per day, should not be their prison, but rather an assistive environment with a rich spectrum of interaction possibilities. In my research, I would like to find ways of increasing the flexibility in the interaction between the operator and their control station. 
RQ3: How to reduce the information load of the operator. Next, I would like to focus on the problem of operators getting overloaded with too much information. In my eyes, the approach of blindly adding new screens and new system views is only a temporary solution in dealing with growing amounts of data. At some point, they will inevitably exhaust their potential. In my research, I would like to investigate other ways of presenting industrial information that could become an alternative to the conventional process graphics, which is usually too detailed and requires a lot of visual space. In addition, I would like to explore the potential of other interaction modalities that could potentially reduce the cognitive load when interacting with overly complicated control stations.

RQ4: How to bridge the gap between physical industrial processes and their digital representation. My last research question is dedicated to the problem of digital interfaces increasing the gap between the actual industrial processes and industrial operators. Existing digital interfaces are too pragmatic conveying only numeric and schematic data, which prevents the operators from envisioning what they are actually in control of. As a result, from the interface perspective, there is not much difference between controlling a power plant or a pizza production factory. In my research, I want to find ways of reintroducing the physicality of industrial processes into industrial interfaces, i.e. physical properties that are an integral part of production processes such as sound, smell, temperature, etc. In my eyes, this can help operators to keep a stronger bond with the field and better understand the effects of their manipulations in the control system on the actual production.

\subsection{Research scope}

In the scope of my $\mathrm{PhD}$, I focus on designing and developing novel industrial data presentation approaches and interaction means for in- 
dustrial control rooms. My goal is to assist operators in their workflows under the new circumstances of extensive industrial digitalization. In my work, I do not question ethical questions of whether the extensive digitalization and automation of industrial production is good or bad. I am aware that there are different opinions about the subject in terms of the influence on unemployment in society, consumption of resources, pollution, globalization, etc. In my eyes, regardless of whether the process will continue at the same pace, accelerate or eventually slow down, the important fact with respect to my research is that, all in all, digitalization and penetration of automation have already changed the working context of industrial operators. In particular, growing amounts of data and the complexity of digital interfaces reveal the need for supporting operators in their workflows. Therefore, the goal of this work is solely to assist industrial operators to do their jobs more effectively under the new conditions.

The work of industrial operators comprises a complex nexus of different aspects such as human factors, levels of automation, industrial process complexity, control room setup, etc. In my research, I could not possibly touch upon all the factors that influence the workflows of operators. In this section, I would like to explicitly point out some limitations of my work.

First of all, in the course of my research, I do not take into consideration the collaborative aspect of work in industrial control rooms. I am aware that collaboration is an important part of the industrial operator's workflows. Operators collaborate on solving problems in industrial control rooms, in the field, as well as with remote colleagues. Earlier in my career, I developed a spectrum of solutions [58, 228, 59] that were intended to support remote and local collaboration in industrial control rooms. However, in my $\mathrm{PhD}$, I have deliberately decided to focus on the individual work of industrial operators. This choice was motivated by the fact that, even though occasional collaboration is inevitable, operators are often on their own when monitoring the production and solving problems. 
Furthermore, in my work, I do not take into account the social effects that are normally taking place in industrial control rooms. For example, operators are usually sharing a common working space, i.e. they are not alone when performing their tasks. Moreover, under some non-routine circumstances, such as a start-up of a plant, their actions are closely observed by supervisors and managers who are eager to return to normal production rate as soon as possible [144]. Such copresence and increased attention per se can result in arousal and influence operators' actions and decisions leading to both facilitating and impairing effects. There is a body of literature [26, 5, 244] reporting changes in performance when performing a task while being observed or alone. Even though the topic of social facilitation in the industrial domain is understudied, there is some evidence that social facilitation and other factors of social stress might influence the performance of human-automation interaction [194].

Another limitation of my work is that I solely focus on the operator's routines while in control rooms. In reality, operators often shift between the field and the control room, which adds extra pressure on maintaining situation awareness and brings other issues, such as the need of carrying tools around or the problem of having dirty hands and clothes. Some aspects of my work on the interactive zones [52] indirectly address these issues, for example, the set of alternative interaction means, such as gaze, voice, and gestures, can be useful when the operator is returning from the field having dirty hands or carrying tools and generally cannot or does not want to touch keyboards and mice of the control station. All in all, the fieldwork aspect of operators' workflows was not a particular focus of this work.

The last but not least significant limitation of this work is that I do not take into account personality traits, cultural and demographic specifics, physical abilities of industrial operators. Industrial operators are first and foremost humans, therefore, they are very diverse, e.g. old, young, women, men, experienced, inexperienced, introverts, extroverts, shy, active, impaired, etc. Some of these personal aspects 
can have a very strong effect on the spectrum of suitable interfaces for a particular user. For example, physical disabilities might make some interfaces or interaction means completely unacceptable. Take, for instance, color blindness. Nine percent of men and one-half percent of women are color-blind, i.e. they have a color deficiency that prevents seeing differences between some colors, most commonly between reds, yellows, and greens [236]. As a takeaway, ideally, when designing interfaces, one should employ colors that work for everyone, for example, various shades of brown. Culture is another factor that can influence technology acceptance, as well as operators' behavior in different circumstances. All in all, I did not consider any of these aspects in my work.

Finally, I would like to add that some of the limitations described in this chapter were of necessity, mostly due to the time limitations of my research projects, while others were deliberate to reduce the scope of the work. In any case, I want to express the hope that these limitations will not diminish the results of my work in the eyes of the reader. Also, I believe that some of these limitations can inspire others to open up new directions for future research.

\subsection{Papers included in the thesis}

Veronika Domova. "Fidgeting with the Environment: a Tangible Control for Interacting with a Smart Light". Submitted. [50]

In this work, I explored the potential of considering the user's behavior in a particular environment when designing a control interface for controlling technology in this environment. As an embodiment of my ideas, I designed and developed one example of such an interface, i.e. a hand-held tangible artifact with fidgeting features intended for interaction with a smart light in a home office environment. I worked solely on the concept idea, implementation, and on the paper. 
Veronika Domova. “Guiding the Operator's Attention Among a Plurality of Operator Workstation Screens". Submitted. [51]

In this work, I explored the possibilities of visual aid systems to help guide operators' attention in the multitude of control station screens. For this purpose, I developed and evaluated two visual aid systems. I was the main leader in this work with respect to the ideation, design, and implementation, however, I engaged a master thesis student who was helping me with the development of the high-fidelity prototypes and arrangement of the evaluation tests. I worked alone on writing the paper.

Veronika Domova, Erik Gärtner, Johan Källström, Martin Pallin, Fredrik Präntare, and Nikita Korzhitskii. "Improving Usability of Decision Support Systems for SAR Operations: WARA-PS case study". Submitted. [54]

This is a collaborative work between me and my colleagues from the WASP program. It describes our workflow when improving the usability of the interface of one existing search and rescue control system. In the project, all the participants took the lead of project-related tasks belonging to their expertise. My role was to design and develop the dashboard which was one of the extensions of the system. I did not participate in the field studies and the evaluation of the final solution due to being on sabbatical abroad, however, I was an active participant in the subsequent analysis phases. Also, I took the leading role in writing the paper.

Veronika Domova and Katerina Vrotsou. "A Model for Types and Levels of Automation in Visual Analytics: Examples from EventSequence Analytics". Manuscript in preparation. [60]

In this collaborative work, me and a colleague from Linköping University propose a two-dimensional model for types and levels of automation in Visual Analytics. The work grounds on the existing body of literature on Levels of Automation, as well as on the theoretical framework for the Visual Analytics pipeline. In this work, I contributed with my knowledge of Levels of Automation taxonomies. I also initiated 
the idea of creating the model, which was later elaborated collaboratively. I took an active role in writing the article.

Veronika Domova and Shiva Sander-Tavallaey. "Visualization for quality health-care: patient flow exploration". In: IEEE International Conference on Big Data. IEEE. 2019 [57]

In this collaborative work, me and a colleague from ABB CRC together with two municipal Swedish hospitals and several industrial partners investigated possibilities of optimizing patient flows and resource allocation in Swedish hospitals. In this work, I was an active participant in all the project stages including field studies, brainstorming, design and development, and evaluation. I was working on the design and development of the web portal. I took the leading role in writing the article.

Veronika Domova, Alvaro Aranda Munoz, Elsa Vaara, and Petra Edoff. "Feel the Water: Expressing Physicality of District Heating Processes in Functional Overview Displays". In: ACM International Conference on Interactive Surfaces and Spaces. ACM. 2019 [55]

In this collaborative work, me and several colleagues from RISE explored alternative data presentation means beyond conventional industrial process graphics. One particular aspect of the research was related to visually conveying the physicality of the underlying industrial processes. The work resulted in three interactive prototypes of novel visualizations. In this work, I have designed and developed two out of three prototypes. I have participated in all the stages of the design process, i.e. field studies, analysis, brainstorming, concept development, and evaluation. I took the leading role in writing the article.

Veronika Domova and Goranka Zoric. "Towards Effective Industrial Robot Fleet Visualization for Remote Service Applications". In: Enabling Technologies: Infrastructure for Collaborative Enterprises (WETICE), 2017 IEEE 26th International Conference on. IEEE. 2017, pp. 185-190 [61] 
This work presents a novel web-based interface for remote monitoring of a large fleet of industrial robots. The interface combines several interactive data layouts to maximize effective data presentation. I was the main contributor to the concept design and the implementation of the interface. I was actively involved into communication with the project's stakeholders to agree on the preferable designs and future directions. I also took the leading role in writing the article.

Veronika Domova, Maria Ralph, Elina Vartiainen, Alvaro Aranda Muñoz, Adam Henriksson, and Susanne Timsjö. “Re-Introducing Physical User Interfaces into Industrial Control Rooms". In: Proceedings of the European Conference on Cognitive Ergonomics 2017. ACM. 2017, pp. 162-168 [56]

This work presents two tangible devices, the tactile mouse and the shift report button, aiming to a) re-introduce the physicality into industrial control rooms and b) facilitate the user's interaction with the control station. I was the main contributor to the tactile mouse concept, starting from the idea to the high-fidelity implementation of the device and the required software. The idea of the shift report button was created in collaboration with several other participants of the project. I took over the first iteration of the prototype and elaborated it to be a fully functional solution. The final look-and-feel, i.e. the 3D-printed version, of the devices was developed by another participant in the project. Also, I developed the user interface needed for evaluation of both devices. I took part in the evaluation tests of both prototypes in the field. I also took the leading role in writing the article.

Veronika Domova and Aldo Dagnino. "Towards intelligent alarm management in the Age of IIoT". in: 2017 Global Internet of Things Summit (GIoTS). IEEE. 2017, pp. 1-5 [53]

In this work on Visual Analytics, I proposes an interface for the analysis of sequences of industrial alarms. Using the proposed concept, the analyst can differentiate informative alarms from redundant notifications with the purpose of reducing the number of alarms delivered 
to industrial operators. My major contribution is the design and development of the visualization concepts and development of the highfidelity prototype of the visualization system. I also took the leading role in writing the article.

Goranka Zoric, Veronika Domova, Maria Ralph, Elina Vartiainen, Petra Björndal, and Alvaro Aranda Muñoz. "Supporting maritime remote experts working over distance". In: Proceedings of the 9th Nordic Conference on Human-Computer Interaction. ACM. 2016, p. 124 [250]

This work proposes an interactive web-portal to support maritime remote experts in their daily routines. I was an active member in all the project stages, i.e. field studies, brainstormings, user evaluations, etc. My major contribution is the design and development of the highfidelity prototype of the web-portal. I also took an active role in writing the paper.

Veronika Domova, Saad Azhar, Maria Ralph, and Jonas Brönmark. “Untethered Workspaces: A Zones Concept Towards Supporting Operator Movements in Control Rooms". In: Proceedings of the 2016 CHI Conference Extended Abstracts on Human Factors in Computing Systems. ACM. 2016, pp. 680-689 [52]

This work introduces the concept of interactive zones into an industrial control room. Depending on the zone, the operator can interact with the control station by different interaction means. In collaboration with other participants of the project, I contributed to the overall zones concept. I was the main developer of the high-fidelity prototype of the final solution. I also took the leading role in writing the paper. 


\subsection{The complete list of my academic publications}

[50] Veronika Domova. "Fidgeting with the Environment: a Tangible Control for Interacting with a Smart Light". Submitted.

[51] Veronika Domova. “Guiding the Operator's Attention Among a Plurality of Operator Workstation Screens". Submitted.

[52] Veronika Domova, Saad Azhar, Maria Ralph, and Jonas Brönmark. "Untethered Workspaces: A Zones Concept Towards Supporting Operator Movements in Control Rooms". In: Proceedings of the 2016 CHI Conference Extended Abstracts on Human Factors in Computing Systems. ACM. 2016, pp. 680689.

[53] Veronika Domova and Aldo Dagnino. "Towards intelligent alarm management in the Age of IIoT". In: 2017 Global Internet of Things Summit (GIoTS). IEEE. 2017, pp. 1-5.

[54] Veronika Domova, Erik Gärtner, Johan Källström, Martin Pallin, Fredrik Präntare, and Nikita Korzhitskii. "Improving Usability of Decision Support Systems for SAR Operations: WARA-PS case study". Submitted.

[55] Veronika Domova, Alvaro Aranda Munoz, Elsa Vaara, and Petra Edoff. "Feel the Water: Expressing Physicality of District Heating Processes in Functional Overview Displays". In: ACM International Conference on Interactive Surfaces and Spaces. ACM. 2019.

[56] Veronika Domova, Maria Ralph, Elina Vartiainen, Alvaro Aranda Muñoz, Adam Henriksson, and Susanne Timsjö. "Re-Introducing Physical User Interfaces into Industrial Control Rooms". In: Proceedings of the European Conference on Cognitive Ergonomics 2017. ACM. 2017, pp. 162-168. 


\subsection{The complete list of my academic publications}

[57] Veronika Domova and Shiva Sander-Tavallaey. "Visualization for quality health-care: patient flow exploration". In: IEEE International Conference on Big Data. IEEE. 2019.

[58] Veronika Domova, Elina Vartiainen, Saad Azhar, and Maria Ralph. "An interactive surface solution to support collaborative work onboard ships". In: Proceedings of the 2013 ACM international conference on Interactive tabletops and surfaces. ACM. 2013, pp. 265-272.

[59] Veronika Domova, Elina Vartiainen, and Marcus Englund. "Designing a remote video collaboration system for industrial settings". In: Proceedings of the Ninth ACM International Conference on Interactive Tabletops and Surfaces. ACM. 2014, pp. 229-238.

[60] Veronika Domova and Katerina Vrotsou. “A Model for Types and Levels of Automation in Visual Analytics: Examples from Event-Sequence Analytics". Manuscript in preparation.

[61] Veronika Domova and Goranka Zoric. "Towards Effective Industrial Robot Fleet Visualization for Remote Service Applications". In: Enabling Technologies: Infrastructure for Collaborative Enterprises (WETICE), 2017 IEEE 26th International Conference on. IEEE. 2017, pp. 185-190.

[140] Lawrence H. Kim, Daniel S. Drew, Veronika Domova, and Sean Follmer. "User-Defined Swarm Robot Control". In: Proceedings of the 2020 CHI Conference on Human Factors in Computing Systems. CHI '20. Honolulu, HI, USA: Association for Computing Machinery, 2020, pp. 1-13.

[228] Elina Vartiainen, Veronika Domova, and Marcus Englund. "Expert on wheels: an approach to remote collaboration". In: Proceedings of the 3rd International Conference on Human-Agent Interaction. ACM. 2015, pp. 49-54. 
[250] Goranka Zoric, Veronika Domova, Maria Ralph, Elina Vartiainen, Petra Björndal, and Alvaro Aranda Muñoz. "Supporting maritime remote experts working over distance". In: Proceedings of the 9th Nordic Conference on Human-Computer Interaction. ACM. 2016, p. 124. 


\section{Chapter 2}

\section{Theoretical framework}

In the scope of my $\mathrm{PhD}$ research, I have conducted a broad literature study in a variety of different domains. Directly or indirectly, these domains are related to designing interactive systems for industrial process control. In this chapter, I will give a short summary of the related literature discovered. I start the overview with different taxonomies of industrial automation. Different levels of automation lead to different interaction setups between the human and the system and have their influence on the design of the system. Next, I provide some background theory related to situation awareness, an integral aspect of human-automation interaction which is tightly correlated with the system's level of automation. I proceed with an overview of the specifics of human perception and performance. Deliberately I focus on humans' weaknesses and biases to highlight their imperfection when dealing with large amounts of data, making decisions in stressful conditions, and interacting with automation. My goal is to emphasize the need for supporting tools and technologies that would diminish these weaknesses and capitalize on humans' strengths. I then review approaches to data presentation and design of interactive spaces. First, I focus on theoretical frameworks and best practices in both domains, then I list relevant solutions developed for industrial control rooms. 


\section{THEORETICAL FRAMEWORK}

\subsection{Levels of Automation}

Automation is application of mechanical, electronic, and computerbased technologies to operate and control manufacturing with the aim to reduce the amount of involved manual effort in product design, production planning and control, and business functions of the company [93]. Parasuraman et al. [178] refer to automation as the full or partial replacement of a function previously carried out manually by a human operator.

Arguably, the most widely applied taxonomy, which underpins many attempts to build intelligent industrial systems, is known as levels of automation (LOA). Essentially, levels of automation mean allocation of tasks between humans and automation ranging from entirely manual operations to fully automated. There is a rich history of research on the topic of LOA. Early efforts in this direction started with Fitts' intent to provide a basis for appropriate allocation of system functions to a human or machine for reaching effective system performance [77], i.e. an attempt to create a "Men-Are-Better-At/Machines-Are-BetterAt" (MABA-MABA) list. This and other similar MABA-MABA lists [37. 65] generally emphasize that people and computers have their strengths and weaknesses, and task allocation in systems should be designed in a way to capitalize on the strengths and compensate for or eliminate the weaknesses. Many have taken such lists as a proper scientific basis for distributing functions between humans and machines [200]. However, MABA-MABA lists were also widely criticized a) for the extent to which they tend to frame human capabilities in machine terms [200], b) for being static whereas real-world tasks are dynamic [98], and c) in general, for comparing men and machines instead of seeing them as complimentary [126]. Moreover, soon it became clear that introducing automation does not eliminate the human weaknesses, but rather leads to new human strengths and weaknesses, often unanticipated [11]. 
With respect to models of man-machine interaction, one of the most cited works, perhaps, is the technical report by Sheridan and Verplank [202] that described a variety of ways in which humans and computers can interact in undersea operations, i.e. ten levels of automation. Their taxonomy has been extensively discussed throughout the literature and served as the foundation for several subsequent taxonomies of LOAs including reformulations of levels focusing on particular domains or aiming at broader types of automation [70, 69, 127, 136], several surveys provide a comprehensive overview of existing LOA taxonomies [226, 82].

Sheridan [200] criticized the taxonomy for oversimplicity advocating that on different stages of any rather complex task the suitable level of automation is likely to be different. Endsley [69] proposed that there are four generic functions in a human-machine system that either the human or the automated system performs, i.e. monitoring, generating, selecting, and implementing. She then formulated a tenlevel taxonomy by assigning these functions to the human or computer or a combination of the two. Parasuraman, Sheridan, Wickens et al. [178, 43] suggested a two-dimensional taxonomy where the second dimension is the four stages of human information processing, i.e. information filtering, information integration, decision, and action implementation, derived from the classic human information processing models [28, 29, 78]. Regarding the levels of automation within each stage, Wickens [237] argues that the particular number of levels is in a way subjective, but what actually matters is the relative order of levels, i.e. the movement up or down in automation.

Being based on function allocation, the main question LOA approaches are trying to answer is what should be done by the human and what should be automated [178]. Earlier, there was a tendency to perceive human operators as a major source of variation and unpredictability in system performance, therefore, many designers believed that humans should be eliminated from the system [15]. Consequently, the following principle of automation was prevalent: auto- 
mate as many functions as technology permits, let the human pick up whichever functions are left over. In 1980s, the idea of "lights-out factories", i.e. unmanned factories with fully automated production [32], was highly popular and propagated. New technology was introduced simply as a substitution of machines for people allowing to preserve the original system setup while improving its KPIs, e.g. achieving lower workloads, better economy, fewer human errors, higher accuracy [107]. Such an approach led to automating standard processes within clearly defined and understood conditions [75]. The resulting automation, however, was prone to failing in an unexpected manner in off-nominal conditions, putting operators in a trouble due to inappropriate feedback and poor interaction means [172].

Later on, multiple authors have expressed a critique of LOA-based approaches and high levels of automation. First of all, it became clear that introducing automation does not eliminate human weaknesses, but rather leads to new human strengths and weaknesses, often unanticipated [11]. Furthermore, the ultimate goal of LOA-based methods is full automation [205] which dictates interaction means [122] and suggests that increases in automation come at the cost of reducing human control, i.e. designers must decide between providing control to the human or involving computer automation [205]. Finally, an increased level of automation causes the human-out-of-the-loop problem meaning the inability of the human to take over control in the case of a failure [73].

In response to the weaknesses of LOA-based approaches, several alternatives have been proposed. In the early 90 s, authors became more open to the idea of human-centered automation [23], which promoted keeping the human as the main authority while automation technology continued its penetration to all spheres of life. Sheridan [201] listed 10 alternative setups on how human-centered automation could be arranged in practice. However, he considered them rather idealistic and advocated that human-centered automation remains as "a kind of romantic ideal" which is not fully achievable at this point [200]. End- 
sley and Kiris [73] advocated the usage of a lower LOA, which could maintain the human operator in the loop and ensure they are able to perform tasks manually when needed. Another popular idea is the socalled adaptive automation which assumes dynamic function allocation that can change in real-time based on the context [162, 196]. While most authors assumed that the automated solution should decide on its own how to adapt its own behavior and which level of automation to choose, some authors advocated adaptable technology where the human decides how much automation to use [196]. Shneiderman [205] argues that it might be reasonable to fully automate some tasks, while in the case of others there might be value in granting full control to the human. However, the new goal is to seek possibilities to combine full human mastery and high levels of automation, i.e. create highly automated systems that would also augment and empower people. For this purpose, Shneiderman proposes a two-dimensional model of automation and control that disconnects the level of human control from the extent of computer automation, emphasizing that high levels of human control and high levels of automation can co-exist by means of well-thought design. All in all, during the last decades, more and more authors encourage focusing on how to support the teamwork between the human and the automation [79, 143 , 123, 122]. The main difference of these approaches compared to LOA is that the collaborative nature defines the necessary automation and shapes the human-automation interaction [122].

\subsection{Situation awareness}

Situation awareness (SA) has been widely recognized as a crucial basis for effective decision-making in modern industrial systems [72, 191]. Authors agree that a major portion of today's operator job becomes that of obtaining and maintaining good SA [8, 34, 241]. SA generally refers to the knowledge about the status of the system. In the literature, several models of SA have been developed [3, 63, 216] shar- 


\section{THEORETICAL FRAMEWORK}

ing many similarities in terms of their focus on the key-terms, such as mental models and attention. Arguably, one of the most extensive and cited models of SA is the one introduced by Endsley, who defined SA as "the perception of the elements in the environment within a volume of time and space, the comprehension of their meaning, and the projection of their status in the near future" [72]. The model comprises three ascending levels of SA, namely:

- Level 1: perceiving the status of the system, i.e. its attributes and dynamics of its composites.

- Level 2: comprehending the meaning of the information gained in the previous step, i.e. forming an understanding of the holistic picture of the system.

- Level 3: predicting the future status of the system.

Endsley [72] emphasizes that SA is a state of knowledge which is separate from situation assessment, decision making, and performance. She motivates this statement by the fact that even the best-trained decision makers will make wrong decisions once they have insufficient SA; similarly, a person with perfect SA might still make mistakes, e.g. due to insufficient training.

An accurate mental model is one of the prerequisites for achieving sufficient SA [68]. A mental model is a collection of "well-defined, highly organized yet dynamic knowledge structures" about the system in focus that develop over time based on the user's experience [89]. People generally tend to think in mental models when working with systems or devices [236]. Mental models form based on humans' prior experience, assumptions, and the direct experience with the item in focus; mental models can change. People refer to mental models to estimate the system's state and predict its next action, or figure what they should do with it. 
Too much data can overwhelm the user to attend, process, comprehend, and integrate the presented information in an efficient manner, resulting in information overload and negative effects on their SA [71]. There is a common agreement that an industrial system interface should support and enhance the user's sufficient SA [191, 99]. Endsley [72] formulated a set of requirements to interface design for enhancing SA. They incorporate the demand for efficient data layouts and presentation that would a) facilitate the 2nd and 3rd levels of SA, b) allow information organization in correspondence with the operator's personal goals, c) allow aggregating information from multiple sources on one view to enable parallel rather than sequential information processing. The system should also reduce the need to do manual calculations, employ indicators and visual cues able to capture the human's attention, and provide projections of future events and states to support the 3rd level of SA. Training is another widely accepted approach to improving situation awareness of industrial personnel [192]. For example, Gaba et al. [84] advocate that many aspects of SA can be improved by practicing, e.g. scanning displays to maximize perception, training pattern recognition, practicing multitasking, exercising attention allocation, etc.

The notion of LOA is tightly connected to the notion of SA, see Figure 2.1. Some empirical research shows that, as the level of automation grows, the performance of the human-automation system linearly improves and the human's workload proportionally reduces [176], however, the human's ability to response to unexpected system failures, as well as SA, decline. The decline in situation awareness is often referenced as human-out-of-the-loop problem [73]. This is related to the fact that the more automated and reliable the system is, the less visual attention it will receive and hence degrade the operator's situation awareness at Endsley's Level 1, i.e. noticing [238]. Another potential reason for worsening of SA is the so-called generation effect [213] when humans tend to allocate greater mental resources for actions and their consequences that they undertake themselves com- 


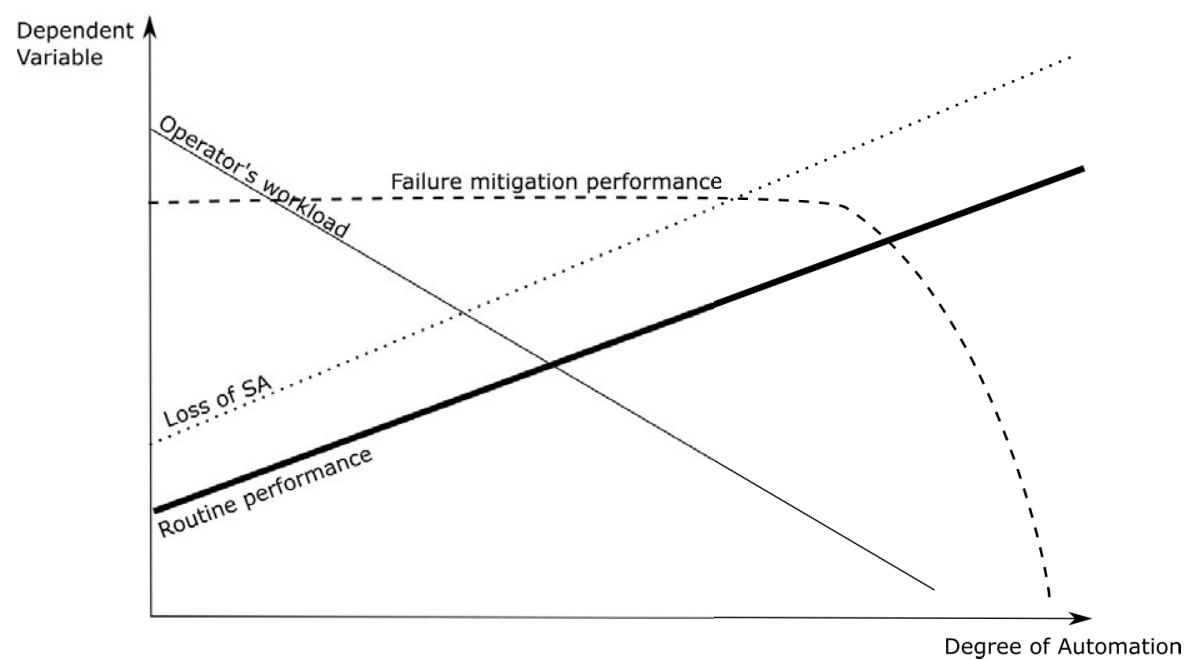

Figure 2.1: Functions of the four key variables as the level of automation increases. The image is adapted from [237].

pared to when they are witnessing the actions being performed by another agent, whether a human or automation. With respect to industrial control, this phenomenon degrades Level 2 situation awareness and compromises the ability to take over the control in a timely and efficient manner should automation fall or fail to act according to the human's expectations [199].

\subsection{Human perception and attention}

Existing literature on human mental processing reports that, every second, humans receive about 40 billion sensory inputs, but consciously only aware of around 40 of them [236]. About $80 \%$ of the stimuli arrives through the sight, hearing delivers about $10 \%$, the remaining $10 \%$ of information is received through smell, haptic, and taste senses sensors [159]. The human visual system has a binocular field of view exceeding 180 degrees horizontally and 150 degrees 
vertically [88]. The visual field comprises focal and peripheral areas; the first one is accentuated and is the most critical for specific object recognition, the second one is attenuated and is used for getting the gist of a scene [145, 150]. The focal area allows the greatest visual acuity as it senses detail, color, and textures. Peripheral vision is attuned to sensing contrast and motion, it tends to guide the focal vision to informative stimuli [4]. The human haptic system offers an independent sensory channel which is processed by the brain to enhance the human's experience in a multimodal environment [95]. Hairless skin, such as that on palms and fingertips, is highly sensitive to detailed tactile information with the extent of sensitivity depending on a variety of the body physiology factors and stimulation types; hairy skin is not capable to effectively perceive detailed texture or the specific geometric structure of a surface or object, but is efficient in detecting vibrations and static forces [95]. A body of literature [121, 168, 33] investigated haptic tactile skin mechanoreceptor characteristics and revealed particular numerical resolutions of their sensitivity.

Humans tend to selectively attend to some stimuli in preference to others, furthermore, there is always an aspect of the amount and intensity of attention paid to one or another stimulus [129]. According to Berlyne [17], the intensity of attention is proportional to the level of arousal which is largely defined by the properties of the stimuli; certain properties, such as novelty, complexity, and incongruity, are more arousing than others. Such arousing object properties are especially relevant to an involuntary selective process; in voluntary attention, the human attends to stimuli because of their relevance to a task they are performing rather than due to their arousing qualities [129].

Attention is essentially a limited resource, it can only operate on one stimulus or one response at a time. When two stimuli are presented at once, often, only one of them is perceived, while the other is dropped or attended after the analysis of the first one has been completed [30]; if both are perceived, the responses that they trigger are often made consequently rather than simultaneously [48]. Furthermore, it is rel- 
atively easy to focus attention exclusively on a single object and difficult to spread attention among several target objects. On the other hand, it is natural to detect several aspects of an object, but it is rather difficult or even impossible to prevent noticing irrelevant attributes, e.g. perceive the color of an object without seeing its shape [129]. Moreover, sustained attention can last about ten minutes at most, after that time it starts to wane [236].

In addition to attention constraints, there are multiple cognitive effects due to which humans may become effectively blind to notifications from the environment. First of all, this situation might occur due to the mental state called flow when the user is fully engaged in some ongoing activity, often demanding a high degree of skill and commitment, ignoring the surroundings [129, 45]. Another cognitive effect is the so-called tunnel vision which occurs when peripheral stimuli are not responded to because they are not seen. It can be the effect of stress or it can be caused by the fundamental nature of the task, see cognitive tunnelling [161], or because of too high demands for mental resources of higher level mental tasks [185]. Mind wandering is another common phenomenon that influences concentration of attention. It refers to occasionally fading into thinking about something secondary when performing a task. The literature suggests that, during everyday activities, the human's mind wanders up to $30 \%$ of the time, and in some cases, such as driving on an uncrowded highway, it might reach up to $70 \%$ [236]. When mind wandering occurs, attention shifts lead to failures in the main task performance and superficial representations of the external environment [215]. Another common effect is the socalled change blindness which refers to the inability of a person to detect changes in the environment after an interruption or a deviation in attention occurred [189]. To name more, expectations of frequency affect attention, i.e. when people expect something to happen with a certain frequency, they would often miss the occurrence if it happens more or less often compared to their expectations [16]. 
With respect to industrial interfaces, the literature has widely identified the problems with attention as key reasons for poor SA in automated systems [125]. It was proposed to mitigate change blindness by guiding the user's attention to changes [36] and explaining them [14]. Interactive visual representations of historical events were found effective in mitigating change blindness after interruptions, for example, when interactive event logs are presented in tabular format [214] and as interactive graphical event timelines [193]. Deatherage [47] advocates that, for improved detection, some signals are better suited for auditory than for visual presentation and vice versa, he provides implications for design and particular recommendations for sound notification properties. Heun et al. [104] experimented with adaptation of interfaces to the peripheral vision of the user with the goal to help the user to monitor and operate real-time large data sets; in their interface, highly detailed information is provided to the user in their focal field of vision, the information in the periphery is abstracted and is only highlighted in case of important data changes. Renner and Pfeiffer [188] worked on AR-based assistance systems intended to guide the visual attention of the user towards the next relevant item in manual assembly scenarios. Their experiments showed that the conventional arrow-based guidance technique was the most efficient and got the best ratings from the study participants.

\subsection{Human performance}

The human capacity to perform mental work is generally limited. Numerical estimates of the capacity of visual attention and working memory show that only a limited amount of items can be noticed, processed and kept in memory at the same time [42, 157, 198]. For example, various experiments show that people can remember and process only four pieces of information at a time [7, 44]. Memorized facts tend to degrade rather quickly in humans' memory following the 
so-called Forgetting Curve [165], which is a rather natural process to free up mental resources for new information.

Different actions impose different demands, i.e. loads, on the limited capacity of mental resources. The theory says that there are three kinds of loads that can be made on a person: cognitive, visual, and motor, listed in the order from the most to the least "expensive" [236]. When there is not enough capacity of mental resources to meet the demands of an activity, it will likely falter or fail [129]. On the other hand, well-practiced skills might not require much mental resources and might be performed automatically even allowing nearly multitasking [236]. Essentially, people cannot multitask, but they can switch fast between tasks [236]. One possible exception exists, namely when someone is proficient at doing a physical task, then they can do it simultaneously with a mental task, e.g. walk and talk or read at the same time. However, there is evidence of worsened performance in the case of such multitasking, for example Hyman et al. report that people talking on the phone while walking collided with other people more often and did not notice what was around them [112].

According to the Yerkes-Dodson law [243], a little stress, i.e arousal, can help perform a task, especially if it is simple; too much stress, on the contrary, degrades performance especially in complex tasks and leads to mistakes. People generally tend to make mistakes due to various reasons, e.g. lack of knowledge or lack of attention, poor situation awareness, wrong mental model, etc. The literature suggests that "humans are generally prone to fallible heuristics and various decision biases that are heavily influenced by experience, framing of cues, and presentation of information" [46]. For instance, confirmation bias occurs when people explicitly look for information to confirm their prior assumption and disregard facts that contradict this assumption. Similarly, assimilation bias occurs when a person tries to assimilate newly received information to fit into a pre-existing mental model even though the information obviously contradicts the model. Morrell [67] classifies errors into two types, i.e. performance errors, i.e. 
errors in the task procedure, and motor-control errors, i.e. incorrect physical actions. He divides performance errors further into commission errors, i.e. unnecessary extra actions, omission errors, i.e. missing actions, and wrong action errors, i.e. a timely action but of a wrong choice. Human errors tend to have an accumulative effect, each error makes a hole in the system leading to lots of holes, eventually, yet another human error results in a mishap [186]; the described situation is known as the so-called Swiss cheese model. People also tend to use different strategies when dealing with errors, namely a) systematic exploration, i.e. proper planning what actions should be used for correcting an error, b) trial and error, i.e. random actions, c) rigid explorations, i.e. performing the same action over and over again regardless of its inability to solve the error [130].

The presence of automated decision aids can now and then push the user "into the general human tendency to travel the road of least cognitive effort" [212, 128]. Individual humans, as well as groups, demonstrate a tendency toward the so-called automation bias, i.e. "the use of automation as a heuristic replacement for vigilant information seeking and processing" [212], which happens when the human operator or does not make an effort to find contradictory information to question a computer-generated solution but instead accepts it as correct. Errors caused by automation bias have been reported in many different sources, some of the examples are described in [46]. Humans tend to follow automated recommendations even when they contradict their training or other $100 \%$ correct and presented indicators; humans make errors of omission and commission on more than $50 \%$ of the cases providing such an opportunity [212, 211]. Skitka et al. [211] concluded that a) when the automated aid system is prone to mistakes, people tend to make more omission errors compared to when automated aids are not available; this is valid even when people are aware that the system is not trustworthy; and b) on the contrary, when the automated aid system works properly, people make fewer errors when using it than when not having it. The literature suggests that 


\section{THEORETICAL FRAMEWORK}

humans demonstrate better performance when a) autogenerated recommendations come together with a reliability rating, and b) when people are trained with a focus on automation bias and associated errors; the latter however reduces commission, but not omission errors [212].

\subsection{Information visualization}

Bertin [19] placed visualization among the main "languages" related to information processing as "graphic representation constitutes one of the basic sign-systems conceived by the human mind for the purpose of storing, understanding, and communicating essential information". Information visualization is the process of representing data visually in a meaningful way that facilitates the user's understanding [235]. Information Visualization has a variety of methods for the visualization of data starting from conventional visualization techniques such as $\mathrm{x}$-y plots, maps, line-charts, histograms, and up to new visualization approaches such as Parallel Coordinates [113], Treemaps [207], and Pixel-based [135] data representations.

With respect to dealing with large amounts of data, one popular browsing and searching strategy is Shneiderman's Visual Information Seeking Mantra [206], which promotes to show a high-level overview of the data first, then support zoom and filtering activities, and finally provide access to required details on demand. In addition, large volumes of multidimensional data often require certain pre-processing before the actual visualization, e.g. clustering or dimension reduction [41].

Starren and Johnson [217] categorized data visualization approaches into five large groups, i.e. generated text, lists, tables, graphs, and icons. Bui and Hsu [31] elaborated the taxonomy arranging it according to increasing levels of graphical abstraction and illustrated it with real-world examples. According to the taxonomy, the lowest level of 
sophistication incorporates lists and tables that normally show textual and numerical information in a rather straightforward manner. The authors emphasize that, although precise, such information presentation fails to facilitate comprehension of subtle correlations and trends, especially in the case of large and complex datasets. The next abstraction level involves different types of plots and charts, i.e. the visualization means emphasizing relative values, facilitating comparisons and understanding of data trends. Graphs and trees are examples of a further degree of abstraction as they expound conceptual relations between visual components. Finally, pictograms, i.e. icons, maps, diagrams, and images, are considered to be the top echelon of this hierarchy as they target to be graphical representations of complex realworld concepts, entities, and phenomena observed in the domain.

With respect to industrial data visualization, a body of literature discusses several approaches, namely, schematic interfaces, dashboards, and functional visualizations. Multiple works, see for example [97. 183] evaluate existing schematic interfaces, and outline multiple usability issues such as cluttered views, presence of non-relevant information, inconsistency of design, etc. Reising and Bullemer [187] outline two drawbacks of schematic layouts, namely 1) the loss of valuable display space to static information, e.g. equipment icons, and 2) absence of contextual information about the process variables, such as thresholds, optimal values, or deviations, that would support the operator's understanding of the visualized value.

During the last decades, dashboards became an extremely popular means for consolidating and arranging the most important information from different sources on a single screen, so that it can be monitored at a glance [76]. Information in the dashboards can be arranged into different layouts. A common approach is to group visual indicators into dedicated thematic areas which are often visually outlined. The information on a dashboard can be depicted by different visual means, e.g. graphical objects, numeric indicators, maps, tables, timelines, charts, etc. A tabular layout of numerical indicators is probably 
the most pragmatic and the fastest approach to create an overview dashboard. However, such solutions often become very cluttered and visually monotonous making it hard for the operator to read and comprehend the presented information at-a-glance [187].

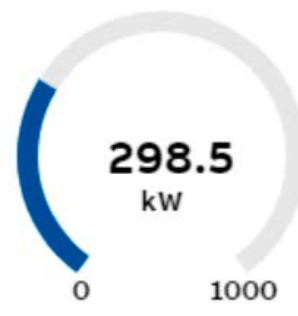

Figure 2.2: A variation of a gauge. Perhaps, gauges are the most widely used examples of functional visualizations.

Functional visualizations, see Figure 2.2, employ qualitative graphical indicators promoting direct perception. Perceptual organization principles, see for example [182, 177], are used to present the information in a form that is easy for humans to pick up. In the literature, there is growing empirical evidence indicating that operators' SA improves significantly when they monitor the production process presented using functional visualizations compared to schematic data presentation. For example, in [223] Tharanathan et al. evaluated operator performance using two overview displays presenting the same information in two different formats, namely, in a functional design and a schematic design. Their results showed that the operators' SA improved significantly when they followed the production process using the functional display compared to the schematic display. In [170], Noah et al. present and evaluate three alternate functional visualizations, i.e. surface chart, heat map, and visual thesaurus, with respect to their effectiveness in supporting an industrial operator's performance and SA. The obtained results show that the surface chart display, i.e. a monolith functional visualization, was superior to the other two options. A potential reason for the detected higher performance 
could be that the visualization uses a single integrated graphical object which, according to the literature, facilitates the user's understanding.

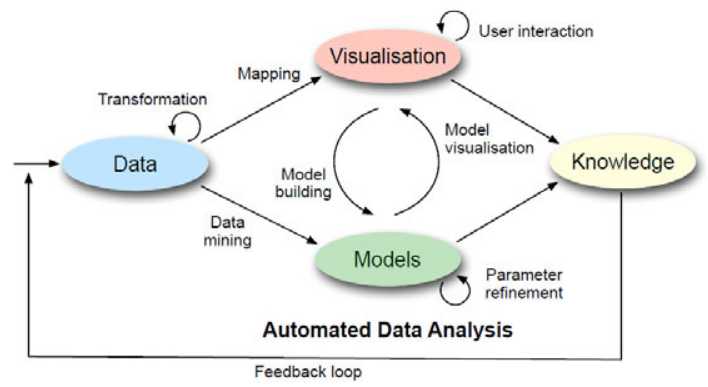

Figure 2.3: Visual Analytics pipeline proposed by Keim et al. [134]

Visual Analytics (VA) is "the science of analytical reasoning facilitated by interactive visual interfaces" [224]; it combines automated data mining techniques with information visualization to facilitate understanding, reasoning and decision making when dealing with large and complex data sets [133]. VA combines human and artificial intelligence enabling the collaboration between the natural human abilities of sense-making and reasoning and the computational power of data mining tools [20]. VA combines techniques from the fields of data processing, information visualization, human-computer interaction, and human reasoning [149]. Perhaps the most cited VA pipeline is the one presented by Keim et. [134], see Figure 2.3. It presents the VA process as an abstract outline with four major stages:

- Data: incorporates data pre-processing, e.g. transforming it to a suitable form, removing redundancy, errors and invalid items, merging several sources of data, etc.

- Visualization: incorporates visual exploration of the data presented e.g. in an interactive visual interface.

- Models: incorporates automatic analysis that results in generated data models and detected patterns. 


\section{THEORETICAL FRAMEWORK}

- Knowledge: incorporates reasoning, i.e. knowledge generation based on the patterns and models discovered in the previous step.

With respect to industrial data analytics, many authors agree that one of the main challenges is efficient visualization [240, 163, 39]. Wang et al. in [233] acknowledge that a value of an efficient industrial data visualization could inspire new ideas for solving problems, help in decision making, and indicate the correlation or causality between different events.

\subsection{Interactive spaces}

Drawing on the principles of Phenomenology, Dourish [62] argues that, with relation to both physical and social aspects, our experiences cannot be separated from the reality of our bodily presence in the world because our nature as social beings assumes constant acting and interacting. Against this background, it is only natural to assume that the environment where one is performing their work plays a crucial role and influences heavily their actions and workflows. Similarly, interaction with computer systems is intimately connected with the settings in which it occurs. Conventional human-computer interaction means, i.e. based on keyboards, mice, screens, windows, menus, icons, and pointing, are often said to not reflect the connection with the environment as they are based on metaphors originating from technology [227, 62]. Alternatively, the so-called embodied interaction, according to Dourish [62], allows "possessing and acting through a physical manifestation in the world". Designing embodied interaction assumes incorporating the knowledge about the environment in the interaction design which requires understanding how such knowledge can be shaped through physical manifestations to support construction of meaning within the interaction [100]. 
According to phenomenologists, the world is filled with meaning, which it reveals to us by means of being available for our actions [62]. With respect to human-computer interaction, the designer should explicitly communicate to the user how the design is intended to be used [62]. In relation to this, Gibson [87] introduced the concept of affordance describing it as action possibilities in the environment. Norman [171] elaborated this idea further into the notion of perceived affordances meaning that if one wants people to take action on an object, either real or digital, it is essential that the users can easily understand the object and figure out what they can and should do with it. When designing the environment for a task, one should make sure that the objects in the environment are easy to see, easy to find, and that they have clear affordances [236].

With respect to embodied interaction, several research directions can be outlined in the literature. A body of work, e.g. [148, 12], exists on the topic of proxemic interaction, which explores the interaction between users and computers grounding on Hall's foundational research on proxemics [96]. The latter describes people's proxemic behavior with regard to their interpersonal communication, i.e. how they use space to mediate their intentions and communicate with other people. Natural interaction is another popular topic of research in this direction. The concept of natural interaction grounds a) on people's natural ability to communicate with others through gestures, speech, gaze, and body movements, and b) on people's tendency to explore the surroundings by manipulating physical objects. The main assumption of natural interaction is that people should be enabled to interact with technology in the same way how they interact with the surroundings in their everyday life, i.e. in a spontaneous and free manner [227].

Haptic interaction is another integral part of interactive spaces which draws on the concepts of embodiment. It covers sensation- and perception-related aspects of touch, as well as motor and cognitive aspects of body movement [95]. With respect to human-computer in- 
teraction, haptic interaction is believed to improve reaction time and decrease the probability of hand-eye coordination issues in computerrelated tasks [95]. The existing literature in psychophysical research [121, 168, 203, 33, 22], elicited a set of design guidelines for tactile interaction including numerical values of intensity of stimuli, their periodicity, and optimal stimulation locations for various stimuli.

Tangible interaction is another popular topic of research exploring interactions between humans and physical objects augmented with some kind of computation. Research in this field typically leads to novel tangible artifacts with an emphasis on physical form and tactile interaction [13], e.g. sliders with haptic feedback [154], pressuresensing flexible tubes [222]. Dourish [62] argues that tangible computing resides on the principles of embodiment; it allows the user to a) better communicate the meaning of their action and b) incorporate the technology into their world and everyday practice as compared to the conventional keyboard- monitor- and mouse-based interactions that are rather rigid and restrictive.

In the general interaction design literature, there is a body of work related to haptic and tangible interaction. With respect to office environments, a body of research tried to augment standard keyboard-mousescreen interaction and introduce novel interaction means. Some mouse prototypes introduce novel input modalities, e.g. provide more freedom of movements [158, 180], others provide visual [242] or haptic feedback [179, 147]. Various extensions to keyboards were proposed to a) recognize gestures [221, 245], b) sense pressure inputs [49], c) provide visual [24] or d) haptic feedback [9, 195]. Shape-changing versions of mice [141], displays [167], buttons [225, 138, 219], and keyboards [9] were proposed. Many authors investigated potential benefits of actuated desktop interfaces; in such interfaces, some composites can move in a manner that can be noticed by the user and benefit them in some way, e.g. actuated mice and keyboards [10, 142], actuated monitors [204], actuated desktops [152], actuated desktop devices, such as swarm robots [139, 140] and interactive lamps [175]. 
Within the industrial domain, there is a limited body of work focusing on alternative interaction means in industrial control rooms. Choi et al. in [40] investigated opportunities of using speech and gestures to support traffic control operators. Heimonen et al. [102] present a multimodal system that enables the operators to interact freely through gestures and voice; they emphasize that alternative interaction means should a) provide the feeling of control and safety and b) enable the operators to access information from any location in the control room. Fuhrmann and Kaiser [83] present an interaction concept for future control rooms that comprises a large overview display, a portable mobile interface, a gesture recognition system, and an audio system for emitting directed sounds. Sirkka et. al [209, 208] and Frimalm et al. [81] conducted research on providing audio cues that could help the operators to differentiate between the type and severity of alarms and system status changes. In [74], Fagerlönn et al. developed a multimodal alarm display combining visual representations with spatially presented auditory icons.

With respect to tangible interaction and tactile data presentation in the industrial domain, only a limited body of work is available. Ressler et al. [190] developed a virtual environment and a set of active haptic devices that can be used to control it, as well as for getting feedback; the actions in the virtual environment are mimicked in the real world and vice versa. In the design experiment by Jensen et al. [119], a group of design students remodeled the interface of an industrial motor controller to incorporate new movement-based interactions with the focus on physicality. In [210], Sitorus et al. developed a set of tangible prototypes to assist technicians in configuring complex industrial systems; in the design process, they focused on physical aspects of the systems and the skills and experiences of the technicians. Müller et al. in [164] present a direct-touch device and a tangible device as alternatives to two conventional UI control elements aiming to facilitate manipulation of process variables. The conducted evaluation showed that even though traditional mouse- and keyboard-based UI controls 
outperformed by speed, the tangible concept was reported to enable a better understanding of the performed control actions. 


\section{Chapter 3}

\section{Method}

Multiple design paradigms exist that can be chosen for driving a design research process depending on the final goals and circumstances. For example, the activity-centered design focuses on the tasks and activities that need to be accomplished. The creator-centered design relies on the skills and wisdom of designers. The systems-centered design focuses on the components of a system. User-centered design (UCD) paradigm focuses on user needs and goals.

Being an industrial PhD researcher at ABB Corporate Research, I had to comply with the company's guidelines in questions related to research methodology. In the UX group, where I belonged to during my $\mathrm{PhD}$, the setup of the research projects is arranged according to the UCD paradigm. In relation to my research, this setup meant studying the users and the context before creating artifacts, also employing appropriate methods and tools, e.g. field studies and evaluation tests. The nature of my research is constructive, i.e. in my research, I imagine and build design artifacts and explain them. The ultimate orientation towards creating design artifacts makes my research connect to the root of the Research through Design paradigm (RtD), namely to the field research genre [146] due to the collaborative and applied nature of my projects. 
In this chapter, I will provide an introduction into the UCD paradigm, as well as describe the theoretical basics of RtD. I will also describe how UCD is practiced in the UX group of ABB Corporate Research. Finally, I will describe my research approach in detail and the nature and purpose of the developed design artifacts.

\subsection{User-Centered Design}

The concept of User-Centered Design (UCD) was introduced by Norman and Draper [174] who aimed to emphasize that a product should meet the needs of its users. The goal of UCD is to design solutions of high usability and of good user experience. According to Nielsen [169], to be usable, the product must consider five basic user-oriented dimensions:

1. Learnability: it should be easy to learn.

2. Efficiency: it should be efficient for the user's needs.

3. Memorability: it should be easy to recall how to use its functionality.

4. Errors: it should have a low error rate, the few occurring errors should be easy to recover from.

5. Satisfaction: it should be appealing to use.

Importantly, depending on the context and target users, the importance of these dimensions will differ [169]. The extent to which a product follows these dimensions will to a large extent define the user experience. The term user experience (UX) describes a person's emotions and attitudes about using a product. It was popularized by Norman, who generally advocated that no product is an island, but a cohesive, integrated set of experiences [173]. 
The core principles of UCD were formulated by Gould et al. [91, 90], who advocated that, in order to design usable systems, one should a) have an early focus on users, i.e. have direct contact with them, b) conduct empirical "hands-on" measurements, i.e. continuously test the users and evaluate the artifact, c) practice iterative design, i.e. the artifact must be iteratively modified based on the results of usability and performance evaluation results, d) practice integrated design, i.e. all relevant aspects of the design should be developed in parallel and under one management. Later, these principles became the foundation for the formulation of the ISO 13407 standard [116] that provides guidance for user-centered design processes for interactive systems. In addition to the aforementioned principles, the standard emphasizes the need to ensure appropriate function allocation between users and technology and the need for multidisciplinary design teams.

The first step towards the UCD process is to identify who is the actual user and decide how to involve them in the design process [2]. Eason [64] identified three types of users: primary, i.e. those who actually use the design artifact, secondary, i.e. those who occasionally use the artifact, and tertiary, i.e. those who might get affected by the usage of the artifact or who make decisions about purchasing it. A successful design of a product takes into account the wide range of stakeholders of the artifact [2].

In the literature, there is no common agreement on the particular flow of the UCD process, it is applied in a great variety of ways. However, based on the literature discovered, four major phases can be outlined, namely 1) understanding the user context, 2) specifying the user requirements, 3) designing solutions, 4) evaluating the solution against the original requirements and user needs. In each phase, there are a plethora of ways how to engage the user in the design process. Bevan provides a comprehensive overview of different methods suitable for different stages of the UCD process [21]; Vredenburg et al. [232] identified a set of 16 commonly used UCD methods together with their perceived key benefits and weaknesses. In general, with respect to 
the degree of the user involvement, UCD methods can be divided into three categories [131], namely a) design for users, i.e. the user is treated as an information source by means of questionnaires, interviews, and observations [94], b) design with users, i.e. where a formal dialog with users is maintained by means of conducting user evaluations and gather feedback, and c) design by users, where users are active participants, see, for example, the Scandinavian tradition of active user involvement often referred to as participatory design [197].

With respect to efficiency of UCD, in the literature, there is evidence that UCD methods make a significant impact on product development by improving the usefulness and usability of products [232]. On the other hand, many practitioners have considerations about increased expenses of UCD methods, e.g. usability testing is often considered as costly [232]. Another typical complaint about UCD is that it leads to unimaginative design due to the conservative bias of users engaged in the design process [229, 103].

\subsection{Research through Design}

Research through Design (RtD) paradigm centers around making. It was first introduced by Frayling [80], who argued that creating something ultimately new and communicating the process together with the result incorporates research as long as a) the report clearly states what is being achieved and b) communicates it through the activities of art and design. RtD suggests that knowledge is generated through the process of making, i.e. through describing and explaining the designed artifacts and documenting their creation process [146]. In [246], Zimmerman maps the stages of a traditional user-centered interaction design process with the opportunities to capture the knowledge they produce. Such produced knowledge is generative in its nature, i.e. it can inspire future design work [85]. One way of assessing generative knowledge is to analyze if it is evocative enough to originate new 
concepts and if it is open and inviting for others to join the discussion, also if it is framed in a way that is "open to multiple interpretations and reinterpretations" [106].

In the practice of RtD, designers explore new problem spaces communicating their understandings and visions through the construction of artifacts [248]. In the original work by Frayling, RtD denotes "research where the end product is an artefact - where the thinking is, so to speak, embodied in the artefact, where the goal is not primarily communicable knowledge in the sense of verbal communication, but in the sense of visual or iconic or imagistic communication" [80]. The artifacts provide concrete embodiments of a) the problem of the current state, b) the theory, c) the preferred future state, and d) technical opportunities which makes them an appropriate conduit for the transfer of research knowledge to the practice community [249, 248]. In the literature, artifacts are often considered knowledge per se as they answer the research question "How an object of interest can be designed?" [155].

$\mathrm{RtD}$ is essentially future-oriented, i.e. it does not aim to explain the current state of the world, but it attempts to understand it and imagine an improved future state [247, 146]. RtD stresses design artifacts as design exemplars that can transform the world from its today's state to the desired future [249].

Because the artifacts are the embodiments of possible futures, designers create them "through a process of disciplined imagination" rather than derive their ideas from the analysis of the current and the past situations [248]. Zimmerman and Forlizzi [248] categorized possible motivation of RtD work into two approaches, namely a) philosophical when researchers take a specific philosophical stance as a starting point and investigate and embody it through a process of making, and $b$ ) grounded when researchers focus on real-world problems by making artifacts that combine both a concrete framing of the problem and an expression of the desired future state. In both cases, designers 


\section{Method}

make propositions of a preferred future, i.e. "what could/should be", through the creation of artifacts. Koskinen et al. [146] highlights three approaches to RtD, namely Lab, Field, and Showroom. The lab approach assumes conducting research in the laboratory environment, i.e. taking the subject of the study from its natural environment and bringing it into a controlled environment where it can be subjected to experimentation. The field research genre of RtD assumes studying the users and the context before creating artifacts and employs methods and tools from design practice, participatory design and action research, e.g. field studies, user-centered design and evaluation tests. The showroom approach builds on exhibiting artifacts in the middle of theoretical frameworks which assumes the dominance of high-quality finishing of designs over theory and explanation.

$\mathrm{RtD}$ is argued to be a way to work with complicated design problems [248]. Even though the practice of RtD does not aim to produce products nor to have the intended effects in the domain/context in focus [248, 146], the artifacts and the knowledge generated through the process of their creation can be useful for the industry as inspiration for product development as the designs "can open up possibilities and prepare actions" [146].

On the other hand, RtD has been criticized due to its fuzzy definitions and lack of theory to guide practice [124]. With respect to developing theories, authors also point out the mismatch between the goal of theory, i.e. creating a unifying common ground, and the goal of design practice, i.e. creating particular examples [218]. Zimmerman and Forlizzi [248] argue that RtD outcomes can form the basis of design theories because a) they are specific exemplars of a theory, and $b$ ) they have extensible constructs and can describe relationships among groups of outcomes and phenomena. In the literature, artifacts and theory are often considered to be the endpoints of the same continuum of knowledge abstraction [155, 110], where artifacts are not abstracted constructs that are tightly connected to their intended context and might not even be meaningful outside it, while a theory is 
fully generalizable and holds under all circumstances. Artifacts can be complemented with the designer's comments, i.e. textual assessments and theoretical proclamations, which in the literature are referred to as annotations [86, 85]. Gaver and Bower argue that designs should be annotated if they are intended to bear clear and accountable knowledge contributions to research [85]. In the literature, annotations are considered as examples of theoretical statements residing in the inbetween space of the knowledge abstraction continuum as they add extra knowledge by pointing at qualities of artifacts, as well as issues of concern, that are important for research [155, 85]. When applied to a collection of artifacts, annotations make them into a portfolio by capturing family resemblances between them in terms of similarities and differences [85, 27]. Annotated portfolios are potential alternatives to formalized theory in conceptual development and practical guidance for design; grounded in the ability to elicit particular design spaces, reveal their dimensions, and uncover designers' visions, they have explanatory and predictive power [85, 27]. Importantly, annotations to a portfolio can be formulated in a variety of ways reflecting different intentions and targeting diverse audiences [85, 27].

\subsection{Research process at ABB Corporate Research}

In the UX group at $\mathrm{ABB}$ Corporate Research (CRC), the work is project-based. Projects can be either initiated by ABB or can be collaborative with third parties, such as industrial partners or universities. ABB-initiated projects are arranged according to the gate model. The model assumes that projects are divided into distinct phases separated by decision points, i.e. gates. Reaching a certain gate means having a meeting with project stakeholders where the results of the projects are being presented and high-level plans for the next project stage are being defined. Also, at each gate, the stakeholders are deciding whether 


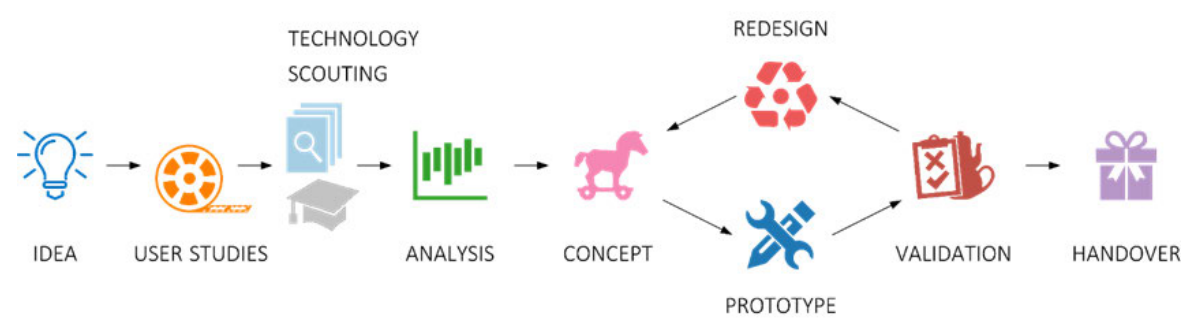

Figure 3.1: The user-centered design process practiced at ABB CRC, UX group. Source: ABB.

the project will be continued, suspended or canceled. The latter can happen, for example, if the stakeholders find the results insufficient or the project is losing its relevance for the company, also if there are no sufficient resources in the project or there is no agreement on how to proceed, etc. The stakeholders are usually representatives of a business unit at $\mathrm{ABB}$ who have initiated the project and who will take over the project results. Generally, they represent $\mathrm{ABB}^{\prime}$ s business interests in the project; for example, they are interested that project results are relevant to ABB's products and customers and can be potentially productized.

Collaborative projects with third parties usually do not run according to the gate model. Instead, there is a steering committee where the representatives of each party speak for the interests of their company. The frequency of meetings and reporting is defined based on a common agreement of the parties, as well as taking into account the requirements of the co-funding organizations. ABB representatives are usually managers from business units that find the potential results from the collaboration relevant to their current or future products. Stakeholders have all the power to influence the orientation of the project and its outcomes.

During a calendar year, multiple projects are ongoing in parallel. Usually, several researchers are involved into a project, one researcher can be involved in several projects simultaneously. In the UX group, the 


\subsection{Research process at ABB Corporate Research}

researchers are practicing a custom version of the UCD paradigm. On various stages of the UCD process, based on personal preferences or project demands, some of the participants could be involved in the project activities more or less actively. For example, some researchers might be more keen to participate in field studies whereas others are more excited about analysis and brainstorming around the collected materials. It is not exceptional that, while the project is ongoing, new participants are being assigned to the project or leave the project in response to changing demands or workloads.

The high-level direction of research and the context of the project, i.e. the particular industrial domain, are usually defined by business stakeholders or upper management who initiated the project. In rare cases, smaller scale projects, the so-called pre-studies, could also be proposed by the researchers themselves. A typical ultimate goal of a project is to increase the efficiency of industrial workflows in the domain in focus, improving the user experience per se is rarely the goal of a project.

The research projects consist of multiple stages inline with the customized process of the UCD paradigm, see Figure 3.1. A project usually starts with a vague idea of what should be achieved in its scope. The project continues with field studies. The field studies incorporate visiting industrial settings, interviewing, and observing relevant personnel. The goal of conducting field studies is to build background knowledge about the industrial domain and get familiar with the routines, problems, and needs of the industrial personnel of interest. The broad network of ABB customers opens up the potential to get access to various kinds of industrial domains and workers of different roles. However, in reality, it is effortful to actually engage with industrial workers due to their busy schedules. Having a short visit to an industrial field including a couple of hours of quality time with industrial workers is the reality of such field studies. 


\section{Method}

The materials collected during field studies are ordinarily analyzed using methods of Contextual Design [109], e.g. the affinity diagram. The goal of the analysis is to structure a large quantity of collected information and outline major problems and challenges related to the work practices which could be potentially improved using new approaches and modern technologies.

Once the problems are outlined, the project proceeds to the background search and technology scouting. In these stages, the intention is to judge what was done in the field before and what can be achieved today using the available technology. Usually, the researchers utilize available on the market state-of-the-art technology, i.e. technological devices and techniques that employ the most current and high-level IT developments. What is usually looked for is the technology that is robust enough to be used in high-fidelity prototyping but at the same time represents the frontiers of knowledge. For example, in various projects, me and my ABB colleagues used such novel off-the-shelf devices as Kinect, Tobii, Leap Motion, Oculus Rift, etc.

The project proceeds to the ideation phase. Its goal is to look for inspiration and ideas. The researchers use mostly sketching on paper to express and discuss ideas. In addition, the process can be facilitated by arranging interactive workshops with industrial personnel and stakeholders. During these participatory design sessions, the goal is to actively engage the users in creating new and exploring existing design ideas. Often, the ideas are discussed with the business stakeholders before getting implemented. Indeed, pragmatic and product-oriented ideas get the most support; an extra effort is usually needed to get the green light for working on non-conventional ideas.

The next phase of the project is iterative and incorporates low and high fidelity prototyping. The involved activities on this project stage consist of paper prototyping, sketching in graphical editors and sketching in code. During the prototyping stage, preliminary user evaluation sessions might be arranged. During these sessions, the devel- 
oped prototypes are demonstrated and explained to the users to get their feedback and opinions. Furthermore, workshops might be arranged with university representatives where developed concepts are demonstrated and discussed from the novelty perspective. The received comments might be considered in the next development iterations. Preliminary demonstrations to stakeholders are also likely to be included in the project flow.

The project then proceeds to a validation phase. In this phase, the researchers take the developed solutions and go back to the field where they can demonstrate the solutions to the real user and collect their feedback. In the UX group, mostly qualitative evaluation is practiced. This means that technologies are demonstrated to the potential uses and their feedback is collected. Depending on the fidelity of the prototype, the users might try to use the solution in a short- or long-term user test. Observations and feedback are then processed and analyzed. However, due to the problems described earlier in this chapter related to the access to industrial workers, validation in real settings might be complicated. Furthermore, if the project has only low fidelity prototypes, such as sketches, the feedback can be only verbal, a sort of a discussion.

The final stage of the project is the handover of the results to the business unit. All the product-related knowledge and solutions are collected, documented and handed over to the receiving business unit at $\mathrm{ABB}$. The future of the project results is usually obscure to the researchers. Stakeholders not necessarily give feedback whether the results of the project will be productized or abandoned.

\subsection{My research practices}

In my $\mathrm{PhD}$ research, I was relying on the UCD process practiced in the UX group at ABB CRC. However, because it did not strictly define concrete methods on each stage, nor the order of the stages or 
the required information flow between the stages, I had some freedom to adjust the process for the benefits of my research. In my case, relying on the UCD paradigm implied that the starting point of my research was always industrial context, industrial users and their problems. In the scope of my research, I have visited many industrial settings, mostly factories and their control rooms. During the visits, I was observing and interviewing industrial workers, focusing on their tools and routines. However, unlike my colleagues in the UX group, I treated the knowledge gained from the field and industrial users more as a source of inspiration rather than as a detailed prescription of what to do. During my visits, I tried not to pay too much attention to concrete complaints of particular industrial workers because, most likely, they would reflect their personal discomfort in daily workflows. Solving such problems would mean doing consultancy, i.e. improving the tools of one particular industrial company active in a certain industrial domain. Instead, I tried to think critically about the knowledge gained during the field studies. I aimed to see the overall picture, i.e. bottlenecks, typical routines, trends in the development of industrial control rooms across different industrial domains.

With respect to field study data processing, I preferred to go through the collected data informally, individually and at my own pace treating it as a source of spontaneous inspiration rather than a handbook for thorough reading. I was not a big adherent of conducting formal activities of field studies analysis practiced in the UX group i.e. personas and affinity diagram. First of all, to be done thoroughly, these activities require a lot of time which is usually a limited resource in the project. Second, due to too much emphasis on the comments of field workers, they provide a very pragmatic view on the problems in the field as they are mainly a reflection of the problems experienced by the interviewed workers. Finally, I am not fully convinced that the affinity diagram or similar data structuring methods are the silver bullet in handling unstructured field material. The resulting clusters are only one possible way of organizing the data out of a myriad of other 
possibilities. Every quote or observation can be interpreted in a number of different ways creating a multitude of possible versions of the affinity diagram. On the other hand, I could understand the importance of these standard analysis approaches for ABB. Relying on the materials from the interviews can motivate concepts to the business stakeholders and thus ensure the continuation of projects. Therefore, I could clearly see why my colleagues were practicing the standard analysis means. Furthermore, I have often considered the assumptions and discoveries originated from their analysis in my work, but again more as a source of inspiration rather than guidance to action.

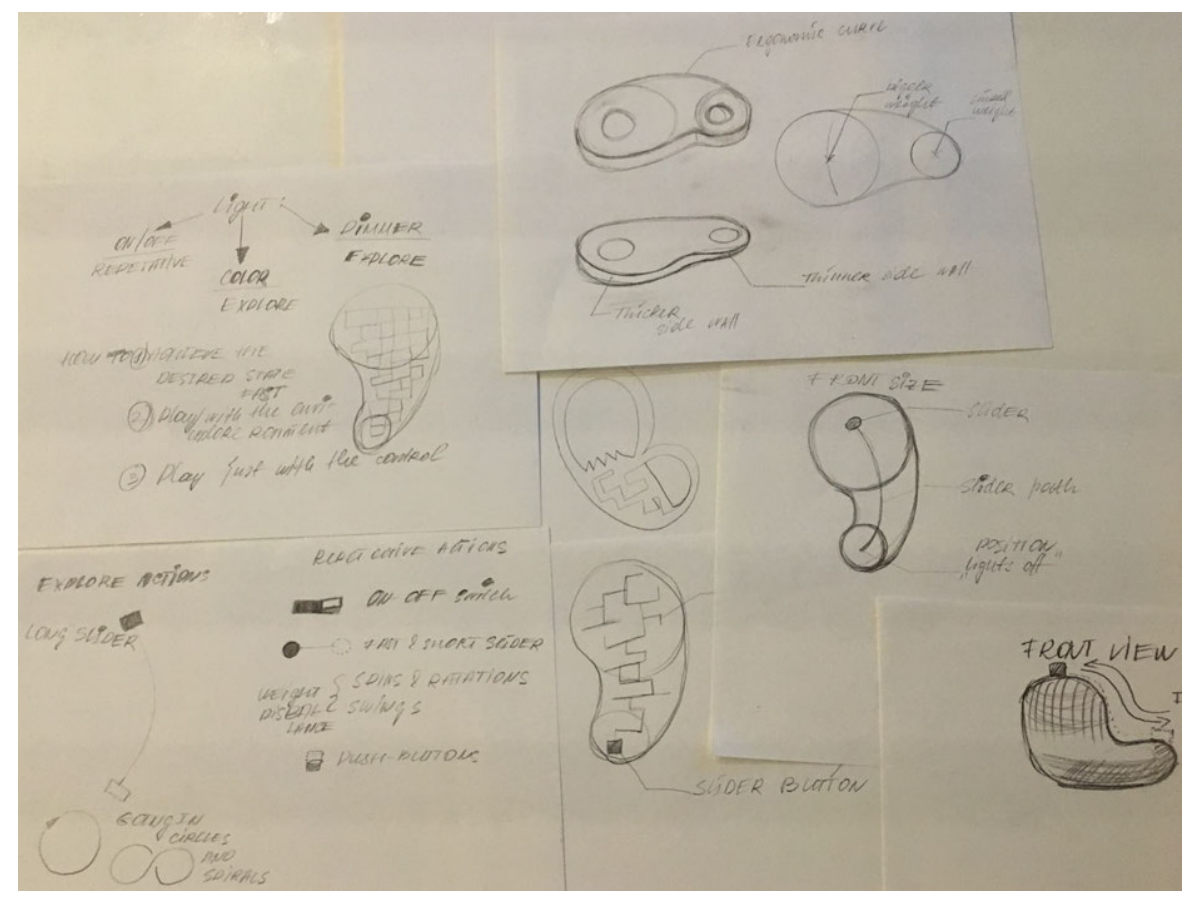

Figure 3.2: Sketching on paper: exploring the idea of a hand-held fidgeting device that could be used for controlling the surrounding environment.

The ideation phase of the UCD paradigm is one of my favorite project stages. I enjoy generating and developing ideas. In particular, I like looking for unexpected and unanticipated solutions. I find inspiration 


\section{Method}

in art, history, cognitive sciences, and technology. To express and develop my ideas, I primarily use sketching on paper, see Figure 3.2. I find this approach easy, cheap and, most importantly, fast and flexible. When an idea forms to a decent level, I perform sketching in graphical editors, such as InkScape ${ }^{1}$ - a free tool allowing to create images in vector graphics, to fine-grain the idea and prepare it for a presentation. My sketches are static even though the ideas often contained some kind of motion. I believe that my sketches could benefit from using animation, especially when there was a need to demonstrate them to outsiders, but I did not have enough skills to create animated solutions in a fast manner nor time to develop such skills. To compensate this omission, I was keen on moving to prototyping and sketching in code.

Prototyping is my next favorite stage of the research process. I like creating high-fidelity dynamic solutions that can be tested and evaluated by a test user and demonstrated in action to a public. In line with some design practitioners, I believe that design research aiming to produce knowledge concerning design cannot address research questions without doing some kind of design practice. In my case, designing and developing prototypes is my design practice. The value of prototypes in my research has a dual nature. First, a prototype serves as a potential product example for the business stakeholders. Second, in line with the vision of Zimmerman et al. [249], I treat my prototypes as embodiment of design theory and knowledge that can be argued to constitute contributions to the HCI discipline; they externalize conceptual ideas that I come up with in the scope of my research projects.

In my research practice, I have developed low- and high- fidelity prototypes. With respect to low-fidelity prototyping, when working on tangible devices, I used paper and foam board, see Figure 3.3. However, when working on digital solutions, I rarely used low-fidelity prototyping, instead I was widely relying on sketching in code. In my eyes, sketching in code is the easiest way to experiment with the

${ }^{1}$ https://inkscape.org/ 


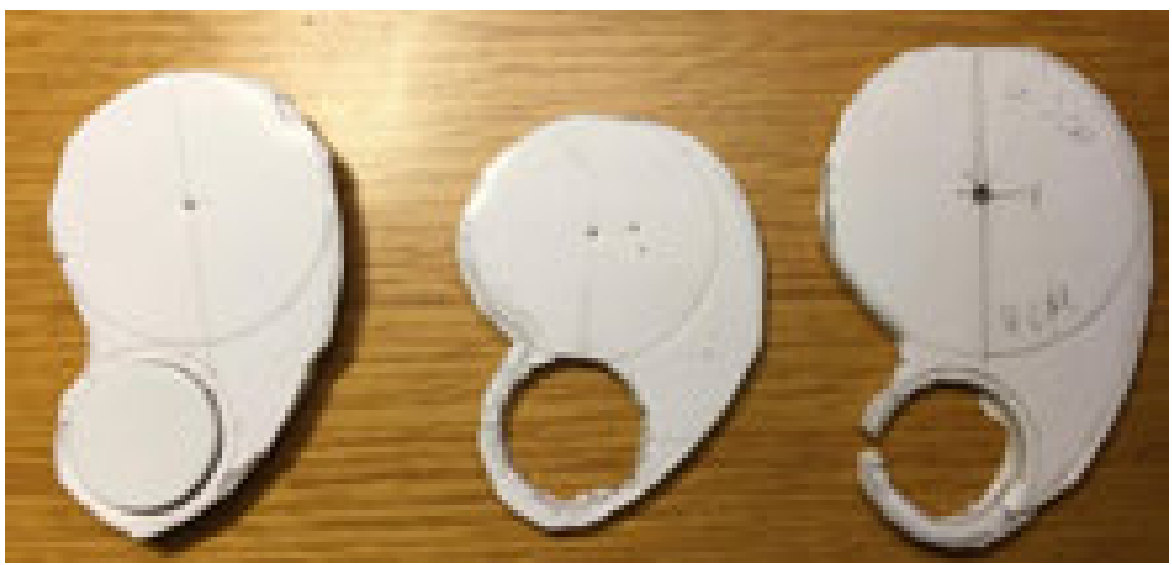

Figure 3.3: Low-fidelity prototypes from foam board: experimenting with different shapes of a hand-held fidgeting device.

functionality and look-and-feel of the solution. Furthermore, as practice showed, stakeholders and field workers receive sketches in code much better than paper concepts. This is arguably due to the fact that for representatives outside of the design community it may be hard to comprehend the implied dynamic experiences and temporal properties of the concepts that are depicted as static sketches [156]. The prototypes that I developed comply with the so-called fundamental prototyping principle [153]. Namely, they are developed to a manifestation that filters the qualities which are interesting from the research perspective without distorting the overall understanding of the concept. Due to this reason, my prototypes usually lacked functionality that would be essential for a finalized product but was not important for the scope of the research project. Moreover, I rarely added ABB's branding or applied their style guidelines in my designs.

For the prototyping of data visualizations, I was relying on existing Web technologies such as JavaScript, Node.js ${ }^{2}$. HTML, CSS and SVG. Occasionally, I was relying on third-party libraries to speed up the

\footnotetext{
${ }^{2}$ https://nodejs.org/
} 
coding process, e.g. D3.js $2^{3}$ which is a free library that simplifies working with SVG graphics and data visualization, as well as with animation. For desktop solutions, I usually relied on .NET technologies, namely CSharp and WPF. This is mostly because ABB is widely relying on Microsoft products and technologies. For physical prototypes, I used CAD-software for 3D modeling and a 3D-printer at ABB CRC for printing the casing for the high-fidelity solutions. With regard to electronics, I relied on Arduind 4 -like micro-controllers that allow easy and rapid programming.

The user evaluation stage was very important for the flow of my research. I tended to evaluate my solutions with real users whenever possible. However, due to the reasons explained in the previous chapter, it was an arduous task to involve industrial workers in the evaluation of the prototypes. In the best case, I could engage one or two industrial workers as test users in a short session of evaluation. I primarily used qualitative evaluation, i.e. I was demonstrating the solution in action to the users and asking for their feedback. Alternatively, if the solution was stable and well-elaborated, the users could try the solution themselves and report their thoughts and impressions. In one of my last projects, I have conducted lab tests with several dozens of non-industrial users as participants; I collected both the numerical data of their performance and qualitative data of their personal impressions. The drawback of involving non-industrial users is that it is hard to generalize whether the performance and preferences of actual industrial workers will be similar to the test participants. There is no doubt that the behavior of actual industrial workers is likely to differ from the test users due to their knowledge, professional experience, and training. However, in order to test the performance of actual industrial workers, one needs to implement test cases on their interfaces which assumes a) access to the interfaces and b) integrating the test solution into the actual interfaces, which will require professional

\footnotetext{
${ }^{3}$ https://d3js.org/

${ }^{4}$ https://www.arduino.cc/
} 
knowledge about the control system. Taking into account the amount of needed effort, it is not practical to directly approach industrial users to test a very fresh idea. This is when experiments with test users come in handy. They will show whether the initial project assumptions are valid. If the answer is yes, the test user results can be used to motivate the continuation of the research project and request access to the real workers and their interfaces. 



\section{Chapter 4}

\section{Conducted work}

In the scope of my $\mathrm{PhD}$ research, I have participated in a variety of diverse projects. Most of them were initiated and run at $A B B$, several were university projects. In my research practice, I often could choose the projects to participate in. I normally preferred those that were closer to the research questions of my $\mathrm{PhD}$, i.e. had some relation to industrial control rooms or data visualization. Below, I list all the projects, briefly describe their goals, my research activities, and main research outcomes with regard to my $\mathrm{PhD}$.

1. Tangible interaction (2015): This short-term pre-study project was initiated and run solely by ABB CRC UX group. Its goal was to explore the design space of potential tangible interfaces for industrial control rooms. The topic of the project was perfectly in line with the research interests of my PhD. In the project, I was working on one commonly defined idea, namely the shift report button, and one concept of my own, i.e. the tactile mouse. The work resulted in one academic publication "Re-Introducing Physical User Interfaces into Industrial Control Rooms" [56], see Paper 8 in the appendix.

2. Future Operator Interaction(2015-2016): This long-term project was initiated and run solely by ABB CRC UX group in 
tight collaboration with one ABB Business Unit working with process control. The goal of this project was to explore the possibilities of operator interaction in the industrial control rooms of the next generation. The main focus was dedicated to targeted alarm delivery and to alternative interaction means with the control station. In the scope of the project, me and several colleagues from the UX group were working on novel ideas, whereas I was implementing them in practice using a simplified replica of a control system. The main outcome of this work became the interactive zones concept. The work resulted in one academic publication "Untethered Workspaces: A Zones Concept Towards Supporting Operator Movements in Control Rooms" [52], see Paper 11 in the appendix.

3. Intelligent Alarm Management(2016): This long-term project was initiated and run collaboratively between several ABB CRC groups. The goal of this project was to improve the efficiency of industrial alarm systems by managing alarms in an intelligent way. The idea was to extract alarm sequences from the alarm log of a factory and visualize the results so that they could be analyzed. The gained knowledge could be used to create a more efficient configuration of the industrial alarm system so that only important alarms will be delivered to the industrial control room and non-informative alarms will be suppressed. My role in this project was to design the visualizations of alarm sequences and develop an interface for the visual analytics. The main outcome of this work became the intelligent alarm management web portal. The work was described in one academic publication "Towards intelligent alarm management in the Age of IIoT" [53], see Paper 9 in the appendix. The gained knowledge related to sequential data visualization contributed to another academic publication "A Model for Types and Levels of Automation in Visual Analytics: Examples from Event-Sequence Analytics" [60], see Paper 3 in the appendix. 
4. Remote Expert 4.0 (2016-2017): This long-term project was initiated and run collaboratively by two ABB CRC groups also in tight collaboration with one ABB Business Unit working with marine services. The goal of this project was to design and develop novel tools for supporting remote service engineers working mainly in the marine domain. The project was very applied leaving not much space for the ideation. The goal was to aggregate the variety of tools used by remote experts into a common system with a single entry-point and an overview of the current work situation. The main result in this work became the marine web portal. The work resulted in one academic publication "Supporting maritime remote experts working over distance" [250], see Paper 10 in the appendix. As a shortterm side activity of this project, I became engaged in development of a data visualization for remote service engineers working in the industrial robotics domain. The goal was to design and develop a novel information visualization that would let the operators obtain better situational awareness over the status of a large fleet of distributed robots. The main result of this work became the overheated robots visualization. The work resulted in one academic publication "Towards Effective Industrial Robot Fleet Visualization for Remote Service Applications" [61], see Paper 7 in the appendix.

5. Visualization for Sustainable City (2018-2019): This longterm project was initiated and run collaboratively between multiple stakeholders, including ABB, several industrial factories, and RISE. The goal of this project was to look into innovative information visualizations for the district heating domain that would improve industrial operators' understanding of the status of the district heating system of a city. The original expectation from the project was to design conventional solutions that could be directly employed by the industrial stakeholders. However, in the flow of the project, it was decided to look for alternative visualization means. This initiative led 


\section{CONDUCTED WORK}

to working on conveying the physicality of district heating processes and conveying the feeling of water by pure visual means. In this project, I designed and developed two visualization concepts, namely Medusas and Animated Pipelines. The work resulted in one academic publication "Feel the Water: Expressing Physicality of District Heating Processes in Functional Overview Displays" [55], see Paper 6 in the appendix.

6. AutoMed (2018-2019): This long-term project was initiated and run collaboratively between multiple stakeholders, including ABB, several municipal hospitals, and RISE. The goal of this project was to increase the efficiency of Swedish municipal hospitals using the data-driven approach. However, the quality of the data turned out to be too poor to achieve the original goals. Instead, the focus of the project shifted towards implementing a Visual Analytics system that could help understand and pre-process the data and manually identify bottlenecks in patient flows and resource allocation. Even though the medical domain is far from my competence or interest, the generic nature of the topic, i.e. effective visualization of large amounts of data, was a good match with the research goals of my $\mathrm{PhD}$. In this project, I was fully engaged in working on the data visualization solution, i.e. the AutoMed web portal, which became the main outcome of the project. The work resulted in one academic publication "Visualization for quality health-care: patient flow exploration" [57], see Paper 5 in the appendix.

7. Sustainable Underground Mining (2019): This long-term project was initiated and run collaboratively between multiple stakeholders, including ABB, several ind ustrial mining companies, and Combitech. The ambitious goal of this project was to create a vision of the Swedish underground mine of 2025 and develop prototypes of future tools and solutions that would support the vision. Misunderstandings between the stakehold- 
ers and the ambiguity of the original goals did not add productivity to the research processes. Often the project was put on hold due to the absence of concrete ideas. I primarily used my time in the project for working on my ideas on guiding the operator's attention and improving their situation awareness. The main outcome of this work became two high-fidelity prototypes of visual aid systems capable to guide the operator's attention to the most urgent information. The work resulted in one academic publication "Guiding the Operator's Attention Among a Plurality of Operator Workstation Screens" [51], see Paper 2 in the appendix.

Two projects were part of university courses. In this case, I had more freedom and could shape the topic of the project and its scope myself. However, I had to take into account requirements of the course and interests of other participants if any.

1. Human-Automation Collaboration course (2017): The purpose of the course was to enhance understanding of how to shape human-automation collaboration through theoretical foundations and concepts from cognitive science, automation taxonomies, interaction design, and other related fields. The course incorporated an independent project where one was supposed to contextualize the theoretical foundations by conducting small-scale research on a relevant topic. In this project, I was working on the topic of interacting with office automation. The main outcome of my work became the fidgeting device. The work resulted in one academic publication "Fidgeting with the Environment: a Tangible Control for Interacting with a Smart Light" [50], see Paper 1 in the appendix.

2. WASP Research Arena Public Safety project (2019): This project was initiated by WASP research arena for public safety, 
i.e. WARA-PS ${ }^{1}$ WARA-PS provides a realistic demonstration environment for scenarios that focus on rescuing on water. The idea of the project was to elaborate an existing control system, i.e. the WARA-PS demonstrator, that allows monitoring the situation on the water in a geographic area and controlling the rescue fleet of autonomous and manned agents. My role in the project was to improve the look-and-feel of its interface. The main outcome of this work became the customizable dashboard of WARA-PS demonstrator. The work resulted in one academic publication "Improving Usability of Decision Support Systems for SAR Operations: WARA-PS case study" [54], see Paper 4 in the appendix.

The collection of diverse artifacts, that are results of participation in the described projects, can be roughly divided into two major groups, namely data visualizations and alternative interaction means. Further in this chapter, I describe each of the developed prototypes in detail.

\subsection{Data visualization}

In the course of my research, I have worked with industrial visualizations of various types and purposes. Two major groups can be outlined: overview screens for real-time process monitoring and interfaces for Visual Analytics. In both cases, I was investigating potential ways of how cognitive and visual loads of the interfaces could be reduced while keeping the information presentation compact, informative, visually appealing and straightforward for interaction. In addition, I was trying to move away from the standard industrial data visualization approaches towards non-trivial but still valid, i.e. informative and functional, alternatives.

\footnotetext{
${ }^{1}$ https://wasp-sweden.org/demonstrators/wara-ps-publicsafety /
} 


\section{Overview screens}

When designing overview screens, I was either following the standard approach of creating a conventional dashboard or going for an alternative visualization. I preferred dashboards when working in applied projects that were restricted in time and did not provide much space for ideation. I went for an alternative visualization when project setup encouraged creative thinking. In this section, I will discuss the examples of both conventional and alternative overview screens developed in the scope of my PhD.

\section{Dashboards}

In the course of my research, I have designed and developed two conventional dashboards, namely the marine web-portal [250], see Figure 4.1. and the interactive dashboard of the WARA-PS demonstrator [54], see Figure 4.3 .

The goal of the project in the marine domain was to aggregate a collection of stand-alone tools used by marine operators in their daily routines into one common system that would provide a single start point for all the operator's workflows. The work resulted in a web portal with a dashboard as a starting page and a set of integrated tools available on-demand as separate system views.

The goal of the WARA-PS project was to improve the look-and-feel of the existing WARA-PS control system. The original interface, see 4.2 , had multiple problems. First of all, the interface did not provide the user with an instant overview of the system status. To get a decent SA, the user would need to click through the tabs in the menu in the right corner of the interface. Second, the map taking the central place in the interface was impractical mostly because it is hard to compare and prioritize one mission over another using a map whereas prioritizing is one of the critical tasks in SAR operators' work [101, 184]. Therefore, it was decided to improve the interface so that it would: 1) give 


\section{CONDUCTED WORK}

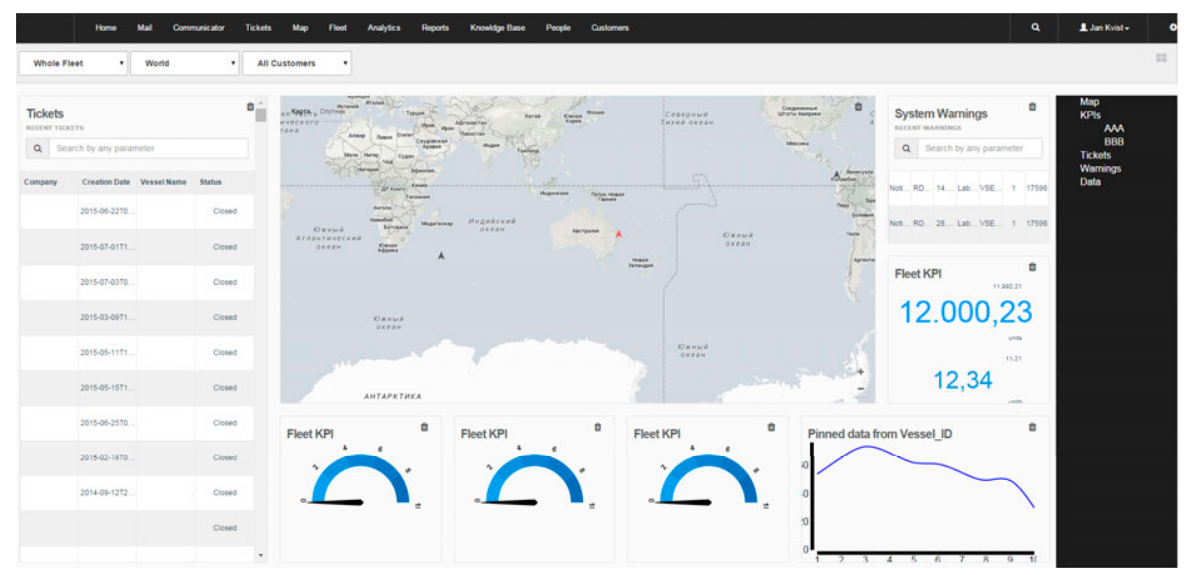

Figure 4.1: The main page of the marine portal. The look-and-feel of the solution is a conventional dashboard with self-service functionality.

a comprehensive overview of the system status while providing details on demand, 2) allow comparing reported disasters and ongoing missions, and 3) allow prioritizing agents. The final solution became a dashboard which aggregated and presented all the information that was initially available in the original interface, but in a compact way allowing to observe it all at once.

Even though the projects were taking place in different industrial domains, i.e. the marine domain and the public safety domain, the requirements had some similarities. For example, one of the main problems in both domains was too many diverse tools that the operators were using on a daily basis. For this reason, the developed solutions also became to some extent similar. Both resulting interfaces were implemented as a conventional dashboard with self-service features so that the presented content could be customized. The customization allowed the user to personalize the dashboard by deciding what information should be shown on the interface, also the size of the containers and their order. For the marine domain, the customization features were intended to satisfy the needs of operators of different roles who 


\subsection{Data visualization}

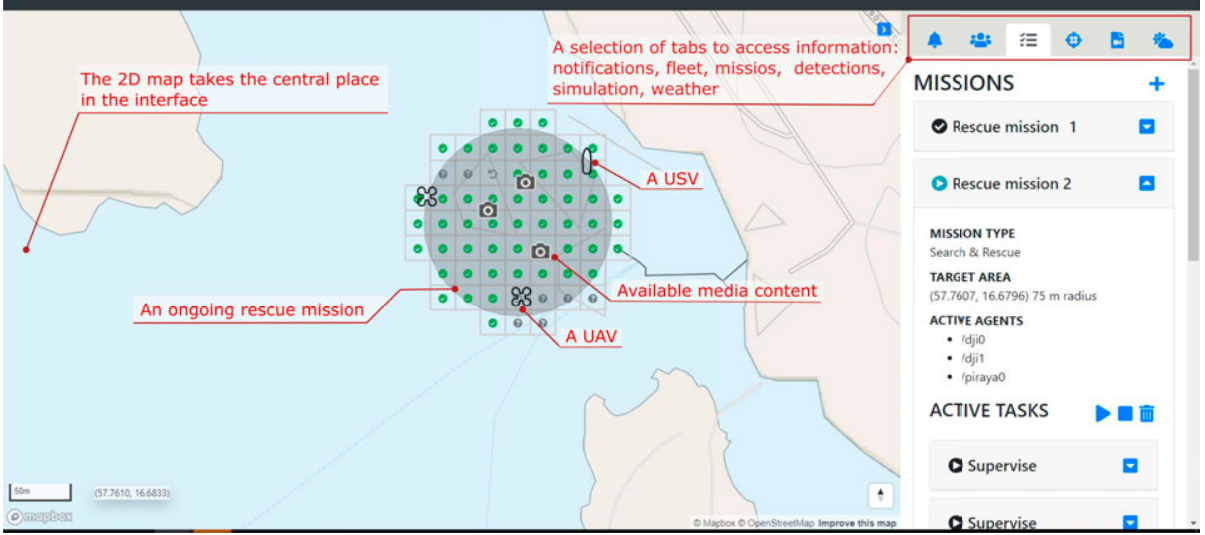

Figure 4.2: The original interface of the WARA-PS control system.

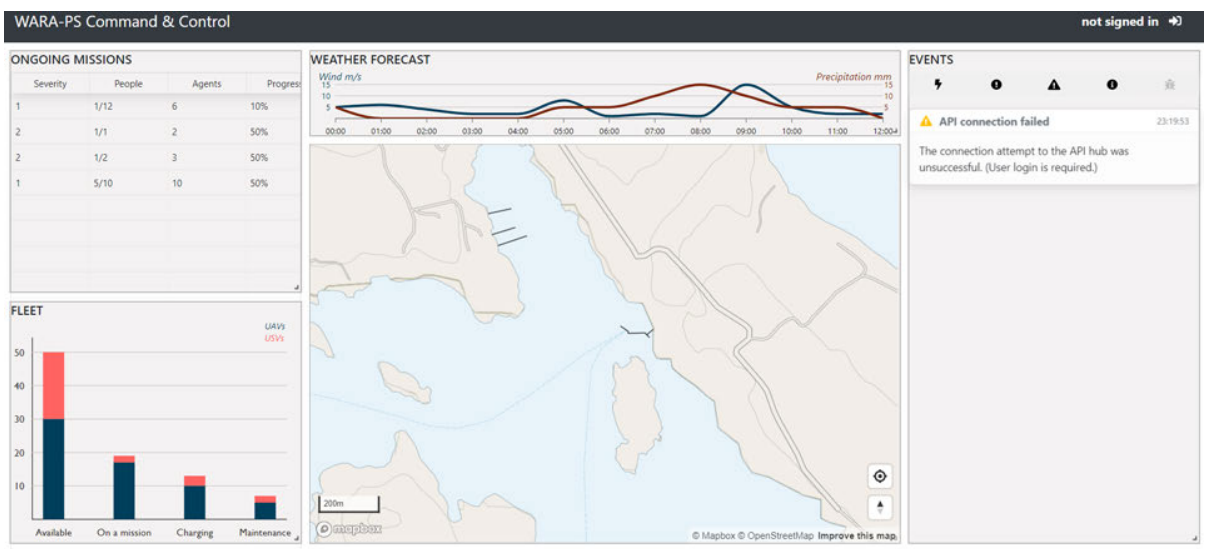

Figure 4.3: The customizable dashboard of the WARA-PS demonstrator. 


\section{CONDUCTED WORK}

needed to access different information depending on their role. For the public safety domain, the customization was intended to help the operators to adjust the dashboard to the different stages and different types of the missions. Furthermore, enabling the user to configure the interface themselves partially took the burden off my shoulders to define the information to be shown on the interfaces. Moreover, the developed dashboards not only serve as the overview displays but also function as an interactive filter and a search engine. For example, in the WARA-PS dashboard, when the user hovers a mission in Ongoing missions tile, other tiles adjust and show only relevant information related to the selected mission.

From the design perspective, both interfaces were not particularly novel. They employ rather conventional information visualization means of low graphical abstraction levels [31], i.e. tables that are convenient for comparing and sorting information, charts that are effective to present numerical data visually, and maps that were the key element of many of the original tools. The clear practical advantage of the developed dashboards is in the ability to consolidate various system views on one display, which helps to a) ensure a decent SA with minimal manual effort and b) customize the presented information according to the needs at hand. The main challenge in both projects was more in the technical implementation rather than in the design. Designing the dashboard layout in both cases was rather straightforward, but implementing the flexibility was not a trivial task.

\section{Alternative visualizations}

In the scope of my $\mathrm{PhD}$ research, I have developed three alternative information visualization interfaces, namely Overheated Robots [61], see Figure 4.4, and two interfaces for the district heating domain [55], i.e. Medusas, see Figure 4.6, and Animated Pipelines, see Figure 4.5. When working on their designs, I was 1) trying to avoid the conventional dashboard-like view, i.e. I was preferring a holistic picture to 
dividing the screen into discrete areas, and 2) aiming to design visual representations and layouts new to the industrial domain.

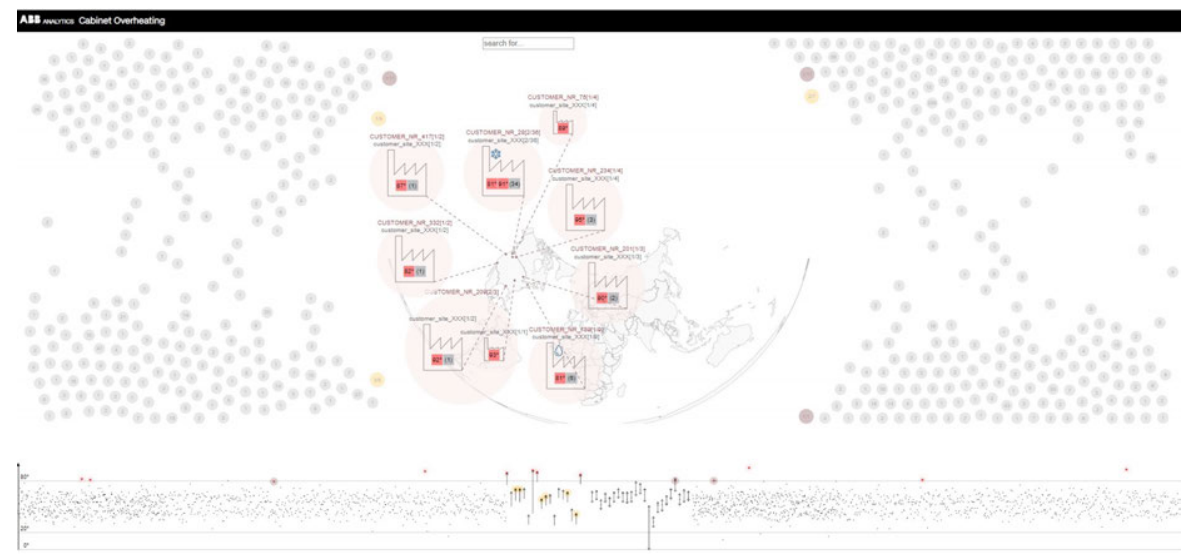

Figure 4.4: The Overheated Robots visualization demonstrates a combination of several alternative strategies for visualizing and laying out the fleet data.

The Overheated Robots interface, see Figure 4.4. visualizes a large fleet of robots using a collection of circles of different sizes, colors, and locations. The properties of circles are mapped to the properties of the particular fleet assets they represent. The interface demonstrates how a custom-made, visually pleasant, and ergonomically adequate visualization can compete in informativeness with conventional data visualizations such as tables or dashboards. The developed interface is interesting from at least two perspectives. First of all, due to hierarchical data grouping, i.e. arranging fleet items by their physical location and the customer, the interface is capable of visualizing thousands of fleet assets on a single view. Second, in this interface, I differentiate between the important and less relevant information and visually separate them. Visual encodings of urgent information, i.e. alarming assets, are depicted in much detail and of a larger size and are located in the central area of the interface. Visual encodings of secondary information, i.e. normally functioning fleet items, are depicted in a smaller size and in less detail, residing in the periphery of the view. 


\section{CONDUCTED WORK}

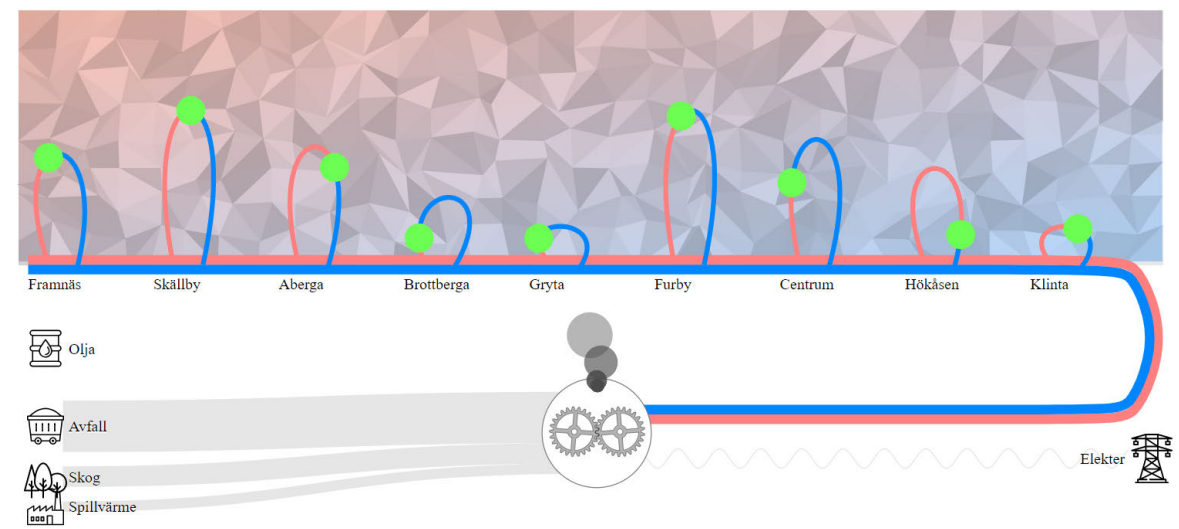

Figure 4.5: A screenshot of the Animated Pipelines visualization.

The two other visualizations, namely Medusas and Animated Pipelines, aim to visualize a district heating system of a city. Interestingly, they both depict the same data, but their approach to visualization is rather different. The Animated Pipelines interface, see Figure 4.5. aims to depict the entire district system in a rather simplified way. It reflects the basic components of the district heating system, i.e. production, delivery and consumption, and conveys the most important technical characteristics of the district heating network, i.e. pressure and temperature. The numerical KPIs are presented in a purely visual form allowing the interface to be free from numbers. For example, the delivery process is represented by red and blue pipes symbolizing hot and cold water flows respectively. The delivery end-points, i.e. residential areas, are presented as curves. The shape of a curve represents the consumption level, i.e. the height of a curve shows the amount of delivered energy and the color ratio depicts the balance between the delivered and the consumed energy. The animation of the curves reinforces the effect of the water being pumped. The animated background, behind the curves, depicts the weather conditions: the colder the area, the bluer is the background. 


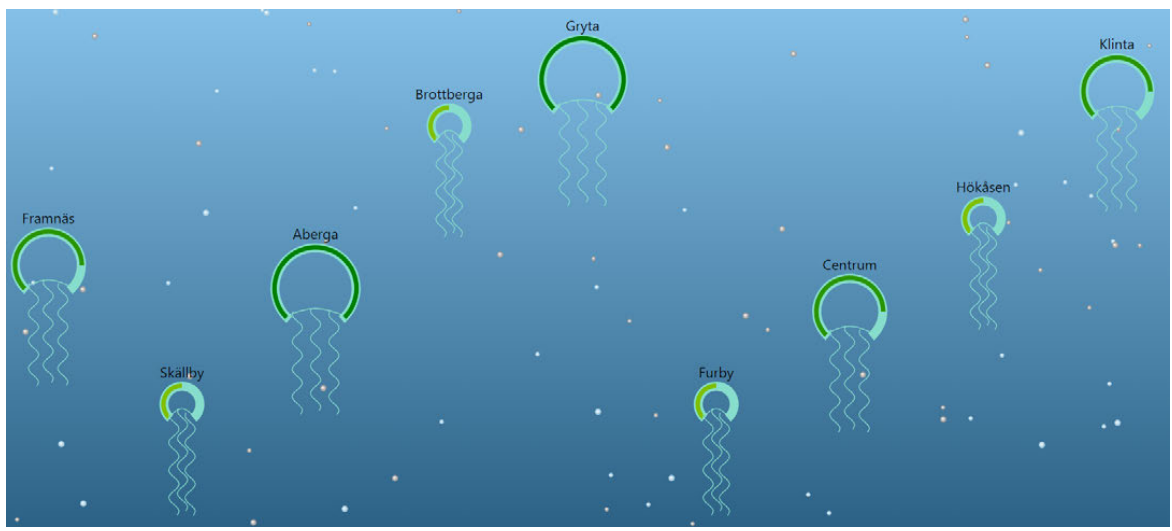

Figure 4.6: A screenshot of the Medusas interface.

The Medusas interface, see Figure 4.6, is more abstract in the sense that it does not attempt to depict the realistic setup of the district heating system. Furthermore, it uses a very non-industrial metaphor for depicting the status of the system, namely a jellyfish, i.e. a medusa. This metaphor was selected based on the fact that the pumping action a jellyfish uses to propel itself through the water is in a way similar to the workflow of a pump station supplying an area of a city with hot water. The designed visual shape of the medusa consists of a roundish head and a set of tentacles. The pulsating animation of the medusa's head aims to imitate the pumping action of a jellyfish and is a reference to how water is being pumped by the pump. The tentacles are also animated to create a feeling that the medusas are floating in the water. The vertical position of the jellyfish corresponds to the hot water consumption level in the area, i.e. the higher it is located in the interface the higher is the consumption level. The size of the medusa's head corresponds to the incoming hot water flow. The balance between the hot water consumption and delivery in an area can be derived by visually comparing the position of the medusa with the size of its head, i.e. the higher the position the larger the head should be. The gauge inside the head shows the current performance of the pump versus its overall capacity. The blue background of the interface symbolizes 


\section{CONDUCTED WORK}

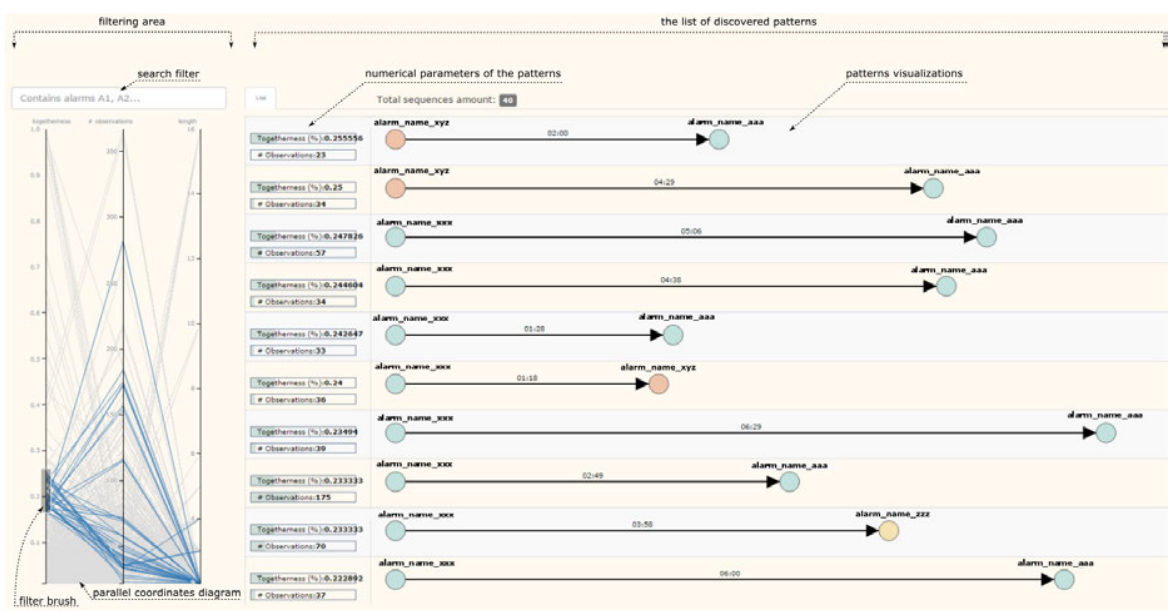

Figure 4.7: A screenshot of one of the views of the intelligent alarm management web portal.

water, small animated bubbles slowly raising upwards reinforce the feeling.

All the three interfaces, even though visually rather different, have many commonalities. To start with, they all try to give a comprehensive but compact overview of the industrial system that can fit on one screen. Next, all of them tend to avoid numerical and textual data presentations. Furthermore, they employ custom layouts that are holistic and do not divide the screen into areas like conventional dashboards would do. Moreover, they all use non-conventional visual data presentations of high levels of graphical abstraction [31] that are visually compact but still informative. Finally, in the last two interfaces, the visual markers not only show the status of the production but also reflect the physicality and technical specifics of the underlying industrial processes, e.g. they convey the feeling of water. 


\section{Visual Analytics}

I worked on Visual Analytics (VA) solutions in two projects, which resulted in two tools, namely the Intelligent Alarm Management web portal [53], see Figure 4.7, and the AutoMed web portal [57], see Figures 4.8 and 4.9 . The two systems were developed for different target domains, i.e. medical and industrial, therefore, they look different and target diverse use cases, furthermore, they aim to facilitate analyst's workflows on different stages of the VA process. Regarding commonalities, both of them touch upon the topic of the sequential data presentation.

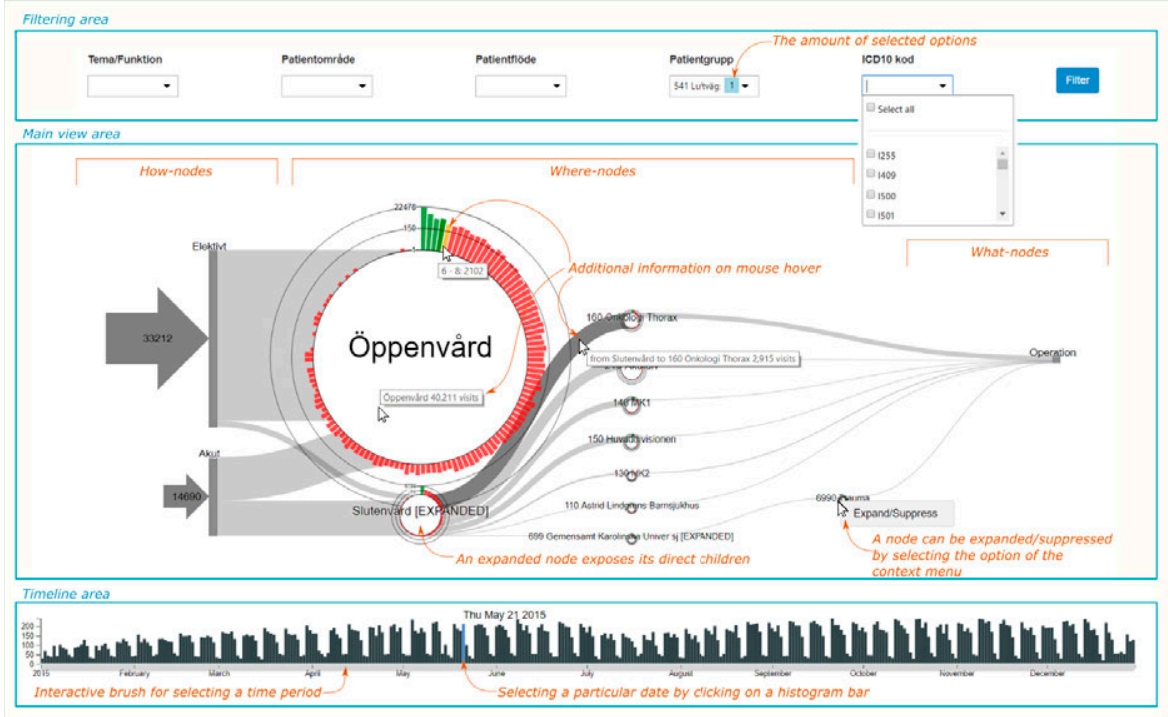

Figure 4.8: The AutoMed web portal: the patient flow overview.

The intelligent alarm management tool is intended to visualize the data, i.e. alarm sequences, generated by mining algorithms. The aim of the tool is to facilitate reviewing and analyzing the detected sequences, i.e. knowledge phase of the VA process as according to Keim's model [134]. For this purpose, a set of visualizations were developed to visualize various types of alarm sequences, i.e. sequential alarms, see Figure 4.7, chattering alarms, etc. These visualizations 


\section{CONDUCTED WORK}

are examples of how initially not user-readable data can be presented in a compact and comprehensive way allowing the user to perform advanced data exploration. The main challenge of this work was to find appropriate visualizations to depict complex sequential data accompanied by multiple time-related attributes. The most notable achievements of the visualizations are a) visual data aggregation of large alarm bursts and b) visualization of time uncertainties. Furthermore, the interface allows annotating the data, e.g. marking alarms that should not be delivered to the operator. Potentially, such annotation can be used for training machine learning algorithms and better configuring industrial alarm systems.

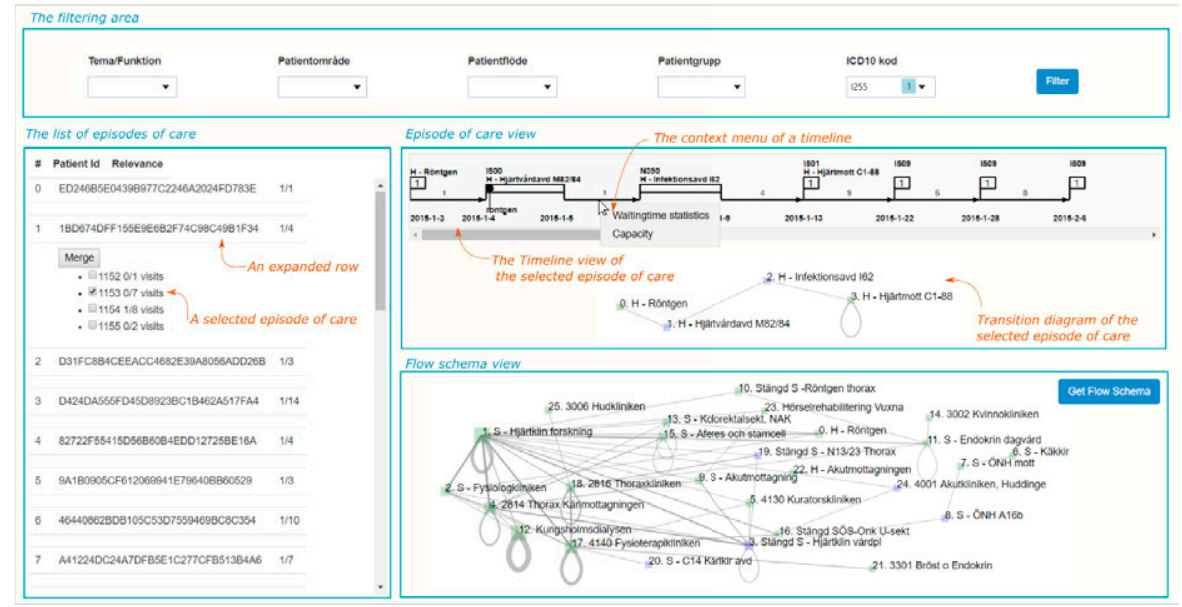

Figure 4.9: The patient cases view and the transition view.

The AutoMed web portal consists of several visualizations allowing interactive medical data exploration, namely the patient flow overview and the patient cases view. It intends to facilitate visualization stage of the VA process as according to Keim's model [134]. The patient flow overview, see Figure 4.8, is a hierarchical data visualization showing the structure of the hospital and the number of acute/scheduled patients that visited each department over a selected period of time. Each department can be expanded or compressed 
through mouse interaction to show/hide the underlying structure of the hospital. The spectrum of waiting times is displayed as histograms around each department. The visualization of patient cases, see Figure 4.9. depicts in detail the disease history of particular patients. The timeline visualization of a disease shows its flow in time, including diagnoses, visited departments, received services and waiting times. Finally, the visualization of patient transitions between departments shows patient transitions in the hospital. The departments and transitions through which many people have passed are visually enlarged. By hovering over the visual elements with the mouse, the user receives precise numerical information. All three views allow applying varying filters so that the user can identify departments and transitions that are related to a specific disease. The overview of patient cases allows advanced interaction, such as merging several cases into one and marking the visits as relevant or irrelevant to a particular decease flow. The interactivity is a step towards annotating the data to be used later on for training machine learning algorithms.

\subsection{Interactive spaces}

Four concepts developed in the course of my PhD contributed to my research on interactive spaces in industrial control rooms, namely 1) the concept of interactive zones [52] developed in the scope of the future operator interaction project, 2) two tangible devices [56] developed in the scope of the tangible interaction project, 3) the fidgeting device [50] developed in the scope of the human-automation interaction course, and 4) two visual aid systems [51] developed in the scope of the SUM project.

In the future operator interaction project, I worked on alternative interaction modalities that could become additional interaction means to the standard interaction through a keyboard and a mouse. My primary goal was to make the operator less tied to the control station 


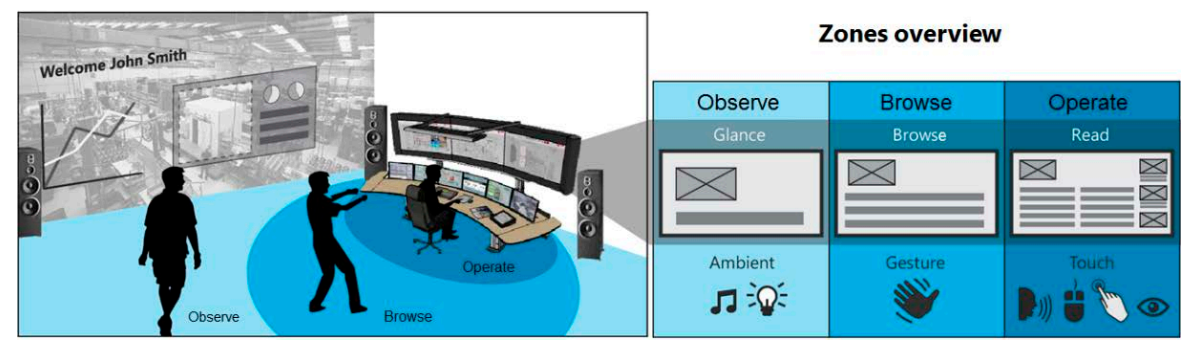

Figure 4.10: The interactive zones concept. The figure illustrates the three interactive zones and the interaction means available to the operator in each of them.

and less dependent on the standard keyboard-mouse-screen interaction. The main outcome of this work is the concept of dividing a control room into a set of interactive zones, see Figure 4.10. The introduced zones roughly correspond to Hall's interpersonal distances [96], i.e. the closest to the station zone corresponds to the human personal space; the second zone, i.e. several meters away from the station, corresponds to the social space in Hall's model; the farthest zone corresponds to Hall's public space. In each zone, a set of interaction possibilities is available to the user based on their proximity to the station. Voice, gaze, palm gestures, keyboards and mice are the options when the user is rather close to the station, i.e. in Zone 1; body gestures are available when in Zone 2. In Zone 3, the user is only allowed to observe the information but not to interact with it. The bottom-line principle is that, in line with the principles of interpersonal communication, the user should approach the station to have more interaction possibilities. Furthermore, the system delivers alarm notifications in a form of audio and light signals to the corresponding zone of the user's presence. Finally, the system adjusts the process graphics according to the location of the user; the farther away from the station the user is, the more enlarged and more generic the graphics becomes. The zones concept promotes untethering the operators from their dedicated workstations allowing them to maintain sufficient SA and be able to interact with the workstation regardless of the distance to it. 
The project on tangible interaction resulted in two tangible concepts, namely the tactile mouse, see Figure 4.11, and the shift report button, see Figure 4.12. The tactile mouse was intended for conveying tactile sensations related to physical attributes of industrial machinery and processes, e.g. temperature, vibrations, etc. My original assumption was that, perhaps, feeling such a reproduction of the original industrial physical effects could help operators to develop a deeper connection with the machinery and processes they are operating. The tactile mouse concept is based on a conventional PC mouse that was extended with several actuators, namely 1 ) a heating element for conveying temperature sensations, 2) a vibromotor recreating the sense of speed or vibrations, 3) a fan producing air flows to simulate liquid or gas flows, and 4) a LED lamp changing its color in correspondence with the status of the hovered object or the current view of the control system, i.e. normal or alarming.

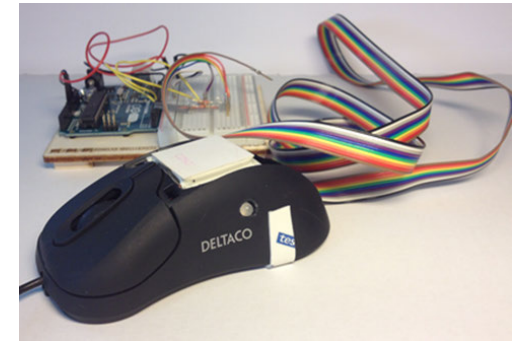

Figure 4.11: The tactile mouse prototype.

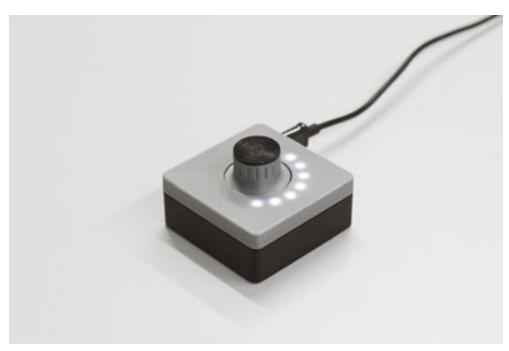

Figure 4.12: The shift report button prototype.

The shift report button was intended to assist operators in the shift reporting process, which at the moment is primarily manual. The developed tool, see Figure 4.12, is a stand-alone device that enables the user to take instant screenshots of all the screens of the operator workstation. The design of the artifact was inspired by emergency pushbuttons that can be encountered in various production environments; whenever an abnormal situation occurs in the production process, a field worker can push such a button to immediately stop or slow down the involved equipment, e.g. a conveyor. By pressing the shift report 

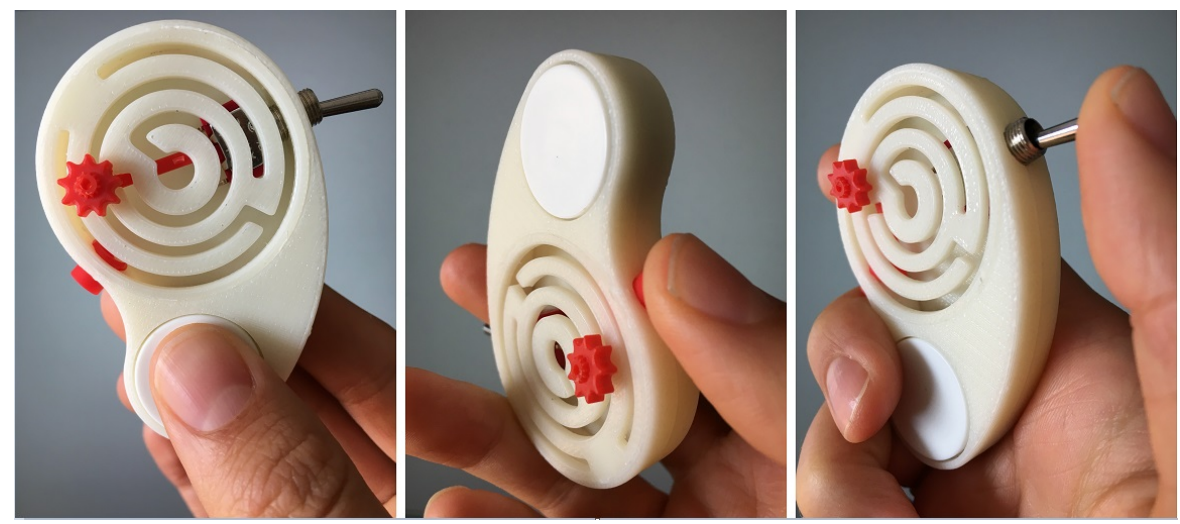

Figure 4.13: The fidgeting device.

button, the operator triggers the system to take screenshots from each screen of the operator's workstation. The screenshots together with their timestamps are saved locally, whenever the user wants to bring them back, they should push and hold the button forcing the screenshots to appear on the same screen where they were taken.

My work in the human-automation interaction course project resulted in a tangible remote control with fidgeting features, which is intended to control an interactive lamp, see Figure 4.13 . Even though the device is not designed exclusively for industrial control rooms, it targets knowledge workers in general. The idea is motivated by the tendency of people to fidget when performing knowledge work. The device allows fidgeting with itself, as well as controlling the light, and "playing" with the light. The device has several control elements: an on/off button to switch the light on and off, a mode switch for switching between the control and fidgeting modes, a labyrinth with a movable pin for changing the color of the light, and a bearing for dimming the light. By fidgeting with the device when it is in the control mode, the user will actually play with the light, e.g. when repeatedly switching the on/off button, the user will make the light blink; when spinning the device, the user will make the light fade or brighten; when navigating through the labyrinth, the user will make the light smoothly 


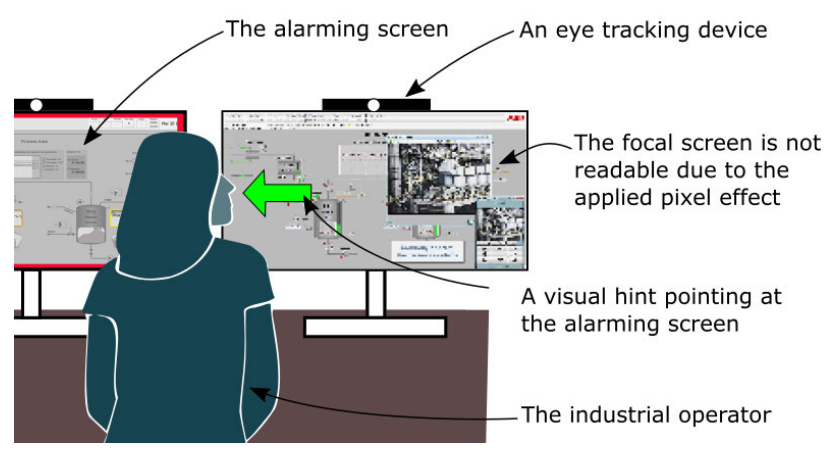

Figure 4.14: Guiding the operator's attention by making the operator's focal screen visually unreadable and showing a visual hint towards more urgent information.

change the color. The device is an example of technology that is designed taking into account the context where it is intended to be used. When working on the physical form of the fidgeting device, I relied on the Norman's idea of perceived affordances [171]. By trial and error, I created the shape that invites the user to perform intended actions. For example, I made the shape visually unbalanced, i.e. with a wide upper part and a narrow bottom part, to show the user that the device can be rotated and encourage the user to do so. Furthermore, I made the shape curvy to motivate the user hold it in certain ways.

Finally, the work on guiding the operator's attention resulted in two concepts and implementations of visual aid systems intended to help the user to attend to the most important information of the moment. Both of them take into account the strengths and limitations of human visual perception. The first system, see Figure 4.14 , is intended to encourage the user to stop their current activity and shift their attention to a more important issue. The system disengages the operator's attention from the focal screen by making the screen unreadable and guides their attention to more urgent information by a visual cue. This approach is intended for rather severe cases when the user must stop whatever they were doing and attend to a more important 


\section{CONDUcted WORK}

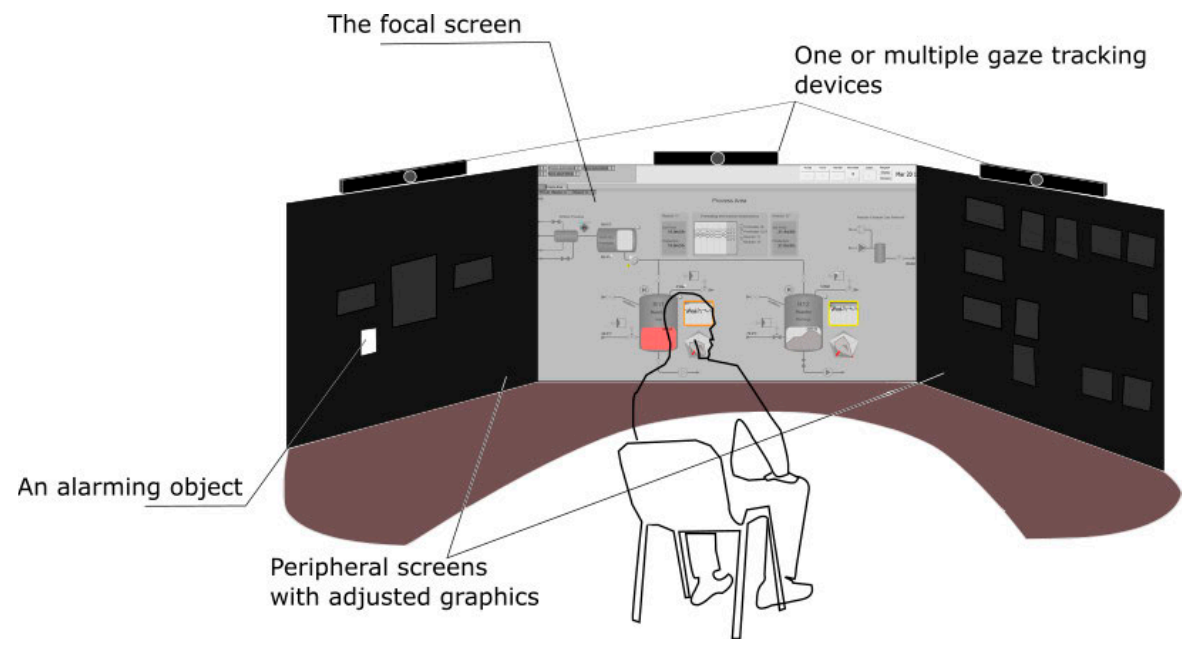

Figure 4.15: Helping the user to attend alarms on peripheral screens by adjusting the process graphics for the peripheral vision of the user.

issue, e.g. an extremely urgent alarm which must be attended to as quickly as possible. The second system, see Figure 4.15, is less intrusive, it is intended to help the operators to notice alarming objects on peripheral screens, however, it does not require them to immediately stop their current activity. The system approach relies on human's fo$\mathrm{cal} /$ peripheral vision specifics. The user evaluation tests proved both approaches effective, however, the users were more positive to the mild approach as they did not like being interrupted in the middle of their ongoing task. 


\section{Chapter 5}

\section{Results}

The prototypes developed in the scope of my PhD are of different types, target various industrial domains and solve diverse problems. Nevertheless, all of them contribute to one or several research questions defined in this PhD, see Table 5.1. Further in this chapter, I dedicate a separate section to each of the research questions where I outline how far I have come in my research.

\subsection{RQ1: How to support the operator's situation awareness under information overload.}

The abundance of screens and wealth of system views, also a constant flow of information and alarm notifications, challenge the operator's ability to maintain sufficient SA. Furthermore, having the control station as the main source of information, operators are tethered to it and lose SA the moment they leave their workplace. In my research, I approached these problems by means of creating a) compact but comprehensive overview screens, b) adaptive systems that adjust data pre- 


\section{RESUlts}

\begin{tabular}{|l||c|c|c|c|}
\hline \multicolumn{1}{|c|}{$\begin{array}{c}\text { Research } \\
\text { question }\end{array}$} & $\begin{array}{c}\text { RQ1: } \\
\text { suppor- } \\
\text { ting SA }\end{array}$ & $\begin{array}{c}\text { RQ2: } \\
\text { facili- } \\
\text { tating } \\
\text { inter- } \\
\text { action }\end{array}$ & $\begin{array}{c}\text { RQ3: } \\
\text { reducing } \\
\text { infor- } \\
\text { mation } \\
\text { load }\end{array}$ & $\begin{array}{c}\text { RQ4: } \\
\text { reintro- } \\
\text { ducing } \\
\text { physi- } \\
\text { cality }\end{array}$ \\
\hline \hline Marine Portal & $\mathrm{x}$ & - & - & - \\
\hline $\begin{array}{l}\text { WARA-PS } \\
\text { control system }\end{array}$ & $\mathrm{x}$ & - & $\mathrm{x}$ & - \\
\hline Overheated Robots & $\mathrm{x}$ & - & $\mathrm{x}$ & - \\
\hline $\begin{array}{l}\text { Intelligent Alarm } \\
\text { Management }\end{array}$ & - & - & $\mathrm{x}$ & - \\
\hline Animated Pipelines & $\mathrm{x}$ & - & $\mathrm{x}$ & $\mathrm{x}$ \\
\hline Medusas & $\mathrm{x}$ & - & $\mathrm{x}$ & $\mathrm{x}$ \\
\hline AutoMed & - & - & $\mathrm{x}$ & - \\
\hline $\begin{array}{l}\text { Interactive Zones } \\
\text { Concept }\end{array}$ & $\mathrm{x}$ & $\mathrm{x}$ & $\mathrm{x}$ & - \\
\hline $\begin{array}{l}\text { Visual Aid } \\
\text { Systems }\end{array}$ & - & $\mathrm{x}$ & $\mathrm{x}$ & $\mathrm{x}$ \\
\hline Tactile Mouse & - & $\mathrm{x}$ & $\mathrm{x}$ & - \\
\hline Shift Report Button & - & $\mathrm{x}$ & $\mathrm{x}$ & - \\
\hline Fidgeting Device & & & & - \\
\hline
\end{tabular}

Table 5.1: The mapping between the developed artifacts and the research questions they address.

sentation and interaction means according to the operator's situation, and c) attention guiding techniques.

In my work, I have developed several overview screens for runtime monitoring, namely the Overheated Robots overview screen [61], the Marine web portal [250], the WARA-PS dashboard [54], the Medusas and the Animated Pipelines interfaces. In my eyes, the power of overview screens as compared to process graphics is that they consolidate all the necessary high-order information on one view relieving the operator from the necessity of switching between different system views to get a holistic understanding of what is going on in the production. Taking into account the limited human perception, the challenge is to deliver the amount of information that the human is 


\subsection{RQ1: How to support the operator's situation awareness under information overload.}

capable to process at once. To address this requirement, in my practice, I followed Shneiderman's Visual Information Seeking Mantra, i.e. provide an overview first, enable zoom and filtering, then provide details on demand [206], as the main underlying principle in the design of my overview screens. On one hand, this approach is especially useful when dealing with large amounts of data as it promotes dividing the information perception process into a series of sequential steps and thus reduces the amount of initial information for the user. However, the approach does not help to answer the questions of what information should be visualized on the screen and in which manner. As the amounts of data grow, designing a meaningful overview while keeping the display complexity low becomes an arduous task. To answer the first question, I was relying on the domain knowledge that I gained from conducting field studies, interviewing operators, as well as studying their existing tools. With respect to the second question, I have practiced custom visualizations, as well as conventional dashboards. My practice shows, that both approaches are valid and cope well with providing essential information in a compact way. From the implementation perspective, dashboards are more straightforward and easy to implement. On the other hand, the tendency of dashboards to divide the content into areas makes them rather limited in depicting very large amounts of information. On the contrary, custom-tailored visualizations bear much more potential in this sense and can present large amounts of data by means of visual aggregation and metaphors. Their drawback is that the implementation is timeconsuming and effortful with respect to finding the right visualization encodings.

Adapting the control station to the particular situation of the operator is another approach to helping the operator maintain good SA in all circumstances. My concept of interactive zones [52] allows the operator to freely move in the control room, as well as leave it, while still getting informed about critical changes in the system via the means of target alarm notifications and adjusted screen views and being able to 


\section{Results}

retrieve more information on demand through more flexible interaction. Changing the look of process graphics depending on the operator's location with respect to the control station allows the user to be able to see the information while being on a distance. Delivering targeted notifications makes the operator more confident in being aware of what is going on in the system. Ability to interact with the station even when on a distance gives the operator the necessary freedom to perform other tasks while staying aware and keeping an eye on the station. The concept is an example of a highly automated system that makes decisions based on the knowledge of the user's context. The implemented high-fidelity prototype of the concept demonstrated its feasibility and practicality in a lab environment. Nevertheless, in the real context, the system would inevitably face a multitude of typical pitfalls of automation, i.e. sensor failures, lack of understanding of the user's context and intentions, disturbances in the environment, etc. To deal with these limitations, the system should empower the user with full control over deactivating the automation at any point in time.

Techniques for guiding the operator's attention to particular information are another powerful means in helping the operator to maintain situation awareness and pay attention to the information most urgent at the moment. Such techniques help overcome the issues of the selective nature of the human's attention. They aim to ensure that the operator actually pays attention to a notification, finds the original problem on a control station screen and starts working on it. In my research, I have outlined two major types of guiding attention, namely guiding the operator's attention to particular information within one screen and guiding the operator's attention to a particular screen. In my research practice, I have implemented both approaches. In the overheated robots visualization, I attract the user's attention to the most important information on the screen, i.e. overheated robots, by visually enlarging alarming fleet items and showing them in more details, also placing them in the very center of the interface creating the fisheye effect; I depict not alarming fleet items in the periphery of the 


\subsection{RQ1: How to support the operator's situation awareness under information overload.}

screen using less arousing visual encodings of a significantly smaller size. This simple but powerful approach to information visualization allows the user to instinctively separate between more and less urgent information and naturally attend to the former. With respect to guiding attention to a particular screen, I have explored two approaches. Both of them are examples of adaptive systems that adjust the control station interfaces to ensure that the user is noticing the most urgent information at the moment. The first approach, more radical, implies 1) making the current focal screen unreadable and 2) showing a visual cue pointing at the screen with more important information. The second, more mild approach is based on the specifics of the human's visual perception; it adapts the interfaces of the peripheral screens to the peripheral vision of the human counting on the natural human's ability to attend to motion and flickers in the periphery. The fact that users preferred the second, mild approach of guiding their attention to the first, more imperative is a sign that people tend to prefer to have control over automation. The second system can be seen as an example of Shneiderman's human-centered automation [205] were the automation creates the circumstances for the user to make less mistakes but leaves the control in the hands of the human.

All in all, my concepts mainly focus on level 1 of Endsley's SA model, i.e. they help the operator obtain the necessary information to maintain sufficient SA. In many respects my solutions comply with some of the interface design criteria for enhancing situational awareness proposed by Endsley [72], namely: criterion 1) allow information organization in correspondence with the operator's personal needs: this criterion matches with my customizable dashboards, criterion 2) allow aggregating information from multiple sources on one view: this criterion matches with my work on overview displays, criterion 3) reduce the need to do manual calculations: this criterion resonates with my work on functional visualizations that eliminate the need to deal with numbers, criterion 4) employ indicators and visual cues to cap- 


\section{RESUlts}

ture the human's attention: this criterion correlates with my work on guiding the operator's attention.

\subsection{RQ2: How to facilitate the operator's interaction with the operator workstation.}

The modern operator workstation setup is restricting the operator in a variety of ways. From my standpoint, the two major limiting factors that make the life of the operator complicated are 1) the control station lets the operator interact with it only when they are in its close proximity, 2) the existing legacy interaction means, i.e. keyboards and mice, are in many ways limiting and prevent the user from freely and spontaneously interacting with the control station.

I see one way to address the restrictive nature of the control station in expanding the interactive area around the station by means of advanced sensors. In my work, I have implemented this idea in the concept of interactive zones [52]. My approach adds more flexibility to the operator who now can interact with the control station using a variety of natural interaction means also when being on a distance from the station. Relying on the basics of interpersonal communication [96] allowed making the underlying principles of the system straightforward to the user as they go inline with their habits of natural interaction. My experiments with the sensor-based technologies revealed one significant problem, namely that they are not fully reliable to be applied in real industrial settings. The problem is more complicated than error-prone sensors. For example, it is not clear how to distinguish between a person making a control gesture and a person actively gesturing when talking to a colleague. Potentially, advanced machine learning algorithms could be of help, however, even they are prone to mistakes. After all, it is only the human who can be com- 


\subsection{RQ2: How to facilitate the operator's interaction with the operator workstation.}

pletely sure when s/he is making an intentional gesture to control the station.

In addition to sensor-based technologies, I see tangibles as another promising alternative to keyboard and mice interaction. I believe that tangibles could play the role of shortcuts for interacting with overly complicated control stations and their interfaces. Such devices could help to achieve outcomes that would normally require a long sequence of actions using conventional interfaces. For example, my work on the shift report button [56] has demonstrated the potential of using a physical interface for taking screenshots for shift reporting. Normally, such an action would require the user to 1) find the right keyboard, 2) press the necessary combination of buttons to capture a screenshot, 3) save the screenshot to some local folder on this or another PC. The shift report button allows achieving the same result in one button push. Another valuable property of tangibles is their ability to engage the user in physical activity; it is rather natural for humans to interact with the environment with their hands. My work on the fidgeting device [50] demonstrates how a system control can be designed taking into account the human's habits to fidget while doing knowledge work.

Regardless of the seeming benefits, there are also several drawbacks that I could outline with respect to tangible interfaces. First of all, as they require physical contact similarly to a keyboard or a mouse, they will inevitably get dirty in an industrial environment and repel the users. There is another challenge in the temptation to create different physical interfaces for different shortcuts. The risk is to end up with a collection of diverse artifacts that will cause confusion and misunderstanding leading to being abandoned in the corner of the desk. A potential solution to this problem could be to locate multiple physical controls on a centralized physical control panel.

All in all, in my attempt to expand interaction means in the context of industrial control rooms, I tried to go beyond the conven- 


\section{Results}

tional confines of the keyboard-computer-mouse-desktop interaction and looked for interaction means that would incorporate themselves into the physical and social world of the industrial context. In my research practice, I tried to follow the principles of embodied interaction [62]. First of all, I aimed to leverage natural human abilities of manual manipulation and gaze, gesture and voice interaction. Furthermore, I tried to incorporate knowledge about the industrial context into the interaction. For example, in the case of the shift report button, pushing the button will reveal to others in the control room that something extraordinary has just happened in the control system. With regard to the interactive zones concept, when experimenting with various modalities of interaction means, I tried to avoid blindly adding them in the context of the industrial control room merely because they are trendier than the conventional keyboard and mouse. In my eyes, thoughtlessly adding alternative interaction means will result in more complex control rather than efficient interaction with the control station. Instead, I tried to find a reason behind each newly introduced modality and how it will fit into the context of industrial control rooms. For example, voice, gaze, and palm gestures are of use when the operator is wearing safety gear or has dirty hands and therefore they cannot use a mouse or a keyboard. Perhaps, the fidgeting device is the most obvious example of thoughtful incorporation of new technology in the target context. The shape, as well as the functionality of the device, target the context of knowledge work and orient towards a typical behavior of the user in the given context. 
5.3. RQ3: How to reduce the information overload of the operator.

\subsection{RQ3: How to reduce the information overload of the operator.}

Industrial control stations are overloaded with screens showing interfaces full of numerical and textual information. Such a setup inevitably overloads the user with information. The operator gets extra cognitive load due to demanding interaction with the interfaces, i.e. navigating complex hierarchical menus, remembering how to bring one or another view on a screen, looking for a keyboard that allows controlling a particular screen. In my research, I investigated several methods to reduce the cognitive and visual loads in industrial control rooms, namely a) showing only relevant information and hiding unrelated information, b) expressing some information using tactile sensations rather than visualizations, and c) introducing more natural physical interaction with the control station.

The main approach I practiced to reduce the amount of visualized data presented to the operator is by showing only important information and hiding data that is irrelevant at the moment. One way to achieve this goal is to design overview screens that aggregate and present only important high-order information providing the remaining secondary data on demand, as according to Shneiderman's Visual Information Seeking Mantra [206]. In my eyes, an effective overview screen could potentially replace the need of having multiple displays showing too detailed information. My practice shows that this approach is applicable for interfaces intended for both real-time status monitoring and Visual Analytics purposes. Importantly, as the ultimate goal is to reduce the cognitive and visual loads of the interface, the approach always implies a number of trade-offs, i.e. what to show directly and what to show on demand. To come up with the visualization approaches, I experimented a lot with combining different data layouts and coming up with ideas of visual markers that would aggregate several dimen- 


\section{RESUlts}

sions of data. Furthermore, I relied on the specifics of human perception. For example, I often visually grouped the data which is in line with people's natural habit to create categories as a way to make sense of the world around them, especially when they feel overwhelmed with information [236]. Another way of hiding irrelevant information is to make it imperceptible. I implemented this approach in one of my visual aid systems where I adjusted the view on the peripheral screens to the peripheral vision of the human [51]. In the concept, I turned the interfaces of the peripheral screens into grayscale, simplified their process graphics to basic rectangular shapes, and made them nearly indistinguishable from the background unless they are alarming. Such design allows the user to focus on their current task not being disturbed by other visual information unless it is urgent.

Another method of reducing the amount of visual data is presenting runtime data in some alternative form rather than in textual and numeric as it is now. For this purpose, I experimented with two approaches, namely a) conveying the runtime data as tactile sensations and $b$ ) showing them as functional visualizations. The first approach was implemented in the tactile mouse prototype [56] where a set of embedded actuators were intended to replicate some of the industrial sensations in a smaller and milder scale. The idea looked very promising in the beginning, but later raised more questions than answers. First of all, production parameters in the industry have extreme values, e.g. high voltage, very high or low temperatures, high-frequency vibrations, etc., that one cannot directly convey through tactile interfaces. Obviously, a mapping is needed to map such extreme values to the ones that could be tolerated by a human body. Such mapping, however, implies that a spectrum of the original values will be mapped to one value in the tactile interface leading to lower precision. Furthermore, the visual perception of numeric values is faster and more precise than tactile. For example, to see and comprehend a visual value takes seconds, while to 1 ) feel a tactile sensation, 2) estimate its numerical value, 3) mentally convert it to the process value, 


\subsection{RQ3: How to reduce the information overload of the operator.}

and finally 4) make a conclusion whether the value is below or above a certain threshold would take much longer time. Also, the user is likely to estimate only an approximation of the original value as it is impossible for a layperson to differentiate between say 30 and 30.5 degrees through tactile sensations. In the industry, where speed and precision of making decisions are essential, tactile interfaces may be at disadvantage. The case is getting complicated further with personal perception specifics and body shapes. For example, some people are more sensitive to some stimuli than others. Moreover, human perception is prone to various biases. Imagine, for example, coming from a cold environment, your hands are freezing, touching any warm object would feel much warmer than it is in reality. Finally, there are purely technical issues. For example, it is technically challenging to make a tangible device get instantly very warm or very cold while remaining safe for usage. Such a device would need access to a relatively high voltage which would make it potentially dangerous. Based on these considerations, I have decided to abandon working with tactile interfaces for the purpose of replacing visual KPIs. A more fruitful approach in my eyes is to employ functional visualizations that tend to depict numerical values by purely visual means. I used this method in my work on overview screens for the district heating domain [55]. Effective multidimensional visual encoding can allow having one functional visualization that depicts multiple status variables simultaneously. One disadvantage of this approach is that it usually requires a long ideation process.

To reduce the cognitive load of interacting with the control station and its interfaces, I experimented with natural interaction means such as gaze, voice, and gestures, as well as physical interfaces. In the interactive zones concept [52], gaze, voice, and gestures were alternatives to the conventional mouse operations such as clicking through a menu or selecting an object of interest. In addition to cognitive ease, the operator does not need any physical device to perform such actions which facilitates the interaction. Physical interfaces, such as the Shift Report 


\section{RESUlts}

Button [56] and the Fidgeting device [50] rely on the human's natural ability to interact with their hands. As was mentioned earlier, they are embodiments of shortcuts in achieving desired actions in the system meaning that the desired action can be achieved in a less amount of time and with relative ease.

\subsection{RQ4: How to bridge the gap between physical industrial processes and their digital representation.}

My research on the topic of bridging the gap between physical industrial processes and their digital representation started with arguably the most straightforward way of conveying physicality, namely through tactile feedback. I implemented my ideas in the tactile mouse prototype [56]. Compared to the previously discussed application case of replacing visual KPIs with tactile sensations, the goal here was to give the user only the feeling of a certain physical phenomenon but not its particular numeric value. The development of the tactile mouse concept has proven the technical feasibility of such an approach. However, the user evaluation of the tactile mouse revealed some problems that make this approach, in my eyes, inexpedient. The first problem is the need for appropriate configuration of the tactile sensations. From the user evaluation of the tactile mouse, it became clear that even though the operators were not against feeling some tactile sensations, their opinions about the intensity and timing of such feedback, i.e. when and for how long it should be delivered, significantly differed. On one hand, the interfaces should not be harmful or disturbing to the operators, but on the other hand, everyone has their own criteria of what is disturbing. Potentially, there are ways to 


\subsection{RQ4: How to bridge the gap between physical industrial processes and their digital representation.}

address this issue, e.g. by providing personalization possibilities, but I doubt that operators will be willing to configure a device every time they take over the shift since the devices are shared. Furthermore, the situation is complicated by the necessity to have a physical contact with a device or wearable which is not always desired especially in the context of industrial settings where everything tends to get untidy and the interfaces are shared. The negative feedback received from the user evaluation made me doubt whether the operator sitting in a safe and calm office environment actually needs sensory feedback from the industrial process they are monitoring. During the ongoing transition from the analog to digital control rooms, there was some evidence in the literature that industrial operators were regretting the transition and missing the physical contact with the production [251]. However, in later works, there was no sign of operators complaining about the issue. Perhaps, that original regret was a part of the transition period which imposed extra pressure on the workers of that time forcing them to adapt to the new environment and new ways of working which required an effort and made them regret the ongoing changes [1].

As an alternative to physical sensations, I have investigated another approach of expressing the physicality of industrial processes, namely using purely visual means. The idea is to include visualization of physical properties into the process graphics or, in other words, make the visualization of the industrial process trigger some physical associations or even sensations. The approach is especially valid for overview screens which are not intended to be elaborated in detail, thus have space for artistic digression. I implemented some of these ideas when working on the interfaces for the district heating domain [55]. There I have extensively worked with expressing the physical properties of water relying a lot on metaphors, colors, and animation. I believe that this approach is much more powerful compared to tactile interfaces as it opens many more possibilities for expression. Further- 


\section{Results}

more, the resulting interfaces are less intrusive compared to the tactile sensations. 


\section{Chapter 6}

\section{Discussion}

Now, when all the intended projects of my PhD research are finished, it is time for reflection on the conducted work. The purpose of this chapter is twofold. In the first section, I want to critically assess the methods I have applied in my research. Furthermore, I intend to analyze how the original constraints of my PhD setup, namely being affiliated with a business company, influenced my research workflows and results. In the second section of this chapter, I would like to reflect on the developed artifacts and give a sketch of one potential setup of future control rooms where my artifacts would be sensible parts.

\subsection{Methodology}

\section{UCD practices}

In my research, I was practicing $\mathrm{RtD}$ as the main approach, in particular the field genre of RtD meaning that my projects originated from real users and their problems. However, the particular research flow in each project, as well as the preferable selection of research methods, were defined by a variation of the UCD process practiced in the UX group at $\mathrm{ABB} C \mathrm{CRC}$. With respect to my research practices, the UCD 
process as such was not particularly restrictive and allowed a certain freedom of action. For example, the order of the process stages was rather recommended, some stages could be omitted, also particular methods on each stage were not strictly defined. Most importantly, the process did not specify how the knowledge retrieved on each stage should be interpreted and used on the next stage. Having these freedoms at hand, I had possibilities to control my research process in correspondence with the needs of my research. In this section, I would like to reflect on the research methods that I have used on the four major stages of the UCD process, i.e. 1) field data gathering and analysis, 2) ideation, 3) prototyping, and 4) evaluation.

With respect to field studies, I believe, they played a significant role in my research. The data collected during visits to the industry was both informative and inspiring to me. On the other hand, as my ultimate goal of getting familiar with the industrial context was to see a general picture rather than focus on particular workers' problems, at a certain point in time, I was satisfied with the knowledge I had and was not very excited to visit yet another control room. Based on my experience, I concluded that, if to take away attention from the physical setup, all industrial control rooms are quite alike in terms of the workflows and activities happening there. Furthermore, reaching sufficient knowledge, I started feeling comfortable with relying on materials collected by other researchers because I could assess it critically. This was also positive from the perspective of avoiding unnecessary traveling to the field.

With respect to the ideation process, access to industrial personnel had both encouraging and "sobering" effects on my research. On one hand, the materials collected during field studies, as well as live impressions from the field, were often a source of inspiration opening up the potential for innovation. On the other hand, knowing the real context, as well as being familiar with industrial workflows, I often felt a need to stay realistic and "down to earth" in my ideation process. Furthermore, the exposure to the real user often strengthened the temptation 
to focus solely on their direct needs rather than thinking conceptually outside of a particular user's problems. There is no doubt that focusing on problems of existing tools and interfaces, one can develop needed improvements that can make the lives of industrial workers easier and their workflows more productive. Examples of such innovation in my research are the dashboards developed for different industrial domains, e.g. the marine web portal [250] and the dashboard of the WARA-PS demonstrator. However, in my eyes, such an approach leads to rather "lazy" innovation when the researcher is doing simply what they were directly or indirectly told or pointed at by the users, i.e. they take the opportunities laying on the surface instead of making an effort to search for hidden implications. Importantly, such research does not necessarily mean less effort, but it will inevitably lead to merely pragmatic and straightforward improvements of existing tools rather than would open up new design horizons.

I believe that, for more broad ideation, seeing the environment and talking to workers is barely enough. More input is needed to be creative. In my opinion, innovation is born at the intersection of multiple knowledge domains often not directly related to each other. In my case, such additional knowledge was my expertise in state-of-the-art technology, also my skills in art and design, as well as my familiarity with cognitive sciences. The technical skills gave me the opportunity to spot innovation possibilities in the outdated environments of control rooms, as well as assess what would be technically possible to implement. In general, the industrial domain is a "fertile ground" for research aiming at introducing new technology. The industry is rather slow in adopting innovation, therefore, a technology that is commonplace on the consumer market might still be perceived as novel in the industrial domain. The challenge is to find a reasonable application case for the technology. An example of such technology-based innovation in my PhD research is the zones concept developed in the Future Operator Interaction project [52] where a selection of state-of-the-art interaction technologies was introduced in an industrial control room 
environment to extend the existing limited interaction. However, I see this type of innovation, i.e. enhancing old workflows with new technology, as self-evident and therefore limited. The possibilities to innovate will eventually end when the potential of new technology replacing old tools and methods is fully exhausted. I believe that, for reaching more radical innovation, in addition to knowing the context and state-of-the-art of the technology, one needs to involve other knowledge areas that are not directly related to the industrial domain. In my case, those were art and cognitive science domains.

My knowledge of art techniques allowed me to apply artistic methods in the design of artifacts. One example of a solution with artistic touch is the overheated robots interface where I use principles of artistic composition and perspective to lay out a large fleet of machinery. Furthermore, my skills in art allowed me to involve metaphors originating outside of the industrial domain. An example of such work is the Medusas interface [55] where I used my expertise in semiotics, i.e. science about signs and symbols, to design informative icons for the district heating domain.

My interest in cognitive science, especially in bottlenecks of human perception and various biases, allowed me to use some of that knowledge in designing user-oriented interfaces that acknowledge human weaknesses and tend to mitigate them. In the industry, for decades, the prevalent way of thinking was that interfaces and technologies should be functional rather than pleasing the user. The human was seen as a residual must, especially taking into account the growing strive and enriched possibilities for increasing levels of automation of the last decades. As a result, industrial user experience is a significantly understudied domain opening a wide range of possibilities for research. Perhaps, the most evident example of applying cognitive science knowledge in my research is my work on guiding the operator's attention where I converted some specifics of the human's attention into features of a control system interface. 
With respect to research and the quality of generated knowledge, I believe that combining knowledge from multiple domains is a very fruitful approach, but also time-consuming, effortful, and risky. I see the main challenge in integrating seemingly unrelated knowledge into a common concept idea. In this case, the researcher is similar to an alchemist who is mixing several chemicals in one volume having different proportions resulting in different substances. The result might not always be satisfactory or of use and the search for the right combination of "ingredients" can be exhausting, but in the case of success, the result has a chance to become truly novel, unforeseeable, and generative for others. In my research, I could not always reach this level of innovation. The reasons for that were lack of time, lack of inspiration, but most importantly the applied, i.e. business-oriented, nature of my projects where innovation interesting from the research perspective was not always welcomed.

In regard to prototyping, in my research, I have practiced both lowand high-fidelity prototyping. Based on my experience, I believe that both approaches have their pros and cons. Low-fidelity prototyping is rather cheap and fast, but also unfinished in detail thus ambiguous. On one hand, the latter is beneficial because the researcher has a chance to focus the test user's attention on the holistic concept idea of the artifact rather than on its look and feel. On the other hand, the user will imagine missing parts, characteristics, and functionality of the artifact which will inevitably add uncertainty to the evaluation. As a result, it might be difficult to ensure that the researcher and the test user share exactly the same mental model of the concept. The user's feedback will relate to the conceptual model they have in their mind which might be significantly different from the original concept intended by the researcher. In my practice, I had some misunderstandings of this sort with the stakeholders of the City Control room project. On an early project stage, when discussions were organized around paper sketches of future concepts, both me and the stakeholders were satisfied with the results and fully convinced that we are on the same page 
and moving in the right direction. However, in the later prototyping phases, many conflicts of interest popped up which obviously originated from unrevealed misunderstandings in those early low-fidelity prototypes.

On the contrary, high-fidelity prototypes do not leave much freedom to the test user's imagination. The results of a user evaluation will elicit the user's attitudes to the particular artifact at hand. A significant drawback is that the finalized look and feel of the artifact may distract the user from the original concept idea. In my practice, I had a similar experience with the tactile mouse concept. When arranging a user evaluation, my goal was to test whether the overall idea of having haptic feedback in a mouse device would be appreciated by industrial operators. The look-and-feel of the test prototype was rough, but the functionality was working as intended. Despite my intention to get feedback regarding the overall acceptance and appeal of the idea, the users kept commenting on the design of this particular prototype at hand, e.g. the locations and intensity of the actuators. Their feedback was mostly negative which resulted in the overall skepticism towards the concept.

In relation to the evaluation of my artifacts, I mostly practiced qualitative evaluation with real users and in real industrial settings. However, my evaluation methods were rather preliminary. At most, they reveal whether the solutions were appealing to the particular industrial workers, but they do not necessarily show if the prototypes would be actually accepted and used in the real workflows. Getting a realistic picture would require the prototypes to be integrated into the environment of the users and reside there for a while. Moreover, a proper evaluation would require a quantitative assessment. In general, I see that quantitative evaluation was often the missing element of my concepts to be successful. For example, I could often feel the interest of stakeholders in my ideas, but they were hesitating to proceed with them further because they were not fully convinced of their practicality. I would often perceive it as unwillingness to accept my 
vision, but now I realize that reinforcing the concepts with numerical proof of improved operator's performance could have made the stakeholders more open to my innovations. In my defense, I should say that a) running a quantitative evaluation with industrial operators was hardly ever an option due to the reasons mentioned in the Methodology chapter, and b) evaluating most of my concepts, such as visualization, with lay users would always raise a question whether the results can be generalized with respect to the real industrial workers.

In conclusion, I want to add that, even though practicing the UCD paradigm was not my personal choice, I believe that it was a reasonable technique to practice with respect to my research goals that per se were very user-oriented. UCD was always "reminding" me about the user and giving a reason to come back to the user for more knowledge, inspiration or for an opinion about an idea. Importantly, "coming back to the user" did not necessarily mean to physically meet the users, but to act with the user's interest in mind, e.g. when designing an interface, take into consideration the principles of human cognition or introduce elements of aesthetics. It might look that the produced artifacts turned out to be very grounded and practical due to excessive user orientation, but I believe that it was not the influence of UCD as such, but more of the affiliation with the business company which I will discuss in the next section. In any case, it is satisfactory to know that the results of my work can be directly useful to a particular group of people.

\section{Collaborative projects}

Due to the setup of my $\mathrm{PhD}$, my research projects were essentially collaborative between academia and a business company, i.e. ABB. Even though in all these projects I was practicing the same UCD process, the quality of the produced results varied. Some of the projects resulted in novel outcomes and knowledge contributions, whereas in 
other projects I ended up having very practical and rather conventional outcomes. Analyzing the situation post factum, I am inclined to think that the outcomes depended on the "power distribution" between the project stakeholders, i.e. the business company and the researcher; the party that steers the research, shapes the results in their favor and gets the most benefit. Based on my experience, I can outline three possible setups of such "power distribution" that I have encountered in my practice. The first setup assumes that the researcher approaches the business company and initiates a research project taking all major decisions regarding the research process activities. The second setup implies that the business company approaches the researcher and invites him/her to participate in a project, but the business company manages the research process. The last option is when both parties are equally represented in a project aiming to co-create new things together and both are equally in charge of the research process. To better illustrate my observation and to be able to refer to the identified setups, I introduce the notion of co-production design space, see Figure 6.1. In my eyes, the co-production space is a continuum with two possible extremes, namely "business territory" and "research territory", see Figure 6.1. The middle point of the continuum represents a setup when the business company and academia have equal authority in the project. Based on my experience, I am inclined to think that my role as a researcher, the tools that I was using, and the outcomes of my work varied drastically depending on which part of the co-production space the project belonged to. Further in this chapter, I will briefly describe how each particular setup influenced my research routines.

In the first setup, i.e. when the business company was the initiator of the project, the business stakeholders typically used to have certain expectations of the desired results in their minds. Even when they had no concrete vision, by default they would expect something realistic and practical that could become a part of the company's product portfolio. This precondition was significantly restricting my freedom 


\subsection{Methodology}

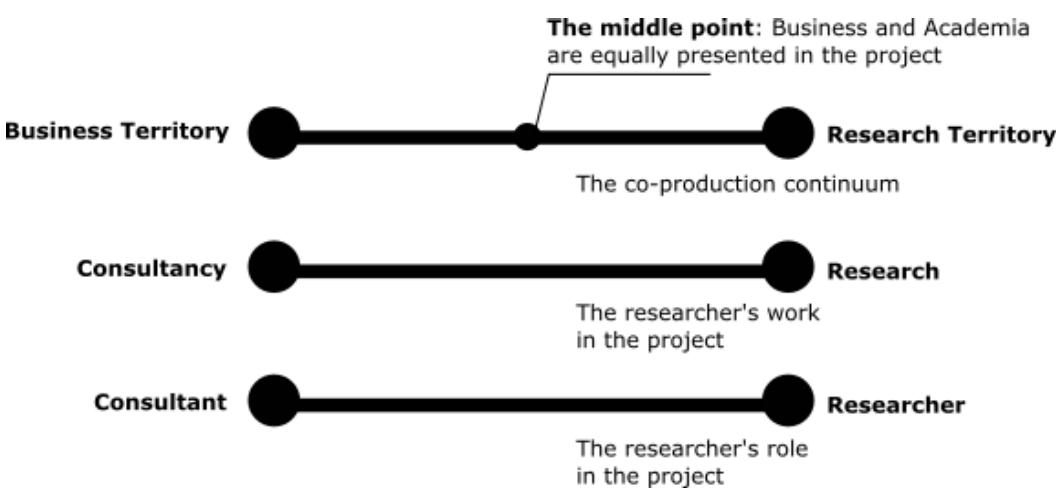

Figure 6.1: The co-production design space.

as a researcher in the project. Field studies were often of importance but more from the perspective of revealing customers' wishes. Emphasis was put on reviewing existing interfaces and ideating around the ways how they could be elaborated or improved. For conveying ideas, I was using sketches and mockups that were rather detailed and straightforward, for example, realistic Photoshop screenshots. For the purpose of prototyping, I was normally using the tools that stakeholders are familiar with; later, that would simplify adoption of the prototype in the production. In general, such project setups were quite limiting in terms of doing actual research and making significant knowledge contributions. I was often witnessing how the research process would turn into consultancy. In the best case, after all the invested time and effort, the work might result in a case study paper published in a conference's industrial track. A more ordinary scenario was that the delivered work could not be disclosed at all due to the secrecy policies of the business company. One example of such work is the marine web portal [250].

In the second setup, the researcher is steering the design process based on their own agenda and research plan. In such projects, I had access to all the privileges of working in collaboration with industry, e.g. I had possibilities to conduct field studies and communicate with users 
and customers, but at the same time, I was less restricted in my decisions. I could choose any methods and any tools for my research practice. In general, I was still orienting myself towards the user needs, but my goals were less pragmatic. I tended to abstract from particular tools or problems at hand, instead, I was looking for novel ideas that would not necessarily be backed by the customer interest or market profits. In such setups, I was much more open to experiments with knowledge from different domains. Examples of such projects are, for instance, my work on the shift report button and the tactile mouse [56], as well as the fidgeting device [50].

The third setup incorporates collaborative projects that are formed through co-determination, where both parties have an equal right to steer the design process and are equally demanding about pursuing their interests. The particular goals of the project, as well as the research process flow, have to be defined collaboratively. Obviously, the parties, who often have opposite interests, need to compromise in order to achieve a consensus. In such projects, my role as a researcher was to generate and propose innovative ideas that would be either accepted or rejected by the industry representatives. The projects usually started by extensive field studies. The ideation process was not restricted, but it was a challenge to justify ideas and get the green light to proceed. Typically, I used the materials collected in the scope of the field studies to motivate my concepts which would make them look grounded in the real user needs. When communicating ideas, it was a challenge to make stakeholders understand them. As my experience shows, the methods for sharing ideas that work in the design community do not necessarily work for industry representatives. For example, sketching turned out to be quite a vague method for communicating ideas to industry representatives, mostly due to its ambiguity, lack of details, and precision. Communicating ideas through sketching would often result in misunderstandings or faulty beliefs. As a result, when participating in such projects, I tended to express my ideas by sketching in code. In general, this project setup provides much 
more freedom compared to the industry initiated projects. The best practice, however, would be to keep ideas implementable and close to reality. One example of such a project is my work on alternative data visualization for the district heating domain [55].

Based on my experience, participation in a collaborative project can be quite tempting for a researcher due to a variety of reasons, for instance, establishing business connections, getting to work on realworld problems, getting access to the field, etc. However, the expectations of the researcher before the project start can be quite different from what the reality might bring. The project that looks very attractive in the beginning can turn into a sidetrack activity draining time and effort and slowing down the researcher in their actual research work. This recurring situation of having high expectations but ending up in uncertainty and frustration motivated me to put my experience on paper. The main lesson learned is that in order to estimate the potential of a collaboration project it is important to judge where in the collaborative space continuum the project will likely take place. Defining which party will be steering the design process, can help to understand the nature of the researcher's work to be conducted and possible outcomes including potential scientific contributions.

\subsection{Future control rooms}

When working on my $\mathrm{PhD}$, I did not have an explicit goal to create a vision of an industrial control room of the future. Also, the research projects conducted in the scope of my PhD were often related to different aspects of industrial operators' work in various industrial domains. Such diversity prevented me from working towards a holistic picture of a future control room. Also, the collection of resulted artifacts is very diverse in their look-and-feel, as well as in their purpose. Nevertheless, when analyzing the conducted work, I see certain conceptual similarities across the developed solutions. On the whole, all 
of them are explorations of similar concerns. In this section, I would like to highlight important features that run through the set of my artifacts and outline resemblances. In line with the best practices of RtD, I would like to assemble a body of my artifacts into an annotated portfolio. However, instead of merely listing similarities and differences, I prefer to abstract from the particular examples and speculate about one possible future of industrial control rooms, i.e. in 15-20 years from now, where these and similar artifacts could be widely adopted. To make a reasonable forecast, I intend to rely on my knowledge about the trends of the ongoing digitalization, the pace of industrial innovation, and the changing nature of industrial workflows.

In my eyes, when trying to predict the future of industrial control rooms, the main factor to consider is the low speed of new technology penetration into the industry. The reason for such conservatism is multifold, namely a) various safety standards that slow down the acceptance of new technology, b) high expenses of required investments, and c) necessity to re-educate and re-train work personnel. As a consequence, I believe that it is unlikely that in the close future, i.e. 15-20 years, industrial control rooms will significantly change their setup and typical workflows.

With respect to the setup of industrial control rooms, I expect that at least for another decade or two control rooms will look similar to how they look today. Operators will continue working in office-like environments on a distance from the actual production. They will have their dedicated workplace, i.e. an industrial control station, which, as today, will consist of a desk and a set of monitors of different sizes and purposes, keyboards, and mice. Alarm systems will remain the main channel to deliver notifications. Process graphics will continue being the main visualization approach to schematically depicting production processes and equipment. However, the continuous digitalization and exponential growth of the amounts of data being delivered to control rooms will challenge the limits of the current interfaces. Extensive adoption of sensors and actuators in industrial processes will 
lead to more complex system views which in turn will result in more sophisticated industrial control stations and their interfaces. Not only will it become hard for the operator to maintain sufficient situation awareness, but it will become challenging merely to interact with the control station as it turns too intricate. Taking into account the human's inability to work with large amounts of data and do multitasking, there will be a need to extend the existing data presentation and interaction approaches to tolerate new conditions. Operators will be seeking helping means capable of assisting them in interacting with the control station and maintaining good situation awareness. One way to address these challenges without significantly changing the overall setup is by introducing add-ons, i.e. extensions on the top of existing control systems. The goal of the add-ons will be to mitigate inefficiencies and emphasize the strengths of the human operator. This is where I see the technologies I have developed in the scope of this $\mathrm{PhD}$ will come in handy.

At this moment, I can outline two major groups of such add-ons depending on their purpose. The extensions belonging to the first group will be intended to aggregate data before delivering it to the users to avoid potential information overload. The reduction will touch upon visual data presentation, as well as notification delivery. With respect to information visualization, existing process graphics will be extended with overview displays containing less information but providing a comprehensive summary of the current situation in the production. Conventional dashboards are the first step towards such an aggregation. Examples of such dashboards in my work are the marine portal [250] and the dashboard for the WARA-PS demonstrator [54]. However, at some point, the capacity of dashboards will become insufficient to cope with constantly growing amounts of data. Instead, overview displays will rely more on holistic visualizations with visual metaphors and functional visual encodings rather than on numbers or texts. Examples of such displays in my work are the overview screens for the district heating domain [55]. With respect to notification dis- 
tribution, alarm systems will be extended with tools allowing to suppress redundant notifications and ensure that only essential messages are delivered. My work on the intelligent alarm management interface [53] is an example of such an extension.

The second group contains extensions that will enhance the operator's interaction with the control station. In addition to conventional keyboards and mice, control stations will have add-ons to open up possibilities for natural interaction, such as voice, gaze, and gestures, similar to the solution presented in my work on interactive zones [52]. Targeted notifications, as presented in [52], is another area of possible extensions, which assumes that the control room will be aware where the operator is at each particular moment and will deliver notifications to the destination in a suitable manner. Thanks to these extensions, the user will be less tethered to their workplace and will be able to choose suitable interaction means depending on the situation, the proximity to the station, and the urgency of the issue. Another family of extensions will incorporate shortcuts in interacting with the control station. Such shortcuts will let the user achieve certain desired outcomes in a faster manner bypassing rigid technology-restrained interaction and long procedures of sequential steps. The shift report button [56] is an example of such a shortcut, namely the user can take screenshots of the control station interfaces by pressing a single button. Voice commands and gestures, as presented in [52], are another example of such shortcuts when the user can rapidly navigate in the hierarchy of system views instead of clicking through nested menus. These extensions will bring the user close to the future where they will be able to interact with the control station in a manner similar to how they interact with people in their everyday life, i.e. spontaneously, freely, and by means of a rich spectrum of interaction modalities.

Importantly, some of these extensions can be introduced independently, but some can be effective only in collaboration with others or under certain constraints. For example, the visual aid systems intended for guiding the operator's attention not only will be inefficient, 
but can even be disturbing without measures for reducing the number of notifications delivered to operators. Also, it is unrealistic to allow the user to interact naturally, i.e. by means of gaze, voice, and gestures, with an overly complicated control station comprising a myriad of sensors, monitors, and interfaces without inevitably increasing unpredictability. To overcome these issues, perhaps, the extensions will start appearing in the control rooms gradually and on a small scale, e.g. they will be available only in a certain area of the control room or will be applicable only to a subset of screens/interfaces of a control station.

With respect to automation, I believe that due to a variety of reasons, many of today's operator workflows will remain manual. Obviously, routine and monotonous tasks will keep getting automated, but the leading role of the user as the decision-maker will not be suppressed by automation. The reasons are related to several facts, namely a) automation is prone to errors and b) it has only partial knowledge of the context and the user's intentions, also due to c) the human-out-of-theloop problem and d) automation bias. Another significant reason is the well-known fact that people are better at sense-making than machines. This superiority was pointed out already in the first versions of MABA-MABA lists [77] and, in my eyes, it will remain for decades. The extension described in this thesis are automated solutions, e.g. the shift report button [56] automates the process of taking screenshots from a multitude of screens, the visual aid systems automatically adjust the screens according to the situation with alarms [51]. However, they are examples of rather low-level automation that tend to create a productive environment for making decisions, but the decisions per se remain in the hands of operators. The overall goal of the aforementioned extensions is to empower the user, capitalize on their strengths, and diminish their weaknesses, also make them feel in control of the situation, all in line with the vision of human-centered automation by Shneiderman [205] and others. 


\section{DisCUSSION}

It is important to say that the aforementioned add-ons can be helpful to solely solve some of the problems of today and of the nearby future. In my opinion, the farther future will require more drastic approaches in assisting the operators. This is mainly due to the continuous penetration of automation which will also become more reliable exposing less need for manual work or for constant control, as well as in fewer industrial personnel. Therefore, I imagine that in the further future, say in 100 years, one operator will be controlling an entire factory or supervising the fleet of autonomous cars of an entire city, say several hundred thousand vehicles. However, controlling larger scenes of production, one will inevitably face a different scale of data volumes as compared to today. Obviously, the need for assisting technologies that would augment operators' capabilities and enhance their work practices will be even more drastic. Therefore, I believe that the aforementioned extensions of existing interaction tools will be simply not enough to meet the growing demands. There will be a necessity for a new generation of tools and solutions that should be based on completely new approaches and principles. To achieve that, there might be a need to reconsider the current interaction methods and potentially go away from some of them, for example, abandon the traditional control station setup and use the entire space of the control room as a holistic interactive arena. 


\section{Chapter 7}

\section{In conclusion}

In the scope of my $\mathrm{PhD}$, I have been focusing on user-centered interfaces for industrial control rooms of the future. The knowledge contributions of my PhD research are elaborated first and foremost for the interaction design community. Interaction design research is the dominant topic in which my work took place, therefore, it is only natural that the achieved results resonate mainly with interaction designers. The generated knowledge is manifold. First of all, from my standpoint, I have made a valuable contribution to the body of knowledge about the industrial domain. The information that I collected during the field studies shed light on the workflows of industrial operators and the setup of industrial control rooms. In my publications, I describe control stations and their interfaces, also routines, problems, and challenges of industrial operators. Such knowledge is essential when working on industrial user-centered solutions, but it is often inaccessible for outsiders. Therefore, I believe, the knowledge that I shared could be informative and inspiring for other researchers and practitioners working in relation to the industrial domain but having no direct access to it.

Another domain where my $\mathrm{PhD}$ research contributes with some insights is the body of knowledge about running collaborative projects between the industry and academia. Being an industrial $\mathrm{PhD}$ student, 
all of my projects were such collaborations where I had to satisfy the needs of my employer, as well as meet my research goals. Based on my experience, I drew a conclusion that the "business world" is not always very welcoming to design thinking in the way it is practiced in academia. Business stakeholders tend to be rather skeptical when assessing new design ideas, especially when they are unlike anything existing in the industry at the moment. With respect to my research, this skepticism had a certain influence on my research process. Often I had to make my concept ideas more pragmatic and adjust the way of presenting them to get the green light from the stakeholders for the continuation of research. This path was thorny for me, therefore, I believe that documenting my reflections about participating in collaborative design projects between industry and academia can be helpful to other PhD students, academia and industry representatives. My main recommendation to researchers and practitioners is to pragmatically assess the "power distribution" in the upcoming collaborative project which will help to obtain expectations about feasible outcomes from the project, applicable research methods, and an appropriate form of reporting. Knowing this information is essential before getting fully engaged in a collaborative project otherwise one might end up on a journey full of surprises.

The most valuable contribution of my PhD research is a collection of artifacts contributing to a vision of an industrial control room of the future. The future that I am orienting to is not very distant, but rather a forthcoming reality that the state-of-the-art control rooms will reach within 10-15 years. In such a control room, the operator is not tied to the workstation and is not overwhelmed with a multitude of alarming screens nor forced to interact through restrictive legacy interfaces while being under constant pressure. On the contrary, the control room of the future is a comfortable environment where the information is presented in compact but informative ways, also interaction can be done through different natural modalities. The operator is engaged in the working process, they have a full understanding of the ongoing 
situation and they know that they are attending to the most urgent issue at the moment. The workstation is not only the operator's window to the production processes but also a smart assistant. The developed prototypes bear a dual value: 1) they are embodiments of ideas and concepts that were in the focus of the current research, and 2) they are examples of potential products. Reported details of the creation processes can be of interest to other researchers and practitioners, namely how the prototypes were developed, why certain decisions were made and how particular ideas were reached. The evaluation of the prototypes reveals whether the solutions were well received or rejected by the industrial personnel. This knowledge can propose future directions for research and can be taken as evidence whether the solutions should be productized.

Summarizing the work conducted, I see the overall goal of my $\mathrm{PhD}$ as a way a) to turn public attention to the high-level problems of industrial operators, b) highlight the need for assisting tools and interfaces in industrial control rooms and c) design examples of such interfaces to demonstrate their feasibility. Unfortunately, in my PhD, I did not have full control over my research process to bring all my ideas to life. However, even the works that I have implemented draw a more positive picture of the future operator work environment. I want to believe that my work will encourage others to bring design thinking into the industrial domain. By growing the catalog of user-centered industrial interfaces, a pattern will potentially emerge that would help to better comprehend and even foresee what kinds of technologies will be needed to assist the operator of the future to maintain high productivity and ensure safety in industrial production. 



\section{Bibliography}

[1] Lena Abrahamsson and Jan Johansson. "From grounded skills to sky qualifications: a study of workers creating and recreating qualifications, identity and gender at an underground iron ore mine in Sweden". In: Journal of Industrial Relations 48.5 (2006), pp. 657-676.

[2] Chadia Abras, Diane Maloney-Krichmar, Jenny Preece, et al. "User-centered design". In: Bainbridge, W. Encyclopedia of Human-Computer Interaction. Thousand Oaks: Sage Publications 37.4 (2004), pp. 445-456.

[3] Marilyn Jager Adams, Yvette J Tenney, and Richard W Pew. "Situation awareness and the cognitive management of complex systems". In: Human factors 37.1 (1995), pp. 85-104.

[4] Randall William Adams. "Peripheral vision and visual attention". In: (1971).

[5] John R Aiello and Kathryn J Kolb. “Electronic performance monitoring and social context: Impact on productivity and stress." In: Journal of Applied Psychology 80.3 (1995), p. 339.

[6] Victor Alvarez-Cortes, Gerencia De Supervisión De Procesos, Victor H Zárate Silva, Benjamin E Zayas Pérez, Jorge A Ramırez Uresti, et al. "A Personalization Approach Based on Models Integration for a User Interface for Supervision in a Power Plant". In: (2011). 
[7] Alan Baddeley. "Working memory". In: Science 255.5044 (1992), pp. 556-559.

[8] Adedeji B Badiru and Cassie Barlow. Defense Innovation Handbook: Guidelines, Strategies, and Techniques. CRC Press, 2018.

[9] Gilles Bailly, Thomas Pietrzak, Jonathan Deber, and Daniel J Wigdor. "Métamorphe: augmenting hotkey usage with actuated keys". In: Proceedings of the SIGCHI Conference on Human Factors in Computing Systems. ACM. 2013, pp. 563-572.

[10] Gilles Bailly, Sidharth Sahdev, Sylvain Malacria, and Thomas Pietrzak. "LivingDesktop: Augmenting desktop workstation with actuated devices". In: Proceedings of the 2016 CHI Conference on Human Factors in Computing Systems. ACM. 2016, pp. 5298-5310.

[11] Lisanne Bainbridge. "Ironies of automation". In: Analysis, design and evaluation of man-machine systems. Elsevier, 1983, pp. 129-135.

[12] Till Ballendat, Nicolai Marquardt, and Saul Greenberg. "Proxemic interaction: designing for a proximity and orientation-aware environment". In: ACM International Conference on Interactive Tabletops and Surfaces. ACM. 2010, pp. 121-130.

[13] Mark Baskinger and Mark Gross. "Cover story tangible interaction $=$ form + computing". In: interactions 17.1 (2010), pp. 6-11.

[14] Patrick Baudisch, Desney Tan, Maxime Collomb, Dan Robbins, Ken Hinckley, Maneesh Agrawala, Shengdong Zhao, and Gonzalo Ramos. "Phosphor: explaining transitions in the user interface using afterglow effects". In: Proceedings of the 19th annual ACM symposium on User interface software and technology. 2006, pp. 169-178. 
[15] Gordon Baxter, John Rooksby, Yuanzhi Wang, and Ali Khajeh-Hosseini. "The ironies of automation: still going strong at 30?" In: Proceedings of the 30th European Conference on Cognitive Ergonomics. 2012, pp. 65-71.

[16] Andrew H Bellenkes, Christopher D Wickens, and Arthur F Kramer. "Visual scanning and pilot expertise: the role of attentional flexibility and mental model development." In: Aviation, space, and environmental medicine (1997).

[17] Daniel E Berlyne. "Conflict, arousal, and curiosity." In: (1960).

[18] Kent C Berridge and Terry E Robinson. "What is the role of dopamine in reward: hedonic impact, reward learning, or incentive salience?" In: Brain research reviews 28.3 (1998), pp. 309-369.

[19] Jacques Bertin. "Semiology of graphics (WJ Berg, Trans.)" In: Madison, WI: The University of Wisconsin Press.(Original work published 1967) (1983).

[20] Enrico Bertini and Denis Lalanne. "Surveying the complementary role of automatic data analysis and visualization in knowledge discovery". In: Proceedings of the ACM SIGKDD Workshop on Visual Analytics and Knowledge Discovery: Integrating Automated Analysis with Interactive Exploration. 2009, pp. 12-20.

[21] Nigel Bevan. "Criteria for Selecting Methods in User Centred Design." In: I-USED. Citeseer. 2009.

[22] S James Biggs and Mandayam A Srinivasan. "Haptic interfaces". In: Handbook of virtual environments. CRC Press, 2002, pp. 133-156.

[23] Charles E Billings. "Human-centered aircraft automation: A concept and guidelines". In: (1991). 
[24] Florian Block, Hans Gellersen, and Nicolas Villar. "Touchdisplay keyboards: transforming keyboards into interactive surfaces". In: Proceedings of the SIGCHI Conference on Human Factors in Computing Systems. ACM. 2010, pp. 1145-1154.

[25] Fritz Böhle and Brigitte Milkau. "Computerised manufacturing and empirical knowledge". In: AI \& SOCIETY 2.3 (1988), pp. 235-243.

[26] Charles F Bond and Linda J Titus. "Social facilitation: a metaanalysis of 241 studies." In: Psychological bulletin 94.2 (1983), p. 265.

[27] John Bowers. "The logic of annotated portfolios: communicating the value of'research through design'". In: Proceedings of the Designing Interactive Systems Conference. ACM. 2012, pp. 68-77.

[28] DE Broadbent. Perception and communication. Elmsford, NY, US. 1958.

[29] Donald Eric Broadbent. “Decision and stress.” In: (1971).

[30] Donald Eric Broadbent. Perception and communication. Elsevier, 2013.

[31] Alex AT Bui and William Hsu. "Medical data visualization: Toward integrated clinical workstations". In: Medical Imaging Informatics. Springer, 2010, pp. 139-193.

[32] HJ Bullinger and HJ Warnecke. "Toward the Factory of the Future". In: Toward the Factory of the Future: Proceedings of the 8th International Conference on Production Research and 5th Working Conference of the Fraunhofer-Institute for Industrial Engineering (FHG-IAO) at University of Stuttgart, August 20-22, 1985. Springer Verlag. 1985, p. 133.

[33] Grigore C Burdea and Philippe Coiffet. Virtual reality technology. John Wiley \& Sons, 2003. 
[34] Jennifer L Burke, Robin R Murphy, Michael D Coovert, and Dawn L Riddle. "Moonlight in Miami: Field study of humanrobot interaction in the context of an urban search and rescue disaster response training exercise". In: Human-Computer Interaction 19.1-2 (2004), pp. 85-116.

[35] Alfred Dupont Chandler, Takashi Hikino, and Alfred D Chandler. Scale and scope: The dynamics of industrial capitalism. Harvard University Press, 2009.

[36] Bay-Wei Chang and David Ungar. "Animation: from cartoons to the user interface". In: Proceedings of the 6th annual ACM symposium on User interface software and technology. 1993, pp. 45-55.

[37] Alphonse Chapanis. "On the allocation of functions between men and machines". In: Occupational Psychology 39.1 (1965), pp. 1-11.

[38] CCPS (Center for Chemical Process Safety) and American Institute of Chemical Engineers. Center for Chemical Process Safety. Guidelines for Safe Automation of Chemical Processes. John Wiley \& Sons, 2016.

[39] CL Philip Chen and Chun-Yang Zhang. "Data-intensive applications, challenges, techniques and technologies: A survey on Big Data". In: Information Sciences 275 (2014), pp. 314347.

[40] EHC Choi, R Taib, Y Shi, and F Chen. "Multimodal user interface for traffic incident management in control room". In: IET Intelligent Transport Systems 1.1 (2007), pp. 27-36.

[41] Jaegul Choo, Hanseung Lee, Zhicheng Liu, John Stasko, and Haesun Park. "An interactive visual testbed system for dimension reduction and clustering of large-scale highdimensional data". In: Visualization and Data Analysis 2013. Vol. 8654. International Society for Optics and Photonics. 2013, p. 865402. 
[42] Michael A Cohen, Patrick Cavanagh, Marvin M Chun, and Ken Nakayama. "The attentional requirements of consciousness". In: Trends in cognitive sciences 16.8 (2012), pp. 411-417.

[43] National Research Council et al. The future of air traffic control: Human operators and automation. National Academies Press, 1998.

[44] Nelson Cowan. "The magical number 4 in short-term memory: A reconsideration of mental storage capacity". In: Behavioral and brain sciences 24.1 (2001), pp. 87-114.

[45] Mihaly Csikszentmihalyi and Isabella Selega Csikszentmihalyi. Optimal experience: Psychological studies of flow in consciousness. Cambridge university press, 1992.

[46] Mary Cummings. "Automation bias in intelligent time critical decision support systems". In: AIAA 1st Intelligent Systems Technical Conference. 2004, p. 6313.

[47] Bruce H Deatherage. "Auditory and other sensory forms of information presentation". In: Human engineering guide to equipment design (1972), pp. 123-160.

[48] J Anthony Deutsch and Diana Deutsch. "Attention: Some theoretical considerations." In: Psychological review 70.1 (1963), p. 80.

[49] Paul H Dietz, Benjamin D Eidelson, Jonathan Westhues, and Steven Bathiche. "A practical pressure sensitive computer keyboard." In: UIST. Vol. 9. 2009, pp. 55-58.

[50] Veronika Domova. "Fidgeting with the Environment: a Tangible Control for Interacting with a Smart Light". Submitted.

[51] Veronika Domova. "Guiding the Operator's Attention Among a Plurality of Operator Workstation Screens". Submitted. 
[52] Veronika Domova, Saad Azhar, Maria Ralph, and Jonas Brönmark. “Untethered Workspaces: A Zones Concept Towards Supporting Operator Movements in Control Rooms". In: Proceedings of the 2016 CHI Conference Extended Abstracts on Human Factors in Computing Systems. ACM. 2016, pp. 680689.

[53] Veronika Domova and Aldo Dagnino. "Towards intelligent alarm management in the Age of IIoT". In: 2017 Global Internet of Things Summit (GIoTS). IEEE. 2017, pp. 1-5.

[54] Veronika Domova, Erik Gärtner, Johan Källström, Martin Pallin, Fredrik Präntare, and Nikita Korzhitskii. “Improving Usability of Decision Support Systems for SAR Operations: WARA-PS case study". Submitted.

[55] Veronika Domova, Alvaro Aranda Munoz, Elsa Vaara, and Petra Edoff. "Feel the Water: Expressing Physicality of District Heating Processes in Functional Overview Displays". In: ACM International Conference on Interactive Surfaces and Spaces. ACM. 2019.

[56] Veronika Domova, Maria Ralph, Elina Vartiainen, Alvaro Aranda Muñoz, Adam Henriksson, and Susanne Timsjö. "Re-Introducing Physical User Interfaces into Industrial Control Rooms". In: Proceedings of the European Conference on Cognitive Ergonomics 2017. ACM. 2017, pp. 162-168.

[57] Veronika Domova and Shiva Sander-Tavallaey. "Visualization for quality health-care: patient flow exploration". In: IEEE International Conference on Big Data. IEEE. 2019.

[58] Veronika Domova, Elina Vartiainen, Saad Azhar, and Maria Ralph. "An interactive surface solution to support collaborative work onboard ships". In: Proceedings of the 2013 ACM international conference on Interactive tabletops and surfaces. ACM. 2013, pp. 265-272. 
[59] Veronika Domova, Elina Vartiainen, and Marcus Englund. "Designing a remote video collaboration system for industrial settings". In: Proceedings of the Ninth ACM International Conference on Interactive Tabletops and Surfaces. ACM. 2014, pp. 229-238.

[60] Veronika Domova and Katerina Vrotsou. "A Model for Types and Levels of Automation in Visual Analytics: Examples from Event-Sequence Analytics". Manuscript in preparation.

[61] Veronika Domova and Goranka Zoric. “Towards Effective Industrial Robot Fleet Visualization for Remote Service Applications". In: Enabling Technologies: Infrastructure for Collaborative Enterprises (WETICE), 2017 IEEE 26th International Conference on. IEEE. 2017, pp. 185-190.

[62] Paul Dourish. Where the action is. MIT press Cambridge, 2001.

[63] Francis T Durso and Scott D Gronlund. "Situation awareness". In: Handbook of applied cognition (1999), pp. 283-314.

[64] Kenneth D Eason. Information technology and organisational change. CRC Press, 1989.

[65] Elwyn Edwards and Frank P Lees. Man and computer in process control. Institution of Chemical Engineers, 1973.

[66] EEMUA. “EEMUA-191:Engineering Equipment and Materials Users' Association". In: Alarm Systems: A Guide to Design, Management and Procurement, Second Edition. London (2007).

[67] "Effects of age and instructions on teaching older adults to use Eldercomm, an electronic bulletin board system". In: Educational Gerontology 26.3 (2000), pp. 221-235.

[68] Mica Endsley and William M Jones. Situation Awareness Information Dominance \& Information Warfare. Tech. rep. LOGICON TECHNICAL SERVICES INC DAYTON OH, 1997. 
[69] Mica R Endsley. "Level of automation effects on performance, situation awareness and workload in a dynamic control task". In: Ergonomics 42.3 (1999), pp. 462-492.

[70] Mica R Endsley. "The application of human factors to the development of expert systems for advanced cockpits". In: Proceedings of the Human Factors Society Annual Meeting. Vol. 31. 12. SAGE Publications Sage CA: Los Angeles, CA. 1987, pp. 1388-1392.

[71] Mica R Endsley et al. "The role of situation awareness in naturalistic decision making". In: Naturalistic decision making 269 (1997), p. 284.

[72] Mica R Endsley. "Toward a theory of situation awareness in dynamic systems". In: Situational Awareness. Routledge, 2017, pp. 9-42.

[73] Mica R Endsley and Esin O Kiris. "The out-of-the-loop performance problem and level of control in automation". In: Human factors 37.2 (1995), pp. 381-394.

[74] Johan Fagerlönn, Kristin Hammarberg, Stefan Lindberg, Anna Sirkka, and Sofia Larsson. "Designing a Multimodal Warning Display for an Industrial Control Room". In: Proceedings of the 12th International Audio Mostly Conference on Augmented and Participatory Sound and Music Experiences. ACM. 2017, p. 46.

[75] Karen M Feigh and Amy R Pritchett. "Requirements for effective function allocation: A critical review". In: Journal of cognitive engineering and decision making 8.1 (2014), pp. 23-32.

[76] Stephen Few. "Information dashboard design". In: (2006).

[77] Paul M Fitts. "Human engineering for an effective airnavigation and traffic-control system." In: (1951).

[78] Paul M Fitts and Michael I Posner. "Human performance." In: (1967). 
[79] Terrence Fong, Charles Thorpe, and Charles Baur. Collaborative control: A robot-centric model for vehicle teleoperation. Vol. 1. Carnegie Mellon University, The Robotics Institute Pittsburgh, 2001.

[80] Christopher Frayling. "Research in art and design". In: (1993).

[81] Ronja Frimalm, Johan Fagerlönn, Stefan Lindberg, and Anna Sirkka. "How Many Auditory Icons, in a Control Room Environment, Can You Learn?" In: Georgia Institute of Technology. 2014.

[82] Jörgen Frohm, Veronica Lindström, Johan Stahre, and Mats Winroth. "Levels of automation in manufacturing". In: Ergonomia-an International journal of ergonomics and human factors 30.3 (2008).

[83] Ferdinand Fuhrmann and Rene Kaiser. "Multimodal Interaction for Future Control Centers: An Interactive Demonstrator". In: Proceedings of the 16th International Conference on Multimodal Interaction. ACM. 2014, pp. 66-67.

[84] David M Gaba, Steven K Howard, and Stephen D Small. "Situation awareness in anesthesiology". In: Human factors 37.1 (1995), pp. 20-31.

[85] Bill Gaver and John Bowers. "Annotated portfolios". In: interactions 19.4 (2012), pp. 40-49.

[86] William Gaver. "What should we expect from research through design?" In: Proceedings of the SIGCHI conference on human factors in computing systems. 2012, pp. 937-946.

[87] James J Gibson. The ecological approach to visual perception: classic edition. Psychology Press, 2014.

[88] James J Gibson. "The perception of the visual world." In: (1950). 
[89] Robert Glaser. "Expertise and learning: How do we think about instructional processes now that we have discovered knowledge structures". In: Complex information processing: The impact of Herbert A. Simon (1989), pp. 269-282.

[90] John D Gould, Stephen J Boies, and Clayton Lewis. "Making usable, useful, productivity-enhancing computer applications". In: Communications of the ACM 34.1 (1991), pp. 7485 .

[91] John D Gould and Clayton Lewis. "Designing for usability: key principles and what designers think". In: Communications of the ACM 28.3 (1985), pp. 300-311.

[92] Camilla Grane, Lena Abrahamsson, Jonas Andersson, Cecilia Berlin, Åsa Fasth, Jan Johansson, Johan Stahre, and Anna-Lisa Osvalder. "The operator of the future-a key to competitive industry in a future information society". In: Swedish Production Symposium: 06/11/2012-08/11/2012. 2012.

[93] Mikell P Groover. "Automation, Production Systems, and Computer-integrated Manufacturing 2nd ed." In: Assembly automation (2002).

[94] JoAnn T Hackos and Janice Redish. User and task analysis for interface design. Vol. 1. Wiley New York, 1998.

[95] Kelly S Hale and Kay M Stanney. "Deriving haptic design guidelines from human physiological, psychophysical, and neurological foundations". In: IEEE computer graphics and applications 24.2 (2004), pp. 33-39.

[96] Edward Twitchell Hall. The hidden dimension. Vol. 609. Garden City, NY: Doubleday, 1966.

[97] Sung H Han, Huichul Yang, and Dong-Gwan Im. “Designing a human-computer interface for a process control room: A case study of a steel manufacturing company". In: International Journal of Industrial Ergonomics 37.5 (2007), pp. 383393. 
[98] PA Hancock and SF Scallen. "The future of function allocation". In: Ergonomics in design 4.4 (1996), pp. 24-29.

[99] Cristina Handal and Laura H Ikuma. “Good Interface Design Improves Situation Awareness in Control Room Operators". In: IIE Annual Conference. Proceedings. Institute of Industrial and Systems Engineers (IISE). 2012, p. 1.

[100] Rex Hartson and Pardha S Pyla. The UX Book: Process and guidelines for ensuring a quality user experience. Elsevier, 2012.

[101] Reza Hassanzadeh and Zorica Nedovic-Budic. "Where to go first: prioritization of damaged areas for allocation of Urban Search and Rescue (USAR) operations (PI-USAR model)". In: Geomatics, Natural Hazards and Risk 7.4 (2016), pp. 13371366.

[102] Tomi Heimonen, Jaakko Hakulinen, Sumita Sharma, Markku Turunen, Lauri Lehtikunnas, and Hannu Paunonen. "Multimodal interaction in process control rooms: are we there yet?" In: Proceedings of the 5th ACM International Symposium on Pervasive Displays. ACM. 2016, pp. 20-32.

[103] Eva Heiskanen, Kaarina Hyvönen, Mari Niva, Mika Pantzar, Päivi Timonen, and Johanna Varjonen. "User involvement in radical innovation: are consumers conservative?" In: European Journal of Innovation Management (2007).

[104] Valentin Heun, Anette von Kapri, and Pattie Maes. "Perifoveal display: combining foveal and peripheral vision in one visualization". In: Proceedings of the 2012 ACM Conference on Ubiquitous Computing. ACM. 2012, pp. 1150-1155.

[105] Clint Heyer and Kristoffer Husøy. "Interaction with the dirty, dangerous, and dull". In: interactions 19.4 (2012), pp. 19-23.

[106] Mads Hobye, Jonas Löwgren, and Henrik Svarrer LARSEN. "Towards programmatic design research". In: Designs for learning 6.1-2 (2013), pp. 80-100. 
[107] Erik Hollnagel. "From function allocation to function congruence". In: Coping with computers in the cockpit $(A$ 00-40958 11-54), Aldershot, United Kingdom and Brookfield, VT, Ashgate Publishing, 1999, (1999), pp. 29-53.

[108] Magnus Holm, Göran Adamson, Lihui Wang, and Philip Moore. "The future Swedish shop-floor operator-interviews with production managers". In: The sixth Swedish Production Symposium, 2014, September 16-18, Gothenburg. 2014.

[109] Karen Holtzblatt and Hugh Beyer. Contextual design: defining customer-centered systems. Elsevier, 1997.

[110] Kristina Höök and Jonas Löwgren. "Strong concepts: Intermediate-level knowledge in interaction design research". In: ACM Transactions on Computer-Human Interaction (TOCHI) 19.3 (2012), pp. 1-18.

[111] Wenkai Hu, Muhammad Shahzad Afzal, Gustavo Brandt, Eric Lau, Tongwen Chen, and Sirish L Shah. "An application of advanced alarm management tools to an oil sand extraction plant". In: IFAC-PapersOnLine 48.8 (2015), pp. 641-646.

[112] Ira E Hyman Jr, S Matthew Boss, Breanne M Wise, Kira E McKenzie, and Jenna M Caggiano. “Did you see the unicycling clown? Inattentional blindness while walking and talking on a cell phone". In: Applied Cognitive Psychology 24.5 (2010), pp. 597-607.

[113] A Inselberg and B Dimsdale. Parallel Coordinates: A Tool for Visualizing Multivariate Relations (chapter 9). 1991.

[114] ANSI ISA. "ISA-18.2: Management of Alarm Systems for the Process Industries". In: International Society of Automation. Durham, NC, USA (2009).

[115] S5 ISA. Instrumentation symbols and identification. 1992.

[116] ISO13407 ISO. "13407: Human-centred design processes for interactive systems". In: Geneva: ISO (1999). 
[117] Sheena Iyengar. The art of choosing. Twelve, 2010.

[118] Sheena S Iyengar and Mark R Lepper. "When choice is demotivating: Can one desire too much of a good thing?" In: Journal of personality and social psychology 79.6 (2000), p. 995.

[119] Mads Vedel Jensen and Marcelle Stienstra. "Making sense: Interactive sculptures as tangible design material". In: Proceedings of the 2007 conference on Designing pleasurable products and interfaces. ACM. 2007, pp. 255-269.

[120] G Johannsen. "Conceptual design of multi-human machine interfaces". In: Control Engineering Practice 5.3 (1997), pp. 349-361.

[121] Kenneth O Johnson and Takashi Yoshioka. Neural mechanisms of tactile form and texture perception. na, 2002.

[122] Matthew Johnson, Jeffrey M Bradshaw, and Paul J Feltovich. "Tomorrow's human-machine design tools: From levels of automation to interdependencies". In: Journal of Cognitive Engineering and Decision Making 12.1 (2018), pp. 77-82.

[123] Matthew Johnson, Jeffrey M Bradshaw, Paul J Feltovich, Catholijn M Jonker, Birna Van Riemsdijk, and Maarten Sierhuis. "The fundamental principle of coactive design: Interdependence must shape autonomy". In: International Workshop on coordination, organizations, institutions, and norms in agent systems. Springer. 2010, pp. 172-191.

[124] Wolfgang Jonas. "Design Research and its Meaning to the Methodological Development of the Discipline". In: Design research now (2007), pp. 187-206.

[125] Debra G Jones and Mica R Endsley. "Sources of situation awareness errors in aviation." In: Aviation, space, and environmental medicine (1996). 
[126] Nehemiah Jordan. "Allocation of functions between man and machines in automated systems." In: Journal of applied psychology 47.3 (1963), p. 161.

[127] David B Kaber and Mica R Endsley. “The combined effect of level of automation and adaptive automation on human performance with complex, dynamic control systems". In: Proceedings of the Human Factors and Ergonomics Society Annual Meeting. Vol. 41. 1. SAGE Publications Sage CA: Los Angeles, CA. 1997, pp. 205-209.

[128] D Kahneman. “Think Fast, Think Slow". In: Farrar, Straus and Giroux, New York (2011).

[129] Daniel Kahneman. Attention and effort. Vol. 1063. Citeseer, 1973.

[130] Neung Eun Kang and Wan Chul Yoon. "Age-and experiencerelated user behavior differences in the use of complicated electronic devices". In: International journal of humancomputer studies 66.6 (2008), pp. 425-437.

[131] Matti A Kaulio. “Customer, consumer and user involvement in product development: A framework and a review of selected methods". In: Total quality management 9.1 (1998), pp. 141-149.

[132] K Kawai. "An intelligent multimedia human interface for highly automated combined-cycle plants". In: Control Engineering Practice 5.3 (1997), pp. 401-406.

[133] Daniel Keim, Gennady Andrienko, Jean-Daniel Fekete, Carsten Görg, Jörn Kohlhammer, and Guy Melançon. "Visual analytics: Definition, process, and challenges". In: Information visualization. Springer, 2008, pp. 154-175.

[134] Daniel Keim, Jörn Kohlhammer, Geoffrey Ellis, and Florian Mansmann. "Mastering the information age: solving problems with visual analytics". In: (2010). 
[135] Daniel A Keim, H-P Kriegel, and Mihael Ankerst. “Recursive pattern: A technique for visualizing very large amounts of data". In: Proceedings Visualization'95. IEEE. 1995, pp. 279286.

[136] Horst Kern and Michael Schumann. Industriearbeit und Arbeiterbewußtsein: eine empirische Untersuchung über den Einfluß der aktuellen technischen Entwicklung auf die industrielle Arbeit und das Arbeiterbewußtsein. Vol. 1. Europäische Verlagsanstalt, 1974.

[137] Alicia Key, Bill Howe, Daniel Perry, and Cecilia Aragon. "Vizdeck: self-organizing dashboards for visual analytics". In: Proceedings of the 2012 ACM SIGMOD International Conference on Management of Data. ACM. 2012, pp. 681-684.

[138] Hyunyoung Kim, Patrıcia Deud Guimarães, Céline Coutrix, and Anne Roudaut. "ExpanDial: Designing a ShapeChanging Dial". In: Proceedings of the 2019 on Designing Interactive Systems Conference. ACM. 2019, pp. 949-961.

[139] Lawrence H Kim and Sean Follmer. “Ubiswarm: Ubiquitous robotic interfaces and investigation of abstract motion as a display". In: Proceedings of the ACM on Interactive, Mobile, Wearable and Ubiquitous Technologies 1.3 (2017), p. 66.

[140] Lawrence H. Kim, Daniel S. Drew, Veronika Domova, and Sean Follmer. "User-Defined Swarm Robot Control". In: Proceedings of the 2020 CHI Conference on Human Factors in Computing Systems. CHI '20. Honolulu, HI, USA: Association for Computing Machinery, 2020, pp. 1-13.

[141] Seoktae Kim, Hyunjung Kim, Boram Lee, Tek-Jin Nam, and Woohun Lee. "Inflatable mouse: volume-adjustable mouse with air-pressure-sensitive input and haptic feedback". In: Proceedings of the SIGCHI Conference on Human Factors in Computing Systems. ACM. 2008, pp. 211-224. 
[142] Francisco Kiss, Valentin Schwind, Stefan Schneegass, and Niels Henze. "Design and evaluation of a computer-actuated mouse". In: Proceedings of the 16th International Conference on Mobile and Ubiquitous Multimedia. ACM. 2017, pp. 261-271.

[143] Glen Klien, David D Woods, Jeffrey M Bradshaw, Robert R Hoffman, and Paul J Feltovich. "Ten challenges for making automation a" team player" in joint human-agent activity". In: IEEE Intelligent Systems 19.6 (2004), pp. 91-95.

[144] Annette Kluge, Mike Silbert, Uta S Wiemers, Barbara Frank, and Oliver T Wolf. "Retention of a standard operating procedure under the influence of social stress and refresher training in a simulated process control task". In: Ergonomics 62.3 (2019), pp. 361-375.

[145] Sheldon J Korchin. "Anxiety and cognition". In: Cognition: Theory, research, promise. New York: Harper \& Row (1964), pp. 58-78.

[146] Ilpo Koskinen, John Zimmerman, Thomas Binder, Johan Redstrom, and Stephan Wensveen. Design research through practice: From the lab, field, and showroom. Elsevier, 2011.

[147] Hiroyuki Kudo, Koichi Suzumori, and Takefumi Kanda. "Force feedback mouse with differential mechanism for omni-traveling". In: 2007 International Symposium on MicroNanoMechatronics and Human Science. IEEE. 2007, pp. 430435 .

[148] Yoshiki Kudo, Kazuki Takashima, Morten Fjeld, and Yoshifumi Kitamura. "AdapTable: Extending Reach over Large Tabletops Through Flexible Multi-Display Configuration". In: Proceedings of the 2018 ACM International Conference on Interactive Surfaces and Spaces. ACM. 2018, pp. 213-225. 
[149] Tatiana von Landesberger, Sebastian Fiebig, Sebastian Bremm, Arjan Kuijper, and Dieter W Fellner. "Interaction taxonomy for tracking of user actions in visual analytics applications". In: Handbook of Human Centric Visualization. Springer, 2014, pp. 653-670.

[150] Adam M Larson and Lester C Loschky. "The contributions of central versus peripheral vision to scene gist recognition". In: Journal of Vision 9.10 (2009), pp. 6-6.

[151] Jean Lave and Etienne Wenger. Situated learning: Legitimate peripheral participation. Cambridge university press, 1991.

[152] Bokyung Lee, Sindy $\mathrm{Wu}$, Maria Jose Reyes, and Daniel Saakes. "The Effects of Interruption Timings on Autonomous Height-Adjustable Desks that Respond to Task Changes". In: Proceedings of the 2019 CHI Conference on Human Factors in Computing Systems. ACM. 2019, p. 328.

[153] Youn-Kyung Lim, Erik Stolterman, and Josh Tenenberg. "The anatomy of prototypes: Prototypes as filters, prototypes as manifestations of design ideas". In: ACM Transactions on Computer-Human Interaction (TOCHI) 15.2 (2008), p. 7.

[154] Lars Lischke, Paweł W Woźniak, Sven Mayer, Andreas Preikschat, and Morten Fjeld. "Using Variable Movement Resistance Sliders for Remote Discrete Input". In: Proceedings of the 2017 ACM International Conference on Interactive Surfaces and Spaces. ACM. 2017, pp. 116-125.

[155] Jonas Löwgren. "Annotated portfolios and other forms of intermediate-level knowledge". In: interactions 20.1 (2013), pp. 30-34.

[156] Jonas Löwgren. "On the significance of making in interaction design research". In: interactions 23.3 (2016), pp. 26-33. 
[157] Steven J Luck and Edward K Vogel. "Visual working memory capacity: from psychophysics and neurobiology to individual differences". In: Trends in cognitive sciences 17.8 (2013), pp. 391-400.

[158] I Scott MacKenzie, R William Soukoreff, and Chris Pal. "A two-ball mouse affords three degrees of freedom". In: $\mathrm{CHI}^{\prime} 97$ Extended Abstracts on Human Factors in Computing Systems. ACM. 1997, pp. 303-304.

[159] Dariusz Man and Ryszard Olchawa. "The Possibilities of Using BCI Technology in Biomedical Engineering". In: International Scientific Conference BCI 2018 Opole. Springer. 2018, pp. 30-37.

[160] Peter Mathias. The first industrial nation: The economic history of Britain 1700-1914. Routledge, 2013.

[161] Neville Moray. "The role of attention in the detection of errors and the diagnosis of failures in man-machine systems". In: Human detection and diagnosis of system failures. Springer, 1981, pp. 185-198.

[162] Neville Moray, Toshiyuki Inagaki, and Makoto Itoh. “Adaptive automation, trust, and self-confidence in fault management of time-critical tasks." In: Journal of experimental psychology: Applied 6.1 (2000), p. 44.

[163] D Mourtzis, E Vlachou, and N Milas. “Industrial Big Data as a result of IoT adoption in manufacturing". In: Procedia CIRP 55 (2016), pp. 290-295.

[164] Jens Müller, Tobias Schwarz, Simon Butscher, and Harald Reiterer. "Back to tangibility: a post-WIMP perspective on control room design". In: Proceedings of the 2014 International Working Conference on Advanced Visual Interfaces. ACM. 2014, pp. 57-64.

[165] Jaap MJ Murre and Joeri Dros. "Replication and analysis of Ebbinghaus' forgetting curve". In: PloS one 10.7 (2015). 
[166] Paul W Murrill. Fundamentals of process control theory. Instrument Society of America Research Triangle Park, NC, 1991.

[167] Ken Nakagaki, Daniel Fitzgerald, Zhiyao John Ma, Luke Vink, Daniel Levine, and Hiroshi Ishii. "inFORCE: BidirectionalForce'Shape Display for Haptic Interaction". In: Proceedings of the Thirteenth International Conference on Tangible, Embedded, and Embodied Interaction. ACM. 2019, pp. 615623.

[168] Randall J Nelson. The somatosensory system: Deciphering the brain's own body image. CRC Press, 2001.

[169] Jakob Nielsen. Usability engineering. Morgan Kaufmann, 1994.

[170] Benjamin Noah, Jung-Hyup Kim, Ling Rothrock, and Anand Tharanathan. "Evaluating alternate visualization techniques for overview displays in process control". In: IIE Transactions on Occupational Ergonomics and Human Factors 2.3-4 (2014), pp. 152-168.

[171] Don Norman. The design of everyday things: Revised and expanded edition. Basic books, 2013.

[172] Donald A Norman. "The 'problem'with automation: inappropriate feedback and interaction, not 'over-automation'". In: Philosophical Transactions of the Royal Society of London. B, Biological Sciences 327.1241 (1990), pp. 585-593.

[173] Donald A Norman. "THE WAY I SEE IT Systems thinking: a product is more than the product". In: Interactions 16.5 (2009), pp. 52-54.

[174] Donald A Norman and Stephen W Draper. User centered system design: New perspectives on human-computer interaction. CRC Press, 1986. 
[175] Diana Nowacka, Katrin Wolf, Enrico Costanza, and David Kirk. "Working with an Autonomous Interface: Exploring the Output Space of an Interactive Desktop Lamp". In: Proceedings of the Twelfth International Conference on Tangible, Embedded, and Embodied Interaction. ACM. 2018, pp. 1-10.

[176] Linda Onnasch, Christopher D Wickens, Huiyang Li, and Dietrich Manzey. "Human performance consequences of stages and levels of automation: An integrated metaanalysis". In: Human factors 56.3 (2014), pp. 476-488.

[177] Stephen Palmer and Irvin Rock. "Rethinking perceptual organization: The role of uniform connectedness". In: Psychonomic bulletin \& review 1.1 (1994), pp. 29-55.

[178] Raja Parasuraman, Thomas B Sheridan, and Christopher D Wickens. "A model for types and levels of human interaction with automation". In: IEEE Transactions on systems, man, and cybernetics-Part A: Systems and Humans 30.3 (2000), pp. 286297.

[179] Wanjoo Park, Sehyung Park, Laehyun Kim, and Seungjae Shin. "Haptic mouse interface actuated by an electromagnet". In: 2011 International Conference on Complex, Intelligent, and Software Intensive Systems. IEEE. 2011, pp. 643-646.

[180] Gary Perelman, Marcos Serrano, Mathieu Raynal, Celia Picard, Mustapha Derras, and Emmanuel Dubois. "The rolypoly mouse: Designing a rolling input device unifying $2 \mathrm{~d}$ and 3d interaction". In: Proceedings of the 33rd Annual ACM Conference on Human Factors in Computing Systems. ACM. 2015, pp. 327-336.

[181] Daniel H Pink. Drive: The surprising truth about what motivates us. Penguin, 2011.

[182] James R Pomerantz, Michael Kubovy, et al. “Theoretical approaches to perceptual organization: Simplicity and likelihood principles". In: Organization 36.3 (1986), pp. 36-1. 
[183] Pere Ponsa Asensio, Beatriz Amante Garcia, and Marta Diaz Boladeras. "Ergonomic design applied in a sugar mill interface". In: Latin American applied research 40.1 (2010), pp. 2733.

[184] Hamid Reza Ranjbar, Alireza A Ardalan, Hamid Dehghani, and Mohammad Reza Saradjian. "A proposed spatial index to prioritize damaged buildings for allocating USAR operations". In: Geocarto international 33.8 (2018), pp. 791-809.

[185] Jens Rasmussen. "Models of mental strategies in process plant diagnosis". In: Human detection and diagnosis of system failures. Springer, 1981, pp. 241-258.

[186] James Reason. Human error. Cambridge university press, 1990.

[187] Dal Vernon C Reising and Peter T Bullemer. "A direct perception, span-of-control overview display to support a process control operator's situation awareness: A practiceoriented design process". In: Proceedings of the Human Factors and Ergonomics Society Annual Meeting. Vol. 52. 4. SAGE Publications Sage CA: Los Angeles, CA. 2008, pp. 267-271.

[188] Patrick Renner and Thies Pfeiffer. "Attention guiding techniques using peripheral vision and eye tracking for feedback in augmented-reality-based assistance systems". In: 2017 IEEE Symposium on 3D User Interfaces (3DUI). IEEE. 2017, pp. 186-194.

[189] Ronald A Rensink, J Kevin O'Regan, and James J Clark. “To see or not to see: The need for attention to perceive changes in scenes". In: Psychological science 8.5 (1997), pp. 368-373.

[190] Sandy Ressler, Brian Antonishek, Qiming Wang, and Afzal Godil. "Integrating active tangible devices with a synthetic environment for collaborative engineering". In: Proceedings of the sixth international conference on 3D Web technology. ACM. 2001, pp. 93-100. 
[191] Jennifer M Riley and Mica R Endsley. "The hunt for situation awareness: Human-robot interaction in search and rescue". In: Proceedings of the Human Factors and Ergonomics Society Annual Meeting. Vol. 48. 3. SAGE Publications Sage CA: Los Angeles, CA. 2004, pp. 693-697.

[192] Eduardo Salas, Carolyn Prince, David P Baker, and Lisa Shrestha. "Situation awareness in team performance: Implications for measurement and training". In: Human factors 37.1 (1995), pp. 123-136.

[193] Farzan Sasangohar, Stacey D Scott, and Mary L Cummings. "Supervisory-level interruption recovery in timecritical control tasks". In: Applied ergonomics 45.4 (2014), pp. 1148-1156.

[194] Juergen Sauer, Sven Schmutz, Andreas Sonderegger, and Nadine Messerli. "Social stress and performance in humanmachine interaction: a neglected research field". In: Ergonomics 62.11 (2019), pp. 1377-1391.

[195] Grégory Savioz, Miroslav Markovic, and Yves Perriard. "Towards multi-finger haptic devices: A computer keyboard with adjustable force feedback". In: 2011 International Conference on Electrical Machines and Systems. IEEE. 2011, pp. 16.

[196] Mark W Scerbo. “Theoretical perspectives on adaptive automation". In: (1996).

[197] Douglas Schuler and Aki Namioka. Participatory design: Principles and practices. CRC Press, 1993.

[198] Jason M Scimeca and Steven L Franconeri. "Selecting and tracking multiple objects". In: Wiley Interdisciplinary Reviews: Cognitive Science 6.2 (2015), pp. 109-118. 
[199] Angelia Sebok and Christopher D Wickens. "Implementing lumberjacks and black swans into model-based tools to support human-automation interaction". In: Human factors 59.2 (2017), pp. 189-203.

[200] Thomas B Sheridan. "Function allocation: algorithm, alchemy or apostasy?" In: International Journal of HumanComputer Studies 52.2 (2000), pp. 203-216.

[201] Thomas B Sheridan. "Human centered automation: oxymoron or common sense?" In: 1995 IEEE International Conference on Systems, Man and Cybernetics. Intelligent Systems for the 21st Century. Vol. 1. IEEE. 1995, pp. 823-828.

[202] Thomas B Sheridan and William L Verplank. Human and computer control of undersea teleoperators. Tech. rep. Massachusetts Inst of Tech Cambridge Man-Machine Systems Lab, 1978.

[203] CE SHERRIC. "Cutaneous sensitivity". In: Handbook of perception and human performance (1986).

[204] Joon-Gi Shin, Eiji Onchi, Maria Jose Reyes, Junbong Song, Uichin Lee, Seung-Hee Lee, and Daniel Saakes. "Slow Robots for Unobtrusive Posture Correction". In: Proceedings of the 2019 CHI Conference on Human Factors in Computing Systems. ACM. 2019, p. 613.

[205] Ben Shneiderman. "Human-Centered Artificial Intelligence: Trusted, Reliable \& Safe". In: arXiv preprint arXiv:2002.04087 (2020).

[206] Ben Shneiderman. "The eyes have it: A task by data type taxonomy for information visualizations". In: Proceedings 1996 IEEE symposium on visual languages. IEEE. 1996, pp. 336-343.

[207] Ben Shneiderman. "Tree visualization with tree-maps: 2d space-filling approach". In: ACM Transactions on graphics (TOG) 11.1 (1992), pp. 92-99. 
[208] Anna Sirkka, Johan Fagerlönn, Stefan Lindberg, and Katarina Delsing. "The design of an auditory alarm concept for a paper mill control room". In: Advances in Ergonomics In Design, Usability \& Special Populations: Part 3.18 (2014), p. 118.

[209] Anna Sirkka, Johan Fagerlönn, Stefan Lindberg, and Ronja Frimalm. "An auditory display to convey urgency information in industrial control rooms". In: International Conference on Engineering Psychology and Cognitive Ergonomics. Springer. 2014, pp. 533-544.

[210] Larisa Sitorus, Shan Shan Cao, and Jacob Buur. "Tangible user interfaces for configuration practices". In: Proceedings of the 1st international conference on Tangible and embedded interaction. ACM. 2007, pp. 223-230.

[211] Linda J Skitka, Kathleen L Mosier, and Mark Burdick. “Does automation bias decision-making?" In: International Journal of Human-Computer Studies 51.5 (1999), pp. 991-1006.

[212] Linda J Skitka, Kathleen L Mosier, Mark Burdick, and Bonnie Rosenblatt. "Automation bias and errors: are crews better than individuals?" In: The International journal of aviation psychology 10.1 (2000), pp. 85-97.

[213] Norman J Slamecka and Peter Graf. "The generation effect: Delineation of a phenomenon." In: Journal of experimental Psychology: Human learning and Memory 4.6 (1978), p. 592.

[214] Harvey S Smallman and Mark St John. "CHEX (Change History EXplicit): New HCI concepts for change awareness". In: Proceedings of the Human Factors and Ergonomics Society Annual Meeting. Vol. 47. 3. SAGE Publications Sage CA: Los Angeles, CA. 2003, pp. 528-532.

[215] Jonathan Smallwood and Jonathan W Schooler. "The restless mind." In: Psychological bulletin 132.6 (2006), p. 946. 
[216] Kip Smith and Peter A Hancock. "Situation awareness is adaptive, externally directed consciousness". In: Human factors 37.1 (1995), pp. 137-148.

[217] Justin Starren and Stephen B Johnson. "An object-oriented taxonomy of medical data presentations". In: Journal of the American Medical Informatics Association 7.1 (2000), pp. 1-20.

[218] Erik Stolterman. "The nature of design practice and implications for interaction design research". In: International Journal of Design 2.1 (2008).

[219] Jihoon Suh, Wooshik Kim, and Andrea Bianchi. "Button+: Supporting User and Context Aware Interaction through Shape-Changing Interfaces". In: Proceedings of the Eleventh International Conference on Tangible, Embedded, and Embodied Interaction. ACM. 2017, pp. 261-268.

[220] Don Tapscott. Grown up digital. McGraw-Hill Education Boston, 2008.

[221] Stuart Taylor, Cem Keskin, Otmar Hilliges, Shahram Izadi, and John Helmes. "Type-hover-swipe in 96 bytes: a motion sensing mechanical keyboard". In: Proceedings of the SIGCHI Conference on Human Factors in Computing Systems. ACM. 2014, pp. 1695-1704.

[222] Carlos E Tejada, Jess McIntosh, Klaes Alexander Bergen, Sebastian Boring, Daniel Ashbrook, and Asier Marzo. "EchoTube: Robust Touch Sensing along Flexible Tubes using Waveguided Ultrasound". In: Proceedings of the 2019 ACM International Conference on Interactive Surfaces and Spaces. ACM. 2019, pp. 147-155.

[223] Anand Tharanathan, Peter Bullemer, Jason Laberge, Dal Vernon Reising, and Richard Mclain. "Impact of functional and schematic overview displays on console operators' situation awareness". In: Journal of Cognitive Engineering and Decision Making 6.2 (2012), pp. 141-164. 
[224] James J Thomas and Kristin A Cook. "A visual analytics agenda". In: IEEE computer graphics and applications 26.1 (2006), pp. 10-13.

[225] John Tiab and Kasper Hornbæk. “Understanding Affordance, System State, and Feedback in Shape-Changing Buttons". In: Proceedings of the 2016 CHI Conference on Human Factors in Computing Systems. ACM. 2016, pp. 2752-2763.

[226] Marialena Vagia, Aksel A Transeth, and Sigurd A Fjerdingen. "A literature review on the levels of automation during the years. What are the different taxonomies that have been proposed?" In: Applied ergonomics 53 (2016), pp. 190-202.

[227] Alessandro Valli. "The design of natural interaction". In: Multimedia Tools and Applications 38.3 (2008), pp. 295-305.

[228] Elina Vartiainen, Veronika Domova, and Marcus Englund. "Expert on wheels: an approach to remote collaboration". In: Proceedings of the 3rd International Conference on Human-Agent Interaction. ACM. 2015, pp. 49-54.

[229] Roberto Verganti. Design driven innovation: changing the rules of competition by radically innovating what things mean. Harvard Business Press, 2009.

[230] Gianni Viano, Andrea Parodi, James Alty, Chris Khalil, Inaki Angulo, Daniele Biglino, Michel Crampes, Christophe Vaudry, Veronique Daurensan, and Philippe Lachaud. "Adaptive user interface for process control based on multiagent approach". In: Proceedings of the working conference on Advanced visual interfaces. ACM. 2000, pp. 201-204.

[231] Kim J Vicente, Emilie M Roth, and Randall J Mumaw. “How do operators monitor a complex, dynamic work domain? The impact of control room technology". In: International Journal of Human-Computer Studies 54.6 (2001), pp. 831-856. 
[232] Karel Vredenburg, Ji-Ye Mao, Paul W Smith, and Tom Carey. "A survey of user-centered design practice". In: Proceedings of the SIGCHI conference on Human factors in computing systems. 2002, pp. 471-478.

[233] JunPing Wang, WenSheng Zhang, YouKang Shi, ShiHui Duan, and Jin Liu. "Industrial Big Data Analytics: Challenges, Methodologies, and Applications". In: arXiv preprint arXiv:1807.01016 (2018).

[234] Yung-Sen Wang and Sheue-Ling Hwang. "An experimental study on the information design of CRT display in process control systems". In: International Journal of Industrial Ergonomics 15.6 (1995), pp. 459-469.

[235] Colin Ware. Information visualization: perception for design. Morgan Kaufmann, 2019.

[236] Susan Weinschenk. 100 things every designer needs to know about people. Pearson Education, 2011.

[237] Christopher Wickens. "Automation stages \& levels, 20 years after". In: Journal of Cognitive Engineering and Decision Making 12.1 (2018), pp. 35-41.

[238] Christopher D Wickens, Angelia Sebok, Huiyang Li, Nadine Sarter, and Andrew M Gacy. "Using modeling and simulation to predict operator performance and automationinduced complacency with robotic automation: a case study and empirical validation". In: Human factors 57.6 (2015), pp. 959-975.

[239] David D Woods. "Visual momentum: a concept to improve the cognitive coupling of person and computer". In: International Journal of Man-Machine Studies 21.3 (1984), pp. 229-244.

[240] Ming Xu, Hua Cai, and Sai Liang. "Big data and industrial ecology". In: Journal of Industrial Ecology 19.2 (2015), pp. 205210. 
[241] Holly A Yanco and Jill Drury. "“" Where am I?" Acquiring situation awareness using a remote robot platform". In: 2004 IEEE International Conference on Systems, Man and Cybernetics (IEEE Cat. No. 04CH37583). Vol. 3. IEEE. 2004, pp. 2835-2840.

[242] Xing-Dong Yang, Edward Mak, David McCallum, Pourang Irani, Xiang Cao, and Shahram Izadi. "LensMouse: augmenting the mouse with an interactive touch display". In: Proceedings of the SIGCHI Conference on Human Factors in Computing Systems. ACM. 2010, pp. 2431-2440.

[243] Robert M Yerkes and John D Dodson. "The relation of strength of stimulus to rapidity of habit-formation". In: Journal of comparative neurology and psychology 18.5 (1908), pp. 459-482.

[244] Robert B Zajonc. "Social facilitation". In: Science 149.3681 (1965), pp. 269-274.

[245] Haimo Zhang and Yang Li. "GestKeyboard: enabling gesture-based interaction on ordinary physical keyboard". In: Proceedings of the SIGCHI Conference on Human Factors in Computing Systems. ACM. 2014, pp. 1675-1684.

[246] John Zimmerman, Shelley Evenson, and Jodi Forlizzi. “Discovering and Extracting Knowledge in the Design Project". In: (2004).

[247] John Zimmerman and Jodi Forlizzi. "Research through design in HCI". In: Ways of Knowing in HCI. Springer, 2014, pp. 167-189.

[248] John Zimmerman and Jodi Forlizzi. "The role of design artifacts in design theory construction". In: Artifact: Journal of Design Practice 2.1 (2008), pp. 41-45.

[249] John Zimmerman, Jodi Forlizzi, and Shelley Evenson. "Research through design as a method for interaction design research in $\mathrm{HCI}^{\prime \prime}$. In: Proceedings of the SIGCHI conference on Human factors in computing systems. ACM. 2007, pp. 493-502. 
[250] Goranka Zoric, Veronika Domova, Maria Ralph, Elina Vartiainen, Petra Björndal, and Alvaro Aranda Muñoz. "Supporting maritime remote experts working over distance". In: Proceedings of the 9th Nordic Conference on Human-Computer Interaction. ACM. 2016, p. 124.

[251] Shoshana Zuboff. "In the age of the smart machine. The future of power and work". In: New York: Basic (1988). 


\section{Papers}

The papers associated with this thesis have been removed for copyright reasons. For more details about these see:

http://urn.kb.se/resolve?urn=urn:nbn:se:liu:diva-165625 


\section{FACULTY OF SCIENCE AND ENGINEERING}

Linköping Studies in Science and Technology Dissertation No. 2077, 2020

Division of Media and Information Technology

Linköping University

60174 Norrköping, Sweden

www.liu.se 